\title{
Linx
}

Revue des linguistes de l'université Paris X Nanterre

$78 \mid 2019$

La linguistique des genres, en actes et en questions

\section{Linguistique des genres : objet et méthode}

Statut culturel des genres et variétés génériques

Genre in linguistics : epistemological and methodological principles. Cultural

Status of Genres and description of Genres Varieties

\section{Christophe Gérard}

\section{OpenEdition}

Journals

Édition électronique

URL : https://journals.openedition.org/linx/3030

DOI : 10.4000/linx.3030

ISSN : 2118-9692

Éditeur

Presses universitaires de Paris Nanterre

Référence électronique

Christophe Gérard, «Linguistique des genres : objet et méthode », Linx [En ligne], 78 | 2019, mis en ligne le 30 juin 2019, consulté le 26 juillet 2022. URL : http://journals.openedition.org/linx/3030 ; DOI : https://doi.org/10.4000/linx.3030

Ce document a été généré automatiquement le 26 juillet 2022.

Tous droits réservés 


\title{
Linguistique des genres : objet et méthode
}

\author{
Statut culturel des genres et variétés génériques ${ }^{1}$ \\ Genre in linguistics : epistemological and methodological principles. Cultural \\ Status of Genres and description of Genres Varieties
}

\section{Christophe Gérard}

Ignorer la nature de l'énoncé et les particularités de genre qui marquent la variété du discours dans un quelconque domaine de l'étude

linguistique mène au formalisme et à l'abstraction, dénature l'historicité d'une étude, affaiblit le lien qui existe entre la langue et la vie.

(Bakhtine 1984 : 268).

[...] l'état historique d'un genre ne constitue pas une entité ou structure systématique, mais plutôt

une multiplicité ou architecture de systèmes, comparable, là encore, à l'état d'une langue [...]. Ce diasystème peut être structuré du point de vue spatial (diatopique), socioculturel (diastrastique) et stylistique (diaphasique). (Kuon 2015 : 187).

1 Malgré le savoir élaboré depuis les années 1950, la linguistique des genres ne répond pas encore aux exigences de ses nombreux enjeux scientifiques (Günthner/Knoblauch 1995 : 1-4), qui s'étendent de l'histoire de la langue ${ }^{2}$ à la description des conduites humaines (usages et coutumes) ${ }^{3}$, en passant par la traduction et l'acquisition du langage 4 . En effet, ce champ de recherches, qui demeure aujourd'hui au mieux considéré comme une « linguistique parallèle » (Adamzik 2018a: 3$)^{5}$, se signale par une profonde fragilité dont les symptômes reflètent les problèmes fondamentaux des sciences du langage actuelles: sa parcellisation ${ }^{6}$, son hyperspécialisation terminologique ${ }^{7}$ et sa volatilité épistémologique ${ }^{8}$ causant inévitablement une érosion du savoir commun indispensable au fonctionnement de la science. 
2 Devant ces difficultés, la linguistique des genres ne peut espérer progresser ni par la quête illusoire d'un modèle théorique unique, ni par la sélection dogmatique d'une conception ou d'une théorie particulière ${ }^{9}$, ni bien entendu en continuant d'accumuler des descriptions où, trop souvent, la notion de " genre » reste un mot-clé que les études ne prennent pas la peine de définir ${ }^{10}$. Face à cette situation, où la réflexion épistémologique devrait être considérée comme prioritaire, cet article entend contribuer à la discussion, voire susciter le débat, en apportant des éléments de réponse aux questions suivantes :

3 (a) Malgré la forte hétérogénéité de ce champ de recherches, ne peut-on s'accorder sur un agencement interne primaire, c'est-à-dire sur les principales lignes de partage de ce champ et sur sa cohérence de fond (infra 2 et 3), qui permette de rompre avec l'isolationnisme des théories partielles, dont certaines sont assurément complémentaires, comme on peut le voir à propos de la relation texte-genre (ex. infra 1.2)?

4 (b) Malgré les constats consternés et pessimistes (Adamzik 2001:21), ne peut-on néanmoins rectifier, dans ce domaine, une terminologie devenue opaque et plurivoque ${ }^{11}$, tout en acceptant par ailleurs l'« unification terminologique impossible » de la linguistique (Neveu 2008 : 92), voire celle de la linguistique textuelle (Adamzik 2018a : 4), branche mère de la linguistique des genres ?

5 (c) En particulier, dans la mesure où les genres sont des objets culturels (infra 4), ne peut-on commencer par reconsidérer les catégories descriptives de tradition diomédienne (i.e. relevant d'une classification organisée en "genera » et «species ») ? Comment considérer les critiques adressées aux catégories de la "classe ${ }^{12}$ et du "sous-genre " ${ }^{13}$ (et à son (b) homologue récent "hypergenre»), qui continuent pourtant de déterminer la conception habituelle de l'espace des normes médiatisant la langue et le texte (infra 1.1) ?

6 (d) La focalisation exclusive sur la notion de genre n'est-elle pas excessive? $\mathrm{Ne}$ détourne-t-elle pas notre attention de phénomènes sociodiscursifs apparentés (comme les styles collectifs, infra 1.1) qui, eux aussi, conditionnent la production et la réception des textes (oraux et écrits), et plus généralement les échanges communicationnels au sein d'une culture?

7 (e) Au-delà, et plus essentiellement, l'approche linguistique des genres ne doit-elle pas se défaire d'un certain logocentrisme qui l'empêche de reconnaître pleinement le statut culturel des genres (infra 4) - et qui fait obstacle à la saisie d'observables rarement mis en avant, comme la variation générique (infra 5) ? En définitive, n'est-il pas temps pour la linguistique des genres de se repenser à partir du lien entre langage et culture (Sherzer 2012), pour développer un point de vue supérieur, plus global et ethnologisé, qui aurait pour effet de définir les genres, d'emblée et de la manière la plus maximale, comme des manifestations historiques de la diversité communicationnelle propre à chaque culture?

8 En réponse à ces interrogations, nous commencerons par délimiter le champ de la linguistique des genres, en situant les genres parmi les niveaux du langage (1), puis en exposant son objet idéal et certains critères méthodologiques fondamentaux (2). La troisième section tracera les grandes lignes de partage qui traversent ce champ d'études, au-delà des multiples approches divergentes qui le composent (3). On développera ensuite une conception ethnologique des genres qui nous conduira à poser le principe que l'usage culturel d'un genre n'est pas nécessairement uniforme et qu'il 
convient, par conséquent, d'intégrer la notion de variété à la problématique des genres (4). Enfin, les deux dernières sections ont pour but de systématiser le phénomène de la variation générique en dotant le linguiste et l'ethnologue de nouveaux concepts descriptifs (5 et 6 ).

L'objectif principal de cet article est ainsi de réaffirmer le lien indissoluble entre histoire, culture et genres, en montrant la nécessité de développer une conception variationniste en linguistique des genres (Adamzik 2008), dont la théorie et la pratique suivent les principes des sciences de la culture (Rastier 2001b, Fix 2011). En effet, si l'on considère, contrairement aux approches structuralistes et formalistes (Glessgen 2018 : 14-15), que la variation n'est pas un épiphénomène, mais qu'elle est un des aspects constitutifs du langage, alors il n'y a aucune raison de limiter l'étude de la variation aux langues/dialectes, c'est-à-dire aucune raison de ne pas l'étendre aux genres discursifs, et plus généralement aux traditions discursives. Et, conséquemment, on devrait reconsidérer la variation linguistique comme étant composée d'un côté, traditionnellement, du domaine de la variation idiomatique, et de l'autre du domaine peu exploré de la variation discursive, dont la variation générique est une partie. On conforme ainsi le point de vue linguistique à l'impératif variationnel des cultures.

\section{Situation des genres parmi les niveaux du langage}

\subsection{Genres, espace des normes et traditions discursives}

Bien que l'expression "linguistique des genres" soit très rarement employée en linguistique de langue française ${ }^{14}$, et qu'elle ne désigne par conséquent pas un champ de recherches explicitement identifié comme tel par la communauté des linguistes, on est toutefois en droit d'y faire correspondre l'ensemble des travaux qui, des années 50 à nos jours (Bouquet $2004: 4-7$ ), se sont consacrés aux "genres du discours », " genres discursifs », " genres de textes ", " genres textuels ", " genres de la parole», ou encore aux « types de texte » et aux « types de discours », voire aux « styles de texte ».

11 Malgré leur disparate, ces travaux explorent une même réalité qualifiable de discursive, c'est-à-dire une réalité langagière distincte, d'un côté, de la langue et de ses variétés, et de l'autre de l'ensemble des textes (oraux et écrits) singuliers (passés, présents et à venir). Plus précisément, selon une conception qui s'inscrit dans le legs de Bakhtine ${ }^{15}$, l'objet de la linguistique des genres se situe au niveau du " "chaînon manquant" entre la langue et la parole [...] constitué par l'espace des normes ${ }^{16}$ dans lequel entre l'enfant au cours de sa socialisation progressive »(Rastier $2007: 4$, je souligne).

12 Alors que la complexité interne de cet espace reste à explorer - chaque communauté culturelle y organise la pluralité de ses genres en « champs » et en " réseaux » (Hauser 2014), et même en " chaînes » (Adamzik 2001), on peut a minima le situer globalement parmi les différents niveaux du langage. Dans cette optique, l'espace des normes relève du niveau historique du langage, un niveau qui comprend certes les genres, mais aussi d'autres types de traditions discursives ${ }^{17}$. Ce dernier terme a en effet été initialement forgé par P. Koch (1997 : 43-45) dans le but de compléter la conception cosérienne des trois niveaux du langage ${ }^{18}$ :

[...] c'est d'abord l'historicité des langues particulières et de leurs variétés qui saute aux yeux. Cependant, il s'est avéré indispensable d'y opposer, en précisant l'approche de Coseriu, un second type de l'historicité du langage qui comporte 
notre capacité de produire des discours/des textes selon des traditions et des modèles historiques, logiquement indépendants des langues historiques particulières. Force est donc de diviser le niveau historique [...] en deux domaines, à savoir celui des langues (particulières) et celui des traditions discursives. (Koch 2015b : 79).

13 En reconnaissant ainsi que l'activité de parler est régie par des règles/normes discursives qu'il convient de bien distinguer des règles/normes idiomatiques (i.e. de langue), Koch rendait le modèle de Coseriu plus conforme à la réalité langagière ${ }^{19}$ :

\begin{tabular}{|l|l|l|}
\hline Niveau & \multicolumn{2}{|l|}{ aspect du langage } \\
\hline universel & activité de parler \\
\hline historique & langues/variétés & traditions discursives \\
\hline actuel & \multicolumn{2}{|l|}{ discours [texte] } \\
\hline
\end{tabular}

Figure 1 : les niveaux du langage selon P. Koch.

La notion de tradition discursive est particulièrement éclairante pour délimiter le champ de la linguistique des genres, car elle met en lumière le fait que l'espace des normes est certes occupé par des genres, mais aussi que ces derniers interagissent avec d'autres traditions discursives, qui elles aussi sont également impliquées dans l'orchestration sociale de la communication ${ }^{20}$ :

La notion de 'tradition discursive' inclut, entre autres, celle de 'genre littéraire', mais elle va bien au-delà de celle-ci, passant à travers la grande variété des univers $\mathrm{du}$ discours (littérature, histoire, droit, religion, science, etc.). Voici, en vrac, quelques exemples de traditions discursives : style sublime, atticisme, maniérisme ; article de fond, sonnet, causerie, blague, chat; actes de langage du baptême, acte de langage du serment; formules de salutation, formule de remerciement, formule de pénalité, etc. (Koch 2015b : 79).

De fait, si « tous les genres sont des traditions discursives, les traditions discursives, par contre, ne sont pas nécessairement des genres" (Kabatek 2015a: 204). Plus précisément, outre les genres proprement dits («sonnet, causerie, blague », etc.), et outre «les formules de salutation, formule de remerciement, formule de pénalité, etc. ", qui renvoient à la catégorie de la forme discursive (Wilhelm 2001, $2003^{21}$ ), Koch mentionne ici une autre catégorie de normes qu'on peut appeler style collectif (par opposition au style individuel, aussi nommé style d'auteur $)^{22}$ : "style sublime, atticisme, maniérisme", auxquels on peut ajouter les registres cicéroniens ${ }^{23}$, le burlesque français du XVII ${ }^{\mathrm{e}}$ siècle ${ }^{24}$, le baroquisme, le symbolisme, le surréalisme, etc. Il apparaît donc qu'au sein de l'espace des normes le genre ne peut être conçu comme une catégorie isolée, existante et agissante seule, et par conséquent qu'une linguistique des genres non restreinte devrait compter parmi ses thèmes d'étude les rapports entre genre et forme discursive d'un côté25, et de l'autre les rapports entre genre et style collectif.

Concernant ce second thème de recherche en particulier, on notera tout d'abord que, contrairement à ce que laissent penser les exemples ci-dessus, la catégorie du style collectif ne concerne pas seulement le domaine de la littérature. En effet, dans le domaine de la presse par exemple, la ligne éditoriale d'un journal définit un style collectif particulier (soit, dans ce cas, un ton éditorial) qui se manifeste par des 
différences d'usage lexical et syntaxique patentes entre quotidiens, notamment concernant la création lexicale (Ollinger/Valette 2010, Gérard 201826). Plus généralement, l'anthropologie linguistique a montré que, au-delà des genres, les styles collectifs ont pour fonction, au sein d'une culture, de définir l'identité de certains groupes sociaux (Irvine 2001). Mais, surtout, pour notre propos, on retiendra que le style collectif interagit avec le genre en déterminant l'usage de ce dernier au sein d'une communauté : on assiste alors, pour un même genre, à des variations parfois très fortes, comme l'illustre en France la pratique de l'éditorial dans les journaux Le Monde et Libération ${ }^{27}$, ou la forte présence de régionalismes dans l'horoscope du site alsacien www.madeinalsace.com. De semblables cas montrent que le linguiste ne peut se fier au seul principe que « tout texte relève d'un genre » (infra 1.2), sans examiner si et en quoi les normes de ce dernier sont adaptées sous l'influence d'un style collectif plus global. Par conséquent, pour s'ajuster à la complexité des phénomènes discursifs, la linguistique des genres devrait reformuler le précédent principe («tout texte relève d'un genre ") en posant, d'une manière moins restrictive, que tout texte implique potentiellement un rattachement multiple à différentes traditions discursives, dont les genres font partie.

Pour finir ici sur les difficultés terminologiques évoquées en introduction, on notera que l'emploi de la notion de tradition discursive peut être un moyen de faire face aux confusions terminologiques touchant la notion de genre (désignations multiples, définitions diverses). De fait, le terme plus large de tradition discursive peut utilement être substitué à celui de genre pour désigner, globalement, l'action de normes discursives sur la libre expression des individus (infra 1.2), sans préciser s'il s'agit de genre, de style collectif ou d'une autre catégorie. Et, de fait, les genres de la déclaration d'impôts française, de l'interview journalistique, du CV anglais, du roman sentimental, etc. ne sont autres que, d'une manière primaire, des traditions discursives; éviter de les nommer "genre» permet d'esquiver les complications terminologiques habituelles. Mieux, en ce sens, au lieu de viser une linguistique des genres, il semble plus ambitieux et plus pertinent d'aspirer à une linguistique des traditions discursives, qui a pour intérêt de poser d'emblée la nature historico-culturelle des genres (infra 4), sans oublier leur interaction avec d'autres catégories de traditions discursives (dont la typologie reste à fixer, Kabatek 2015b) situées au même niveau historique du langage.

\subsection{Complexité de la relation entre genre et texte}

D'un point de vue théorique, les genres sont généralement conçus comme des modèles communicationnels qui contraignent invariablement, de multiples manières, la libre expression verbale des individus ${ }^{28}$. Comme le soulignait déjà Bakhtine :

Le vouloir dire du locuteur se réalise avant tout dans le choix d'un genre du discours. [...] le dessein discursif du locuteur, sans que celui-ci se départisse de son individualité et de sa subjectivité, s'adapte et s'ajuste au genre choisi, se compose et se développe dans la forme du genre donné. (Bakhtine 1984 : 284).

19 Ce principe général est aujourd'hui admis comme un savoir acquis, différemment formulé dans les traditions scientifiques de langue française (dont Adam, Rastier ${ }^{29}$ ), anglaise (e.g. Biber 1988, Lee 2001), allemande (e.g. Koch 1997, Brinker 2001), etc. Cela étant, la relation entre un texte individuel et « son » genre est loin d'aller de soi et il convient d'en souligner toute la complexité, contre de trop fréquentes simplifications, et à l'encontre de la « rigidité » que certains croient voir en la notion de genre : 
1) l'appartenance d'un texte à un genre peut être plus ou moins forte (ou " graduelle », Adam 2012 : 19), différents facteurs pouvant faire varier le degré de conformité générique d'un texte à son modèle ${ }^{30}$;

2) qu'un texte relève d'un genre ne l'empêche pas d'en inclure d'autres, ces genres inclus (Rastier 2001a: 266) pouvant être permis (Freyermuth 2011) ou bien prescrits par le genre incluant (ex. la « lettre» dans le roman épistolaire) ;

3) un texte peut ne pas relever d'un genre unique (Adamzik 2004 : 101-102), en particulier en littérature dans les cas d'«hybridation générique " (Fix 1997, Heidmann/Adam 2010, Adam 2012), le processus d'hybridation pouvant d'ailleurs être à l'origine de nouveaux genres (Koch 1997);

4) la relation entre un texte et "son " genre peut connaître une réactualisation réceptive (Schaeffer 1989: 141-155; aussi Adam 2012: 17-19), c'est-à-dire une recatégorisation générique due à un changement de contexte historique; ce contexte incluant l'épistémè en vigueur au moment de la réception (par ex. le genre de l'epyllion hellénistique ne (re)commence à exister qu'à la fin du XIX ${ }^{\mathrm{e}}$ siècle, en tant que reconstitution philologique (Cusset 2016)).

5) on ne peut ignorer que certains textes marginaux doivent être considérés comme a-génériques, telle une partie des «écrits bruts» (provenant des hôpitaux psychiatriques), qui sont par définition des actes de non-communication. Plus généralement, il est improbable que la diversité des genres puisse recouvrir, sans aucun reste, la totalité des textes individuels existants et à venir.

6) Enfin, on l'a vu, un texte peut renvoyer en même temps à d'autres types de traditions discursives distincts des genres (supra 1.1), comme les styles collectifs qui peuvent en particulier déterminer l'usage d'un genre ou d'un groupe de genres.

Toutes ces raisons appellent à relativiser, au bénéfice d'une théorisation plus exacte, le principe d'appartenance d'un texte à un genre. À ce point de l'exposé, les développements précédents n'ont fait que situer l'objet la linguistique des genres parmi les autres faits de langage, au sein de l'espace des normes. Exposons à présent ses dimensions définitoires.

\section{L'objet et la méthode de la linguistique des genres}

\subsection{Le genre : un objet tridimensionnel}

21 À un certain niveau d'abstraction, indépendant de celui des approches et des théories linguistiques particulières (infra 3), l'« objet idéal » de la linguistique des genres peut être délimité, ou tout du moins précisé, en prenant pour motif heuristique l'analogie entre les langues et les genres que Coseriu formule au début des années $1970^{31}$ :

Dans les activités humaines, nous constatons une combinaison, une dialectique entre transmissions, traditions historiques et création individuelle. Quand ces traditions représentent des structures générales, elles s'appellent "genres » en poésie et « langues » dans la langue.

Ce parallélisme entre les genres littéraires et les langues nous paraît fondamental, aussi bien pour la théorie des genres que pour la théorie de la langue. (Coseriu $1976: 20$, cité et traduit par Kuon $2015: 177)$.

En étendant cette analogie au-delà du domaine littéraire, c'est-à-dire en considérant exclusivement la nature historique commune à ces deux objets culturels (les langues et les genres en général), la linguistique des genres semble disposer d'un mode de questionnement fécond, permettant de la rapprocher d'« une théorie des genres qui soit cohérente aussi bien du point de vue théorique, que du point de vue méthodologique. » (Kuon 2015 : 178). 
23 À la suite de Kuon, ce questionnement par analogie nous conduit à la nécessité de délimiter l'objet de la linguistique des genres au moyen de trois dimensions: la conception, la fonction et l'évolution. Ces dimensions, définitoires du genre en tant qu'objet linguistique (i.e. étudié seulement dans sa dimension verbale), proviennent d'une réflexion d'Oesterreicher $(1979: 279)$ que Kuon applique ainsi à la problématique des genres :

Le point de vue de la conception a pour objet la distinction des niveaux articulatoires des genres (notamment les formes de présentation, les modalités du langage, la rhétorique du texte, les motifs, les sujets, les unités de sens micro-structurelles simples et macro-structurelles complexes, etc.), l'analyse de la production du sens propre au genre (comme le problème de la systématicité ainsi que les éléments paradigmatiques et syntagmatiques entre les éléments du texte) et le déploiement de la variété diasystématique d'un genre. Le point de vue de la fonction s'intéresse avant tout aux aspects pragmatiques de la situation de communication, le rapport d'un genre avec la réalité et son insertion dans la société. En ce qui concerne enfin l'évolution, il faut une fois de plus rappeler la définition du genre présentée cidessus: si l'on comprend les genres comme des objets idéaux culturels dont les changements sont dus à des choix créateurs qui, bien que libres, sont faits dans des conditions historiques (externes ou internes) bien déterminées, alors il faudra rejeter comme inappropriées toutes les approches [...] qui cherchent à réduire le changement des genres à des régularités causales (Kuon 2015 : 184).

Cette caractérisation tridimensionnelle dessine l'objet idéal de la linguistique des genres, conçu dans son intégralité, dans la mesure où il ne semble pas qu'on puisse réduire ou augmenter le nombre de ces trois dimensions. Si bien que la négligence d'une d'entre elles appelle une critique justifiée, comme ce fut le cas pour l'approche structurale des genres qui s'est vue reprocher, par les approches pragmatiques (sur la visée illocutoire des genres, e.g. Schaeffer 1989: 101-14), sa méconnaissance de la dimension fonctionnelle. Ainsi, alors que la déposition judiciaire et le témoignage littéraire (des génocides, en particulier ${ }^{32}$ ) produisent tous deux des récits de vie, ces derniers restent nettement caractérisés par des motifs et des intentions bien spécifiques.

Sous un angle positif toutefois, il faut en même temps souligner que la focalisation sur une de ses trois dimensions peut tout aussi bien procéder d'une visée délibérée, et par là méthodologiquement légitim(é)e. C'est le cas pour la linguistique historique des genres (infra 2.4), qui se concentre sur l'évolution (par ex. celle du polar régional français depuis une quinzaine d'années, celle du battle rap américain entre 1980 et $2000^{33}$, celle de l'essai français au XX $\mathrm{XX}^{\mathrm{e}}$ siècle (Macé 2006), ou celle des messages codés de la Résistance entre 1940 et 1944). De même, il est licite d'opérer la caractérisation linguistique d'un genre donné du seul point de vue de la conception, en excluant explicitement, par méthode, les autres dimensions. Dès lors, sans préciser les spécificités fonctionnelle et historique du genre en question, on ne décrit que les règles/normes qui correspondent à un usage particulier de la langue et à une textualité particulière, comme dans la représentation suivante ${ }^{34}$ du genre du slam, limitée à ses normes verbales ${ }^{35}$ :

\begin{tabular}{|l|l|l|l|}
\hline & Obligations & Interdictions & Permissions \\
\hline \multirow{2}{*}{ Langue } & $\begin{array}{l}\text { «métissage langagier } \\
\text { modalisation marquée } \\
\text { variation diamésique }\end{array}$ & registre vulgaire ${ }^{37}$ & $\begin{array}{l}\text { variation diatopique } \\
\text { variation diachronique } \\
\text { technolecte }\end{array}$ \\
\hline
\end{tabular}




\begin{tabular}{|l|l|l|l|}
\hline \multirow{2}{*}{ Textualité } & rythme et rimes & texte long & mention du titre \\
& figures de style & parole improvisée & type compositionnel libre \\
thèmes urbains/actuels & argumentum ad personam & hypertextualité (Genette) \\
\hline
\end{tabular}

Figure 2 : caractérisation linguistique du genre du slam.

Et d'une manière plus restreinte encore, il est possible de ne se concentrer que sur la dimension sémantique de la textualité, comme le fait Rastier (1989 et 2001a), sans décrire les caractéristiques du genre qui concernent l'usage de la langue (i.e. de ses variétés lexicales, ses relations syntaxiques, etc.), c'est-à-dire en omettant la dimension $\mathrm{du}$ « registre » au sens de Biber/Conrad (2009, infra 3.2).

\subsection{Genres historiques et genres théoriques/de méthode}

Cependant, la définition totale de son «objet idéal» (tridimensionnel) devrait normalement guider la linguistique des genres, et même toute description linguistique faisant appel à ces derniers, d'une manière ou d'une autre. À cet égard, outre l'importance de la dimension fonctionnelle, notamment pour interpréter les caractéristiques linguistiques d'un genre (ex. Biber/Conrad 2009: 6-8), il convient tout spécialement de ne pas négliger le point de vue de l'évolution historique ${ }^{38}$, car c'est précisément cette négligence qui conduit la plupart du temps à des méthodologies approximatives.

En effet, reconnaître l'historicité des genres ou, ce qui revient au même, poser qu'un genre se définit comme un modèle doté d'une histoire propre (ancienne ou récente, en cours ou révolue), c'est exiger d'emblée de sa propre méthodologie qu'elle se positionne par rapport à une définition empirique du genre (supra 1.1, infra 4), et qu'elle clarifie de ce point de vue la nature de son matériau générique (i.e. de son corpus), quitte à poser explicitement que ce dernier relève d'une construction théorique ou d'une décision de méthode.

À ce titre, on sait que c'est à partir de la Renaissance que l'épopée, la tragédie et la comédie acquièrent leur valeur de "genres canoniques" (Schaeffer $1989: 28$ ), et que le romantisme allemand consacre, lui, la triade épopée/lyrisme/drame (Kuon 2015 : 177). Mais de telles catégories génériques, qui sont respectivement le fruit de la pensée d'Aristote et de Hegel, posent la question, essentielle du point de vue méthodologique, de savoir si la description linguistique se fonde sur d'authentiques genres historiques ou, au contraire, sur ce que Todorov (1970) a nommé des " genres théoriques » - «tels qu'ils sont définis par tel ou tel critique [ou par tel ou tel linguiste, CG] » (Schaeffer 1989: $\left.68^{39}\right)$, ou encore sur ce que nous pourrions appeler des "genres de méthode " pouvant notamment servir, de manière ad hoc, à organiser les parties d'un corpus ${ }^{40}$.

Recourir à des genres théoriques ou à des genres de méthode est en soi parfaitement légitime, quand leur usage fait sens au sein d'un projet intellectuel qui confère à ces sortes de genre une portée conceptuelle ou une légitimité méthodologique (Kabatek 2015a : 200). Mais le fait que tel critique ou tel linguiste puisse à bon droit employer ces catégories de connaissance ne signifie pas que le recours aux genres historiques soit une possibilité méthodologique parmi d'autres: les genres historiques sont au contraire 
essentiels parce qu'ils sont les seuls à pouvoir satisfaire l'empirisme qui définit l'activité descriptive en linguistique.

\subsection{Dimension historique des genres et champs génériques : méthodologie critique}

31 Tout d'abord, reconnaître cette dimension historique revient à poser un critère qui devrait conduire à refuser la qualification de genre aux catégories textuelles dénuées de cette dimension, comme c'est le cas, dans la citation suivante, de la «description », la "polémique» et le «récit», à la différence du «roman sentimental», de la « tragédie », du « sonnet », du " reality-show », de la « carte de vœux », etc. :

Les locuteurs disposent d'une foule de termes pour catégoriser l'immense variété des textes qui sont produits dans une société : 'conversation', 'manuel', 'journal', 'tragédie', 'reality-show', 'roman sentimental', 'description', 'polémique', 'sonnet', 'récit', 'maxime', 'hebdo', 'tract', 'rapport de stage', 'mythe', 'carte de vœux'... On notera que la dénomination de ces genres s'appuie sur des critères très hétérogènes. (Maingueneau $1998: 45$ ).

Rappelons d'ailleurs que la "description » et les catégories apparentées (" narration », « argumentation », « dialogue », etc. $\left.{ }^{41}\right)$ ne peuvent tout simplement pas être qualifiées de genre puisque, précisément, c'est ce dernier qui en détermine la présence dans les textes, la qualité spécifique et la fonctionnalité, non seulement en discours, occasionnellement, mais aussi et surtout au niveau des traditions d'une communauté linguistique donnée :

Les séquences ne sont pas les mêmes selon les genres et les champs génériques : la description romanesque diffère de la description poétique ; en poésie, la description dans la satire ou dans l'élégie n'ont presque rien de commun. [...]. En effet, leurs usages eux-mêmes dépendent du genre, et ne le définissent pas : par exemple, le dialogue se trouve dans la poésie parodique ou légère, mais non dans la poésie lyrique. (Rastier 2001a : 265).

Cependant, reconnaître l'historicité d'une catégorie représentant un certain degré de généricité, comme ici «la tragédie» (ou encore «la comédie»), ne suffit pas nécessairement pour identifier un genre historique stricto sensu. Ainsi, alors qu'on ne peut dénier l'historicité de ce qu'on appelle communément "le roman», ce dernier n'est pas pour autant assimilable à un genre historique : à proprement parler, tout comme «le manga » (infra 4.4), «le roman » a le statut d'une abstraction subsumant une grande diversité de genres qui, eux, ont chacun bien connu une évolution propre, constituant chacun une "lignée générique " (Rastier 2001a : 252) - comme le roman libertin (de Crébillon fils à Bataille, etc.), le roman sentimental (de Delly et Cartland à Marc Levy, etc.), le roman historique (de Walter Scott à Ken Follett), le roman d'énigme (de Christie à Simenon, etc.), etc. Autre exemple, Biber et Conrad (2009: 19-21) considèrent "la poésie ", "le drame» (i.e. le texte théâtral) et la "prose fictionnelle » comme autant de genres littéraires («three major literary genres»). Mais la "prose fictionnelle " est une méta-catégorie qui ne possède aucune historicité propre et les deux autres catégories génériques sont disjointes des filiations historiques qu'elles résument. De fait, les genres poétiques proprement dits sont avant tout ceux qui ont été reconnus par la tradition et qui ont été transmis entre poètes (idylle, élégie, ode, sonnet, épigramme, rondeau, madrigal, etc. ${ }^{42}$ ). 

souvent des classifications analogues, comme la dernière version en date de Frantext (www.frantext.fr) qui certes qualifie justement de «genre textuel » les mémoires, l'essai et le récit de voyage, mais qui retient aussi la " prose » (?), le « roman », la " poésie » et le "théâtre ", sans distinguer pour ce dernier les genres historiques du mystère, de la tragi-comédie, du mélodrame, de la comédie satirique, du vaudeville, du drame bourgeois, etc. Toutefois, malgré son échelle de généricité hétérogène, Frantext permet encore d'envisager des descriptions sociodiscursivement pertinentes, à la différence d'une base de données comme Sketch Engine (www.sketchengine.eu) dont les soi-disant genres («news », « general », « business », « medical», « fiction», etc.) restent tout à fait inexploitables de ce point de vue. pertinentes - c'est-à-dire les plus adéquates à l'objet idéal d'une linguistique des genres (supra 2.1) - sont en toute rigueur celles qui identifient des « genres historiques au sens le plus fort du terme» (Schaeffer 1989: 117 et infra 2.4), comme la tragédie élisabéthaine, la tragédie classique, le roman antique, le sonnet baroque, etc. Ces dénominations expriment une conscience aigüe de l'historicité des genres (et de leur identité culturelle, infra 4 et 5) qui, au fond, impose à la méthode d'analyse l'exigence d'un réalisme historique (dont Sketch Engine est totalement dépourvu). Et à cet égard, il faut bien reconnaitre que la liste des "genres seconds du discours " que donne Bakhtine est loin d'être exemplaire : «le roman, le théâtre, le discours scientifique, le discours idéologique, etc. » (Bakhtine $1984:$ 267). Au-delà, la volonté de formuler des dénominations de genre précises, qui est un acte critique vis-à-vis des dénominations trop généralisantes («le roman", mais aussi "le débat", "la conversation", «la conférence ", etc.) et des allégations de « protypicité » de tel ou tel genre, traduit une conscience des limites d'une linguistique textuelle trop "systémique ", qui doit encore tirer les leçons de la linguistique variationnelle (Adamzik 2008, infra 5). des genres historiques à proprement parler, mais si ces dénominations renvoient à des familles de (véritables) genres? Il est pertinent et opératoire d'y voir des champs génériques $^{44}$, en employant un concept qui toutefois reste à interroger (Koch 2015b) ${ }^{45}$. On parlera ainsi du champ générique du récit romanesque au lieu «du roman », du champ générique de la poésie au lieu de "la poésie », etc. À cet égard, la typologie des champs lexicaux de Coseriu (2001: 405) se révèle heuristiquement utile pour concevoir différents types de champs génériques selon leur l'organisation interne spécifique. Dans cette perspective, le champ générique de l'article de presse serait qualifiable de bidimensionnel, car classiquement divisé en genres d'opinion (éditorial, critique, tribune libre, etc.) et genres d'information (reportage, enquête, portrait, etc.) (Grosse 2001). Quant au champ du théâtre (occidental contemporain), il serait qualifiable de tri-dimensionnel au sens où l'activité théâtrale a fini par élaborer une organisation ternaire (comédie/ tragédie/drame).

Toutes les considérations précédentes, qui ont porté sur la dimension historique des genres, ne forment pourtant qu'une partie d'une réflexion qui doit encore être prolongée. Car cette dimension historique ne doit pas masquer la dimension culturelle des genres, soulignée par différents linguistes (appartenant à différentes traditions scientifiques $\left.{ }^{46}\right)$, mais que met plus clairement en avant l'ethnologie contemporaine : 
In accordance with Hanks (1987), we argue that communicative genres can be treated as historically and culturally specific conventions and ideals according to which speakers compose talk and recipients interpret it (Günthner/Knoblauch 1995 :20).

Cet accent ethnologique mis sur les spécificités culturelles des genres (infra 4), qui reste sous-exploité en linguistique textuelle, vient alors compléter une méthodologie critique qui, quand elle écarte la voie des genres théoriques ou des genres de méthode, cherche à se tenir au plus près des réalités historico-culturelles.

\subsection{Distinctions méthodologiques fondamentales et hypothèse de la variation générique}

Le parallèle entre les langues et les genres, commencé plus haut avec Kuon (supra 2.1), mérite d'être poursuivi sur le plan des distinctions fondatrices de l'étude moderne du langage (synchronie, diachronie, etc.). En effet, celles qui ne s'appliquent pas spécifiquement aux langues dotent, en partie, la description des genres de la rigueur méthodologique requise dans ce domaine - en amont et indépendamment du cadre théorique adopté pour rendre compte des observables visés sur un corpus donné (infra 3). À cet égard, la description linguistique des genres peut par exemple expliciter sa méthodologie en s'inspirant des niveaux d'analyse classiques, comme le fait Schaeffer en détaillant les niveaux énonciatif, sémantique et syntaxique "qui peuvent être investis génériquement » $(1989: 116)$.

Mais, plus fondamentalement, ce sont surtout les grandes distinctions de la linguistique, et en tout premier lieu la distinction entre synchronie et diachronie, qui permettent d'aborder l'étude d'un genre particulier avec une exigence scientifique satisfaisante, notamment concernant leur évolution (plus bas et infra 5.1.c). On peut citer à cet égard le passage suivant, qui formule selon nous un des savoirs préalables indispensables à toute étude d'un genre particulier :

Dans l'étude des genres, pourtant, on n'est arrivé qu'avec beaucoup de retard à la conclusion que les genres sont à la fois «systématiques" et "historiques" (Glowinski: 1969/74, p. 176). Cela n'a été qu'avec la découverte tardive du structuralisme de l'Europe de l'Est qu'on a su déterminer le genre, par analogie avec les langues historiques, comme un "système en mouvement » (Coseriu : 1958/74, p. 236), c'est-à-dire comme une structure qui est systématique à tout moment de son histoire (l'axe de la synchronie) et qui, par cette même systématicité, est historique (l'axe de la diachronie). Il faut donc analyser les genres soit par leur fonctionnement systématique soit par leur parcours historique. Et c'est le point de vue descriptif qui doit nécessairement précéder le point de vue historique: l'histoire d'un genre compris comme un processus de systématisation permanente ne peut être écrite qu'en se fondant sur de nombreuses coupes transversales faites le long de l'axe diachronique. (Kuon 2015 : 186).

41 En diachronie, plus précisément, la description des genres porte sur les conditions de leur création (par ex. le roman picaresque espagnol), sur leur évolution (le concept de « lignée générique » chez Rastier (2001a : 252)), sur leur co-évolution (Koch 1997) et sur leur éventuelle archaïsation (par ex. le dit médiéval, la criée publique, etc.). Ces objectifs relèvent d'une linguistique historique des genres (Gaberell 2000, Weidenbusch 2006, Hauser 2014), qui appelle le concours nécessaire, mais non suffisant, d'une grammaire de texte historique (Ziegler 2010). Plus précisément, cette tâche linguistique s'applique au phénomène de la variation historique des genres (voir infra 5.4.1). 
42 Mieux, en plus de la distinction entre synchronie et diachronie, la transposition à la problématique des genres de la distinction entre structure et architecture (de la langue ; Coseriu $2001: 239-244^{47}$ ) apparaît non seulement méthodologiquement opératoire, mais théoriquement novatrice, au sens où elle pointe un thème de recherche orphelin en linguistique des genres : celui de la variation générique. En effet,

l'état historique d'un genre ne constitue pas une entité ou structure systématique, mais plutôt une multiplicité ou architecture de systèmes, comparable, là encore, à l'état d'une langue (Coseriu: 1976, p. 27). Ce diasystème peut être structuré du point de vue spatial (diatopique), socioculturel (diastratique) et stylistique (diaphasique). (Kuon 2015 : 187).

43 Ainsi, en plus de la variation diachronique, évoquée plus haut à propos de l'évolution, les genres sont selon Kuon sujets à la variation diatopique (« on pourrait croire que l'étude de genres nationaux est le pendant de la dialectologie en linguistique »), mais aussi à la variation diastratique (roman courtois vs roman picaresque (XVII ${ }^{\mathrm{e}}$ siècle); roman psychologique vs roman d'aventures (XIX ${ }^{\mathrm{e}}$ siècle)). Or cette intuition d'une variation générique n'est pas isolée, bien qu'elle soit d'une grande rareté chez les théoriciens des genres :

J'ai indiqué plus haut que le modèle de la communication dont je me servais ne tenait pas compte du contexte, du lieu et du temps. Or, il existe de nombreux noms de genres qui sont composés à l'aide de déterminants de lieu et de temps. Ainsi des termes comme tragédie élisabéthaine, tragédie classique, roman antique, sonnet baroque, etc. délimitent des traditions dans le temps, c'est-à-dire se réfèrent à des genres historiques au sens le plus fort du terme. (Schaeffer 1989 : 117)

Si Schaeffer, en mentionnant ce qu'il nomme "modulation générique ", évoque essentiellement ici, comme Kuon, des cas de variation interculturelle (comme le chant courtois provençal vs le chant courtois italien, short story irlandaise vs short story américaine, etc.), une telle convergence de vues ne peut qu'inciter à investir plus systématiquement la problématique des genres sous l'angle de la variation en général (infra 4.3 et 4.4) et en particulier sous l'angle de chaque variation « dia- » au sein d'une même culture (infra 5).

\section{Voies de l'étude des genres: conceptions du langage et perspectives empiriques}

L'objet et la méthodologie de la linguistique des genres sont une chose, une autre sont les grandes lignes de partage organisant ce champ de recherches, c'est-à-dire les différentes manières dont les linguistes s'emparent des genres. À ce titre, l'hétérogénéité de ce champ réside dans une multiplicité de points de vue et d'approches $^{48}$ qui ne signifie pas, rappelons-le, « que la théorie des genres n'a pas d'objet, mais que l'objet est toujours relatif à la théorie, qu'il nait de la rencontre des phénomènes et de notre manière de les aborder. » (Schaeffer 1989 : 69).

Pour caractériser l'organisation de ce champ, plusieurs voies peuvent être empruntées, comme en témoignent les synthèses consacrées à la linguistique textuelle (dont Adamzik 2018a, Coseriu 2007, Rastier 2001a). On peut s'en inspirer pour faire ressortir les différentes voies qui sont pratiquées en linguistique des genres, soit deux conceptions du langage opposées auxquelles s'ajoutent deux perspectives distinctes 
selon lesquelles les normes génériques peuvent être considérées et, par conséquent, décrites.

\subsection{Les deux conceptions du langage et la diversité des genres}

La description d'un genre diffère selon qu'elle relève d'une conception logicogrammaticale du langage ou d'une conception rhétorique/herméneutique :

Deux voies s'ouvrent à l'étude des genres [...]. La première étend au texte les voies de l'analyse grammaticale, par des procédures de segmentation, pour constituer une syntagmatique et une théorie des distributions des unités textuelles. [...]. Ces unités sont discrètes et localisables, comme l'atteste, par exemple, le nom de séquence. La conception rhétorique/herméneutique ne nie évidemment pas les régularités, mais ne rapporte pas exclusivement les formes sémantiques à des localisations spatio-temporelles, car ces formes ne sont pas des objets au sens chosiste du terme [...]. Les manifestations des unités textuelles peuvent en effet rester diffuses (isotopies, faisceaux isotopiques) ou rhapsodiques (thèmes) (Rastier 2001a : 236-237).

Cette conception logico-grammaticale sous-tend la plupart des travaux faisant figure de référence en linguistique textuelle de langue française et qui conçoivent le genre à travers le prisme d'unités segmentales de type propositionnel ${ }^{49}$, comme l'illustrent les travaux de Charolles, Combettes ou encore Maingueneau (Portillo 2010, Adam 2010). En particulier, d'une manière assez symptomatique, on peut se souvenir ici que le concept de séquence textuelle (descriptive, narrative, argumentative, explicative, dialogale) développé par Adam (2011: 161-202), un concept parmi les plus influents en linguistique des genres, se revendique de l'article «Text grammar and Text logic » de van Dijk (1973). Plus généralement, cette conception du langage conduit à représenter la diversité des genres comme étant essentiellement organisée par des rapports hiérarchiques, en termes de «classe», de "type» ou de "sous-genre», et plus généralement au moyen de typologies normatives et a priori ${ }^{50}$. Nous y reviendrons (infra 4.5).

Néanmoins, il convient ici d'éviter toute caricature épistémologique et, au sujet précisément de la notion de type, il est important de noter que certaines théories particulières ne se laissent pas exclusivement identifier avec la conception logicogrammaticale. Ainsi alors qu'Adam défend les séquences comme des types d'agencement textuel (opérant exclusivement au niveau transphrasique), il récuse aujourd'hui à bon droit la position extrême, réductionniste, qui consiste à les assimiler à des types de texte (contra Kerbrat/Traverso 2004) :

le concept de types de textes (Texttypen) m'apparait plus comme un obstacle épistémologique que comme un outil heuristique. Les théoriciens et les praticiens qui parlent de types de textes s'inscrivent dans un courant classificatoire qui, à côté de la mise en place des grammaires de textes, a cru pouvoir réduire la diversité des textes à un petit nombre de formes relativement stables. (Adam 2012 : 15)

Cette attitude critique montre la spécificité des propositions récentes d'Adam qui se situent ainsi à la croisée des deux conceptions du langage, comme il le rétablit luimême (Adam 2011: 295-296). Même si sa définition du "genre textuel", qu'Adam oppose au genre discursif (de nature culturelle) ${ }^{51}$, donne quelque peu l'impression de faire retour à un type textuel qui n'en aurait plus le nom.

51 La conception rhétorique/herméneutique, quant à elle, s'illustre tout particulièrement dans les théories du texte qui se donnent pour objet le sens et son interprétation, 
comme la linguistique du sens de Coseriu (2007) ou la sémantique interprétative de Rastier (2001a, 2006), initialement fondée sur le concept d'isotopie (Greimas 1966, Rastier 1987). Ces théories représentent, chacune à sa manière, la seconde sorte de linguistique du texte jadis clairement distinguée par Coseriu (2007 : 7-36). En effet, tout en fondant la légitimité de la grammaire de texte (ou grammaire transphrastique), Coseriu pointait alors les limites de cette dernière pour retenir que la "véritable " linguistique du texte devait être une linguistique du sens, associant une sémiotique textuelle à une herméneutique philologique (Gérard 2019). Sous cet angle sémiotique, au regard duquel l'unité du texte résulte d'un appariement entre plan du contenu et plan de l'expression (Rastier 2006 ; Coseriu 2007 : 64-65), la diversité des genres ne peut que faire l'objet de classifications complexes ou «multi-critères» (ou de "classifications hétérogènes ", selon Petitjean 1989), au nombre desquels il faut compter l'énonciation représentée, le contenu thématique, la formation linéaire du sens textuel (dont les rythmes sémantiques ${ }^{52}$ ) et les fonctions pragmatiques.

De surcroît, héritière de l'ancienne rhétorique (en tant que discipline du trivium) et de la tradition herméneutique ${ }^{53}$, cette conception du langage l'est également de la philologie en ce qu'elle est particulièrement soucieuse de l'historicité des langues et des textes. À ce titre, la tâche du linguiste consiste ici " plutôt à énumérer et à décrire les genres dans leur époque, sans chercher à les subsumer sous des catégories générales » (Rastier 2001a : 238). Rastier (ibid.) et Adam (1999: 94) s'accordent d'ailleurs sur ce point, en revendiquant tous deux une citation de Tomachevski particulièrement emblématique de la conception rhétorique/herméneutique, qui appelle en outre à fonder la description des genres sur une pluralité de critères :

On ne peut jamais établir une classification logique et ferme des genres. Leur distinction est toujours historique, c'est-à-dire justifiée uniquement pour un temps donné ; de plus cette distinction se formule simultanément en plusieurs traits, et les traits d'un genre peuvent être de nature toute différente de la nature de ceux d'un autre genre [...]. Il faut réaliser une approche descriptive dans l'étude des genres et remplacer la classification logique par une classification pragmatique et utilitaire tenant uniquement compte de la distribution du matériel dans les cadres définis. (Tomachevski 1965).

Dans cette optique, la description des genres doit faire de l'historicité de son objet un de ses principes directeurs (supra 2.3). Et, toujours dans cette optique, l'approche variationnelle des genres n'est autre qu'un prolongement naturel de la réflexion historique, qui passe par la reconnaissance du statut culturel des genres (infra 4).

\subsection{Deux perspectives empiriques sur les normes génériques}

À cette divergence de conceptions, située à l'échelle générale du langage, se superpose une différence de perspectives portant sur les normes génériques, qu'on peut traduire par l'opposition entre une perspective idiomatique et une perspective textuelle. En effet, et ce fait aujourd'hui banal constitue d'ailleurs le constat initial des réflexions de Bakhtine (1984 : 265), étant donné que, d'un côté, tout texte (oral ou écrit) se réalise au moyen d'unités puisées dans une langue historique particulière et que, de l'autre, tout texte se présente sous la forme d'une composition linéarisée (traversée par un sens constitué dynamiquement par un acte interprétatif), il est possible soit de concentrer l'étude linguistique sur les rapports d'un genre avec la textualité, soit de la focaliser sur ses rapports avec la langue et ses variétés. Plus précisément, et pour illustration : 
55 1) Perspective textuelle : l'étude des corrélations entre les genres et la textualité présente deux faces distinctes qui correspondent à la dichotomie opérée par Coseriu au sein de la linguistique textuelle (supra 3.1): a) sous l'angle de la grammaire transphrastique, l'influence des genres concerne l'organisation séquentielle des textes (Adam 2011) ou encore les " chaînes de coréférences » (Schnedecker 2014) ; b) sous l'angle de la sémantique des textes, les genres peuvent entre autres conditionner le plan de texte à adopter, la nature rhétorique du titre, la qualité des figures de style, ou encore la thématique de la communication (Adamzik 2004: 126-128), et, au fond, chacune des quatre composantes du sens textuel (Rastier 1989, 2001a).

2) Perspective idiomatique : à la suite de Ferguson (1983), puis surtout de Biber (1988), et d'alternatives à sa méthodologie (Malrieu/Rastier 2001), l'étude des rapports entre les genres et l'usage de la langue s'est développée dans des domaines aussi divers que l'usage du diasystème (Koch/Oesterreicher 2001, Glessgen 2007 : 130, Glessgen/Schøsler 2018), la productivité morphologique (Gérard 2018), l'innovation lexicale et sa diffusion (Vorger 2011, Gérard/Lacoste 2017), le changement linguistique (Koch 2015a et b), ou encore la grammaire (Despierres/Krazem 2012).

Bien entendu, ces deux manières d'envisager les normes génériques ne sont pas exclusives l'une de l'autre : en tant qu'elles rendent compte d'observables distincts, se manifestant dans les textes (oraux et écrits), il s'agit de deux perspectives empiriques qui se complètent en droit et qui peuvent être diversement associées dans la pratique descriptive (comme l'illustre la Figure 2, supra 2.1).

Il est notable à cet égard que Biber/Conrad (2009) figurent parmi les rares chercheurs concevant d'emblée, dans leur modèle, la complémentarité des perspectives idiomatique et textuelle. En soulignant, en effet, que " the same texts can be analyzed from register, genre, and style perspective" (ibid.: 15), ils en résument ainsi les caractéristiques définitoires (que nous commentons en gras, en bas de tableau) :

\begin{tabular}{lll}
\hline $\begin{array}{l}\text { Defining } \\
\text { characteristics }\end{array}$ & Register & Genre \\
\hline Textual focus & $\begin{array}{l}\text { sample of text } \\
\text { exerpt }\end{array}$ & complete texts \\
$\begin{array}{l}\text { Linguistic } \\
\text { characteristics }\end{array}$ & $\begin{array}{l}\text { any lexico- } \\
\text { grammatical } \\
\text { feature }\end{array}$ & $\begin{array}{l}\text { specialized } \\
\text { expressions, } \\
\text { rhetorical } \\
\text { organisation, } \\
\text { formatting }\end{array}$ \\
\hline & Perspective & $\begin{array}{l}\text { Perspective } \\
\text { textuelle }\end{array}$
\end{tabular}

Figure 3 : interprétation de l'opposition entre registre et genre chez Biber/Conrad. 

(textuel) et appliquent par conséquent une conception essentiellement logicogrammaticale, le "registre » et le "genre » correspondent bien aux deux principales perspectives à partir desquelles le linguiste accède aux caractéristiques linguistiques des textes : l'angle du "registre » permet d'observer les phénomènes locaux de type lexico-grammatical et l'angle du « genre » permet de situer l'observation à l'échelle de la structuration globale du texte. Et dans ce second cas, le « genre » de Biber et Conrad renvoie clairement à la notion classique de genre, entendu comme modèle culturel prescrivant, notamment, une certaine typo-disposition du texte, certains motifs rhétoriques, certaines phraséologies, etc. Néanmoins, malgré cette répartition explicite des tâches entre "registre » et "genre ", l'usage de ces deux notions entretient une certaine confusion terminologique ${ }^{54}$, en particulier parce que l'association privilégiée entre certaines caractéristiques lexico-grammaticales et un registre donné (par ex. selon Biber et Conrad, la conversation, le manuel d'école, l'article scientifique, etc. $)^{55}$ relève également, à l'évidence, des conventions culturelles et plus spécifiquement des normes de genre. Il semble par conséquent plus raisonnable de ne conserver ici qu'un seul terme, celui de genre, tout en retenant que sa description linguistique intégrale nécessite l'application d'une double perspective textuelle (globale) et idiomatique (locale).

Ayant délimité l'objet de la linguistique des genres, puis précisé les lignes de partage de son champ d'études, il convient à présent de dépasser le point de vue linguistique stricto sensu, non pour le secondariser, mais pour enrichir sa conception verbale des genres au moyen d'un point de vue ethnologique qui, plus englobant, met clairement en relief les phénomènes de variation générique.

\section{Le statut culturel des genres : un point de vue ethnologique}

\subsection{Les genres au-delà du linguistique : la conception de Günthner/ Knoblauch}

En effet, en elle-même, l'étude linguistique des genres ne permet pas de prendre la pleine mesure de la problématique des genres, car par définition elle réduit à sa dimension verbale un objet d'étude beaucoup plus complexe, comme l'a démontré Thomas Luckmann (e.g. 1992), en sociologie de la connaissance. En particulier, ses travaux se distinguent de ceux de Dell Hymes (Masquelier/Trimaille 2012), généralement considéré par les linguistes comme un des jalons historiques de l'étude des genres :

According to Luckmann (1992), communicative genres fulfil a more crucial function than just representing one possible " component of a speech event " - as Hymes (1974) has claimed. They represent central communicative means in the construction of social reality. [...] Communicative genres fulfil important functions with respect to the coping with, transmission and traditionalization of intersubjective experiences of the life-world. (Günthner/Knoblauch $1995: 5$ ).

Plus précisément, dans ce cadre théorique élargi, Günthner/Knoblauch proposent de distinguer trois niveaux pour analyser les genres communicationnels (« communicative genres ») : 
a) Le niveau de la «structure interne » des genres comprend tous les aspects du langage matérialisés par des signes verbaux (éléments lexicaux, procédés morphosyntaxiques, registres de langue, proverbe, etc.) et non-verbaux (voix, mimique, gestuelle, posture, etc.). Caractériser un genre sous l'angle de la «structure interne » revient donc à appliquer un point de vue sémiotique pour rendre compte $\mathrm{du}$ fait que, lors des échanges communicationnels humains, l'utilisation des signes existants, et également la création de nouveaux signes ou de nouvelles « formes de communication ", passe par une acceptation plus ou moins tacite des conventions (règles ou normes) en vigueur - pour s'y conformer ou pour les contester. Précisément, ce point de vue est celui d'une sémiotique des cultures (Rastier 2001b: 282-284), visant à rendre compte du fait que l'agir humain est en permanence limité par des agencements de signes verbaux et non-verbaux hérités du passé, et pas seulement par des contraintes matérielles et des représentations sociales.

Concernant la seule dimension verbale du langage, il est intéressant de remarquer ici que Günthner et Knoblauch, pour caractériser un genre au niveau de sa "structure interne", non seulement allient la perspective idiomatique à la perspective textuelle (exposées supra 3.2), mais qu'ils allient aussi les aspects responsables de la composition textuelle (plan de texte, séquences, etc.) aux aspects impliqués dans la constitution du sens textuel, comme les figures rhétoriques (tropes) et différentes formes sémantiques (thèmes, motifs, tons, etc.). Sur ce second point, ils conjoignent ainsi en pratique grammaire de texte et linguistique du sens (supra 3.1). Tout ce qu'embrasse ce seul niveau d'analyse suffit déjà pour prendre la mesure du réductionnisme qui touche certaines études en linguistique des genres (et en linguistique tout court). Mais ce n'est pas tout.

b) Le «niveau situationnel » des genres concerne le contexte de l'interaction entre les participants à la communication. Concernant les échanges conversationnels, ce niveau comprend les rituels d'ouverture et la clôture de l'interaction ou les rituels d'accueil et d'adieu, mais aussi les schémas d'organisation séquentielle de la conversation, impliquant par exemple les tours de parole. Sur un autre plan, le statut des participants (orateur/auditeur, maître/élève, directeur/employé, etc.) peut jouer un rôle dans la caractérisation d'un genre, tout comme d'autres circonstances non-linguistiques de la situation (utilisation d'objets particuliers, présence de boissons spécifiques, etc. ${ }^{56}$ ) ou l'occasion sociale de la communication (SaintValentin, réveillon de Noël, départ à la retraite, commémoration nationale, etc.). Plus généralement, l'occasion peut associer une fonction spécifique (communicationnelle, mais aussi symbolique) à un genre donné : chez les Kais de Nouvelle-Guinée, les "contes du temps jadis » sont narrés pendant la saison des semailles, pour favoriser les récoltes ${ }^{57}$.

Pour notre propos, on notera que l'occasion peut entrainer l'inhibition ou l'actualisation de certains traits caractéristiques d'un genre, et donc être la source d'un phénomène de variation pour un genre donné. Les auteurs évoquent ainsi l'exemple du meeting politique aux îles Samoa: "Whereas, in political meetings, lauga [genre samoan] is used in a rather instrumentally oriented mode, in ceremonial events this genre happens to be a prototypical "performance event " with a display of great verbal virtuosity ». (op. cit. : 16).

c) Le niveau de la "structure externe" des genres concerne notamment le milieu (famille, groupe de femme, gang de rue, groupe de loisir, etc.) et la catégorie sociale des acteurs de la communication (homme/femme, enfant/adulte, expert/profane, classe moyenne/classe populaire, etc.). Ainsi l'identité sexuelle est un des traits définitoires des genres communicationnels genrés (ex. dans le Caucase géorgien, le genre du toast, réservé aux hommes, est un moyen de constituer la «masculinité » des membres de la communauté).

On notera, comme ci-dessus pour l'occasion, que la catégorie sociale peut être un facteur de variation pour un genre donné. Par exemple, sur les forums de langue française, la salutation "Salut [la] belle » est exclusivement employée par des femmes s'adressant à des femmes : cette féminisation du genre «conversation à 
bâton rompu » comportant aussi des prescriptions thématiques (grossesse, famille, santé, maison, etc. $\left.{ }^{58}\right)$. De même, les " "Contes de Perrault" sont ainsi, dès le XVIII" siècle, passés dans la rubrique "littérature enfantine" à laquelle ils n'étaient pas du tout destinés». (Adam 2012: 17). Mieux, au sein d'un même milieu culturel, la catégorie sociale peut être caractéristique de genres spécifiques, comme l'illustre au Japon le manga dit shojo, réservé aux jeunes adolescentes, opposé au manga shonen, réservé aux garçons. Enfin, le type de milieu social (et la notion de prestige qui lui est associé) impliqué dans la communication peut lui-même occasionner une variation interculturelle, comme c'est le cas en Chine pour la "thèse de doctorat", relativement à nos conventions occidentales ${ }^{59}$.

Cette conception ethnologique nous rappelle que la problématique des genres, en tant que fait social et plus largement culturel, implique par nature beaucoup plus que ce que son approche linguistique laisse généralement penser. Anti-réductionniste, cette conception montre qu'une pleine approche du genre, considéré en tant qu'objet culturel, nécessite de compléter la caractérisation interne des genres (i.e. décrire les caractéristiques sémiotiques de la "structure interne ») par une caractérisation externe qui décrit dans quelles circonstances situationnelles et sociales il est fait usage de tel ou tel genre. Cependant, la linguistique des genres tend à oublier que leur connaissance empirique appelle, au préalable, des éléments de caractérisation culturelle ${ }^{60}$; l'approche scientifique accuse ainsi un déficit méthodologique, tout comme lorsqu'on utilise un genre sans en avoir caractérisé l'état historique (supra 2.1).

De plus, dans ce cadre anti-universaliste, la mise en évidence de différentes formes de variation générique (intra- et interculturelles) est en soi un motif suffisant pour que la linguistique des genres s'efforce de retrouver la mémoire du programme comparatiste initié par W. von Humboldt (Rastier 2001b: \$53-\$60), si elle prétend gager sa scientificité sur la précision empirique et sur la crédibilité épistémologique. En somme, elle restera insuffisamment " ethnologisée » tant qu'elle continuera de restreindre sa conception des genres à la dimension verbale et tant qu'elle n'aura pas tiré toutes les conséquences du statut culturel des genres.

\subsection{Sémiotique des genres et modèles de la communication}

Relativement à la «structure interne » de Günthner/Knoblauch, il apparait nettement que la problématique des genres relève donc avant tout d'un point de vue sémiotique; problématique dont, par conséquent, la linguistique des genres n'étudie qu'une partie. Ce point de vue sémiotique s'impose à l'évidence lorsqu'il s'agit de décrire les genres dits « de la parole» (Kerbrat/Traverso 2004), impliquant par nature des signes nonverbaux, mais aussi pour traiter pleinement de genres comme l'entretien d'embauche, le journal télévisé, le vaudeville, le stand-up, le slam ou le discours de meeting politique, dont le propre est de se réaliser sous la forme d'une performance polysémiotique (voix, gestuelle, son, image, etc.). Du reste, même les genres imposant une matérialisation uniquement graphique (éditorial, mail professionnel, etc.) n'échappent jamais entièrement à une requalification sémiotique, tout texte pouvant par exemple faire l'objet d'une lecture à voix haute (intime, publique ou sous la forme d'un livre audio).

Pour intégrer cette complexité sémiotique des genres, il faut se doter de modèles de la communication intégrant d'emblée des paramètres anthropologiques (statut de l'interlocuteur, émotionnalité, ancrage actionnel, intensité de la coopération, etc.), comme le modèle de Koch/Oesterreicher (2001; infra 5.1.d) ${ }^{61}$. On peut ainsi entre autres rendre compte, comme le font ces auteurs, de la variabilité occasionnelle des genres (ex. 
la Chanson de Roland chantée par un jongleur la veille d'une bataille ou par un ménestrel pour divertir ${ }^{62}$ ), et surtout disposer d'un cadre conceptuel adapté pour décrire les cas de création de variétés d'un genre lors d'un changement de média (phonique/ graphique), que nous aborderons plus loin (infra 5.4.5).

\subsection{Genres et variation interculturelle}

Mais plus essentiellement encore, il faut réaffirmer que la problématique des genres relève en priorité d'un point de vue ethnologique qui, on le sait, a révélé les limites d'une observation de la langue qui l'isole de sa culture d'appartenance ${ }^{63}$, dénonçant ainsi toute approche logocentrique de faits qui sont intimement liés à des pratiques sociales situées. Ce point de vue nous met par ailleurs en garde contre les dénominations de genre correspondant à une trop grande généralité ("LA conversation», «LE talkshow ", "LE débat", "L'éditorial ", etc.), dans la mesure où les genres sont généralement marqués par des spécificités (linguistiques et non-linguistiques) qui assurent leur fonctionnalité au sein de cultures particulières. Ainsi, culturellement parlant, «le » débat n'existe pas au sens où ce qui est réellement pratiqué ce sont le parliamentary debate (anglais), le débat bouddhiste (tibétain), le débat du second tour (français), etc.

Sous cet angle, le statut culturel des genres se manifeste en premier lieu par le fait que chaque communauté culturelle dispose en propre d'un ensemble de genres ayant été élaborés pour répondre à divers problèmes communicationnels d'ordre émotionnel, rationnel et pratique (Fix 2011). Tout genre a donc nécessairement fait l'objet d'un développement endogène (en relation avec les genres déjà existants, Adamzik 2001) au sein d'une culture particulière, et par suite tout genre peut être dit culturellement spécifique, au sens où il porte la marque d'une culture donnée. Concrètement, et de la manière la plus remarquable, chaque culture possède des genres vernaculaires: le haibun et le nô japonais, le limerick anglais, la chanson zouglou ivoirienne, la bush ballad australienne, le conte tamacahut berbère, le conte dùsávù punu (Gabon), la zarzuela et la tertulia espagnoles, la retahila et la bomba costariciennes, l'Erörterung, le Schwank et le Schlager allemands, la pasquinade à Rome, etc.

À ces genres vernaculaires, qui comptent parmi les plus forts marqueurs de singularisation d'une culture (à l'instar des spécialités culinaires et des particularités vestimentaires), s'ajoutent ceux dont la spécificité se comprend plutôt en termes de variation interculturelle. En effet, de nombreuses études (Fix, Habscheid et Klein 2001; Fix 2011) montrent que des genres, à première vue ordinaires ou standards, sont en réalité marqués par des particularismes locaux (concernant leur rapport à la langue, leur thème/leur composition ou leur fonction communicationnelle/symbolique), comme le $C V$ japonais vs le $C V$ français (vs le Lebenslauf allemand), le testament français vs le testament anglais, le roman picaresque espagnol vs le roman picaresque français (Schaeffer 1989 : 139-140), le sonnet italien vs le sonnet castillan (vs le sonnet anglais), le rap gabonais (Aterianus-Owanga 2012) vs le rap français, le polar scandinave vs le polar nord-américain, l'essay anglais vs le tema italien (vs la dissertation française), le depoimento brésilien vs l'Erlebnisberichte allemand (Castilho Ferreira 2005).

70 Que faire de ces variations? se demandera sans doute le linguiste. Tout d'abord, l'existence même de la variation interculturelle invite la linguistique des genres à la plus grande prudence, contre la généralisation ethnocentrique de ses résultats et, en amont, 
contre les représentations abusivement prototypiques des genres étudiés. En l'occurrence, on devrait une bonne fois pour toutes se défaire de l'image caricaturale « $d u$ sonnet» comme soi-disant forme fixe, comme le démontre Schaeffer (1989: 167-170). Ensuite, en pratique, il est important de noter que ce type de connaissances culturelles a une incidence directe, méthodologique, sur la description linguistique des genres: par exemple, l'analyse lexicale d'un corpus de J-rap appréhendera différemment son objet d'étude selon qu'elle s'est avisée ou non, en amont, des spécificités du rap japonais - qui évite délibérément certains thèmes (problèmes communautaires, discrimination raciale, pauvreté, etc.) et qui est moins agressif et vulgaire que les raps américains et européens.

D'autre part, en aval de la description, les résultats pourront être plus adéquatement interprétés (à l'aune de la culture en jeu), selon le principe que les caractéristiques linguistiques d'un genre ne sont pas déliées de l'environnement culturel (et situationnel) de ce dernier : dans le cas du J-rap, l'absence d'un marquage fort au niveau de la variation diaphasique est, globalement, une manifestation linguistique de la fameuse déférence japonaise. Inversement, c'est-à-dire au lieu de concevoir ces variations comme une connaissance méthodologique préalable, l'analyse linguistique peut en faire un objectif et décrire ainsi les caractéristiques linguistiques d'un genre en contrastant deux cultures, certaines caractéristiques se révélant alors culturellement plus distinctives que d'autres (comme la rubrique « references » du CV anglais). En bref, leçon somme doute banale du comparatisme, inclure la variation interculturelle à la méthodologie de la linguistique des genres a pour effet d'accroître la pertinence de ses descriptions.

\subsection{Genres et variation intraculturelle : notion de variété générique}

L'observation de la variation interculturelle doit être complétée par celle de la variation intraculturelle. De ce point de vue, l'attention portée au statut culturel des genres conduit à examiner la variabilité des genres en s'inspirant du modèle classique de la variation linguistique (diachronie, diatopie, diastratie, diaphasie et diamésie). L'étude des genres adopte ainsi une approche variationniste dont nous exposons ici les motivations, et que nous systématiserons dans la section suivante (infra 5).

\section{A. Variétés génériques}

73 Certes, au sein d'une communauté culturelle, certains genres ne se prêtent pas à l'élaboration de variétés et sont, pour ainsi dire, les seules variétés de leur espèce. En France, il existe ainsi une multitude de genres dont le caractère fortement normalisé (officiel ou institutionnel) neutralise la possibilité de créer des variétés distinctes: le procès-verbal de police, la déclaration d'impôts, l'article du journal officiel (de la République française), l'acte authentique de notaire, la minute (de décision de justice), le commentaire de texte (au baccalauréat), etc. Mais concevoir la problématique des genres sur le modèle de ces genres officiels reviendrait à niveler une réalité culturelle qui ne s'avère ni unifiée ni homogène - à l'image de toute langue.

74 À cette conception simplificatrice, on peut opposer l'observation attentive de la diversité culturelle, en commençant par reconnaître l'inventivité que déploient les membres d'une société pour répondre à d'innombrables problèmes communicationnels - générés par la complexité des rapports sociaux (relatifs à la parenté, aux castes, aux 
statuts, au sexe, etc.), la diversité des représentations (du réel, de l'au-delà, d'autrui, du passé, etc.) et la multitude des pratiques (techniques, spéculatives et spirituelles). Dans ce contexte, la création de nouveaux genres, c'est-à-dire de formes de communication inédites, est une manière de répondre à des problèmes qui se posent soit au niveau global d'une culture soit à l'échelle de groupes sociaux plus spécialisés et plus localisés. La diversification des genres d'une culture se révèle ainsi fortement liée à son développement historique.

Cependant, la problématique des genres ne peut pas être exclusivement conçue en termes de résolution de problèmes, et l'inventivité générique ne se résume pas à la création de genres inédits: si les sociétés créent bien du nouveau pour répondre à l'évolution de leurs besoins, elles diversifient également leurs moyens communicationnels en transformant des formes et des pratiques traditionnelles (rites, motifs visuels, etc.). Il existe ainsi d'innombrables exemples (infra 5 et 6 ) de genres déjà existants qui ont été transposés à un nouvel environnement (géographique, institutionnel, etc.) ou qui ont été adaptés dans un but ludique, utilitaire, etc. Par exemple, l'annonce mortuaire "alsacienne " est une adaptation locale de l'annonce mortuaire standard : publiée dans les quotidiens régionaux du Grand Est, elle témoigne "d'une forte spécificité, d'une véritable "exception culturelle" de l'Alsace-Moselle, reflet de l'histoire de cette région. " (Hamm, dans ce volume) ${ }^{64}$. De même, la fable scolaire n'est pas un nouveau genre, mais une forme transposée de la fable classique qui, en tant que telle, n'est pas fonctionnelle dans un environnement pédagogique.

Ce type d'observation montre qu'un genre est susceptible de varier selon le contexte où il est utilisé et, autrement dit, qu'une culture donnée peut pratiquer un même genre sous des formes diverses. Les membres d'un groupe social ne communiquent donc pas seulement au moyen de genres dont l'usage collectif est relativement uniforme (comme le procès-verbal de police, la minute judiciaire, etc.), mais aussi au moyen de nombreuses variétés génériques (comme la fable scolaire, le manga shojo, l'horoscope de magazine féminin, la comédie de mours antillaise, la plaidoirie de concours, etc.) qui sont chacune l'expression d'un particularisme culturel.

Du point de vue de la théorie variationniste, à l'image des variétés d'une langue, ces variétés génériques se laissent systématiser en ayant recours à de grands axes variationnels (infra 5.1), étant donné qu'un genre ne varie pas seulement au gré de l'histoire d'une culture, mais que ses possibilités de variation concernent également d'autres axes: la portée spatiale, le prestige social, la spécificité situationnelle et l'interaction communicationnelle. En particulier, quel que soit l'axe, tout processus de variation générique s'accompagne d'effets linguistiques (infra 5.4): outre ses particularités non-verbales, toute variété générique se distingue par des particularités touchant l'usage de la langue (par ex. permettre les régionalismes) et la composition du texte (par ex. une taille réduite ou une thématique privilégiée). Enfin, il va de soi que toute variété générique se conforme aux fondamentaux définitionnels de la notion de genre : la conception, la fonction et l'évolution (supra 2.1).

À cet égard, ces cas de variation culturelle doivent être distingués de ce qu'on peut appeler la variation occasionnelle (ou ponctuelle) des genres : on peut penser ici à la pratique individuelle consistant à parodier un genre (différente de la parodie de texte), comme l'« anti-roman" de Charles Sorel, le Berger extravaguant (1627), moquant le roman pastoral, ou le récent La mort par les plantes, Glossaire des plantes toxiques et de leur utilisation (H. Eisendle, 2009), anti-manuel de botanique à l'usage des malfaiteurs. 
Relève également, à notre avis, de la variation occasionnelle la présence récurrente dans les lettres aux proches des poilus d'un lexique des fleurs et d'onomatopées (sifflements de balles, impacts d'obus, etc.) qui proviennent de l'environnement quotidien des combattants (Rézeau 2018: 913-915 et 898-902). Mis en œuvre par la volonté d'un auteur ou conditionné par une situation extra-linguistique particulière, la variation occasionnelle n'atteint jamais le statut traditionnel des variétés génériques qui, elles, sont régulièrement utilisées par les membres d'un groupe social.

\section{B. Variété standard et variété non-standard}

79 À la différence d'une langue, un genre ne comporte pas toujours des variétés, mais quand la variation d'un genre se produit elle s'accompagne généralement, semble-t-il, d'une différenciation axiologique des variétés en présence. Autrement dit, dès lors que l'usage d'un genre n'est plus uniforme parce qu'un groupe social se met à le pratiquer différemment (et régulièrement), en l'adaptant à d'autres besoins et à d'autres circonstances, ce groupe fait émerger une variété qualifiable de non-standard distincte du modèle de départ (ou modèle de référence) - qui peut lui acquérir, pour ainsi dire par rétroaction, le statut de variété standard.

Par exemple, les genres de la presse écrite n'existent pas seulement dans leur variété standard, c'est-à-dire tels qu'ils sont exposés dans les manuels spécialisés et tels qu'ils sont enseignés en école de journalisme. De fait, la rédaction de l'édito des magazines spécialisés (Science \& Vie, Grimper, etc.) est beaucoup moins contraignante que le style formel de l'éditorial des journaux nationaux (Le Monde, La Croix, etc.), qui correspond à la variété standard de ce genre. En tant que variété non-standard, l'édito de magazine d'éditorial possède néanmoins son autonomie et sa fonctionnalité propre, au sein des périodiques illustrés. Dans le domaine littéraire, le manga nekketsu shonen offre un autre exemple de variété standard qui co-existe avec de nombreuses variétés nonstandards. Étiquette commode, « le manga » ne correspond en effet ni à un genre ni à une sorte d'«hyper-genre", mais désigne une myriade de variétés (nekketsu, mecha, pantsu, yaoi/yuri, shojo/shonen, kodomo, josei, ecchi, hentai, etc.) qui ont été élaborées dans des conditions historiques particulières et pour répondre à (ou pour créer) des besoins sociaux particuliers (par ex. relatifs au sexe et à l'âge). Aujourd'hui, parmi ces variétés génériques, en raison du haut prestige et de l'incomparable diffusion de ses œuvresphares (Dragon Ball, One Piece, etc.), le nekketsu shonen peut être considéré comme la variété standard (Ducarme 2018).

81 Ce statut de variété standard doit, selon nous, moins être conçu en termes de prototype abstrait qu'en termes de parangon, soit en tant que variété générique perçue, à un moment donné, comme prééminente par les membres d'une communauté donnée (qu'il s'agisse d'une ethnie, d'une caste, d'une profession, etc.), relativement aux autres variétés (non-standards) que ces mêmes membres reconnaissent. À cet égard, l'approche variationniste ne considérera pas qu'il existe un prototype de "l'horoscope ", mais s'interrogera plutôt, pour une période historique définie, sur l'existence de pratiques standards et non-standards de l'horoscope, en établissant, si elle existe, la variété standard du genre sur la base d'un faisceau de critères concordants (normalisation, diffusion, prestige, etc.). 


\subsection{Sous-genre, variation et modèles de la diversité des genres}

Les genres non-standards sont-ils toutefois assimilables à des « sous-genres" ? Et, plus essentiellement, la notion de sous-genre est-elle compatible avec une approche variationniste des genres ? Il ne semble pas, car il s'agit de deux manières très éloignées de concevoir la diversité des genres.

La notion de sous-genre relève d'une logique de classification (supra 3.1) qui ne se place pas du tout sur le plan des phénomènes concernés par la notion de variation, comme notamment la variation selon l'évolution historique (supra 2.3, infra 5.4.5). En effet, la notion de variation ne sert évidemment pas à concevoir la diversité des genres en appliquant à un domaine de discours (littérature, droit, médecine, etc.) une représentation ordonnée en niveaux (supérieur/inférieur), comme le font par exemple Malrieu et Rastier (2001 : 549) :

Discours

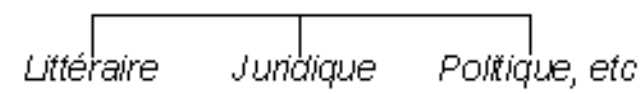

Champs génériques
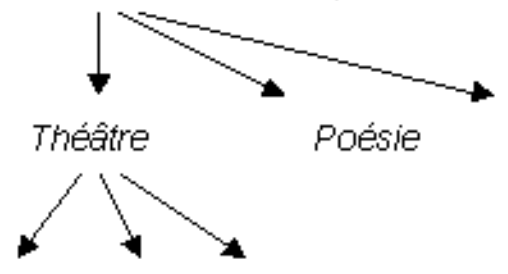

Genres

Comédie Tragédie Drame

Poésie

Récits

\section{Tragede Drame}

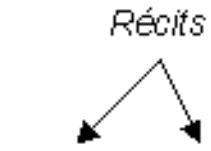

Roman Nowelle

Sous-genres

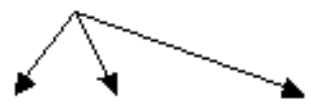

policier de formation par iettres

Figure 4 : une classification des genres (Malrieu/Rastier 2001).

Articulée autour de la notion de sous-genre, cette représentation taxinomique possède une logique propre qui apparait encore plus nettement quand on s'aperçoit qu'il faut compléter ce schéma par un dernier niveau des "sous-sous-genres", de manière à y intégrer toutes les formes bien connues de roman policier: roman d'énigme, roman d'espionnage, polar, thriller, néopolar, polar régional, etc. D'où deux questions : ce processus de différenciation vers le bas de la classification, qui procède par ajout de niveaux, connaît-il une limite rationnelle? Et, surtout, quels critères nous assurent de situer à son juste niveau telle ou telle réalité générique? En quoi, par exemple, n'est-il pas arbitraire de placer «le roman» au niveau des «genres » et le «roman policier» au niveau des « sous-genres" ? Ces questions appellent à l'évidence, de la part de l'étude des genres en général (c'est-à-dire de la part des linguistes, mais pas seulement), une réflexion plus poussée sur l'approche classificatoire de la diversité des genres.

Tout autrement, la notion de variation sert une représentation pour ainsi dire «horizontale ", où la diversité des genres est observée sous l'angle des différentes manières qu'à une culture de percevoir, d'évaluer et de pratiquer des genres, notamment au sein d'un même domaine de discours (journalisme, droit, administration, littérature, etc.). Il ne s'agit donc pas ici d'ordonner des genres au sein d'un espace de représentation abstrait (linnéen), mais d'en décrire l'usage culturel, en 
les observant selon le changement dans le temps, la répartition géographique, la spécificité situationnelle, etc.

L'exemple du roman policier permet d'illustrer cette différence entre une approche variationniste et une approche taxinomique. On sait que «le roman policier» n'est qu'une étiquette commode qui, n'ayant jamais correspondu à un genre historique, recouvre en réalité différentes variétés autonomes qui, génétiquement parlant, ne sont pas également issues d'un même modèle originaire : roman d'énigme, roman d'espionnage, polar, thriller, etc. Chacune de ces variétés correspond à une lignée traditionnelle avec ses œuvres et ses auteurs de référence, mais leur valeur culturelle n'est pas égale. En l'occurrence, avec la diversification historique de ce champ générique, le statut de variété standard s'est vu réassigné : le roman d'énigme (Conan Doyle, Christie, Leroux, Simenon, etc.) a progressivement perdu son statut de standard au profit du polar (Vargas, Minier, etc.) - le polar régional et le néopolar représentant eux, en France, des variétés non-standards de roman policier. À l'opposé, c'est en adoptant un point de vue classificatoire qu'on parle généralement de sous-genres du roman policier (ex. Lits 2002 : §5), sans que soient qualifiées les différences historico-culturelles entre ces sousgenres, au strict niveau de la classification. Du reste, aux yeux des spécialistes, ce qu'on nomme " roman policier » ne renvoie pas à un genre à part entière, c'est-à-dire n'existe pas en tant que genre. À quelle entité d'ordre supérieur se rattachent donc ces sousgenres ? À un contenu prototypique dont les traits résumeraient « le roman policier »? Ou à une simple "étiquette-chapeau » dont le contenu correspond aux différentes variétés historiques? On voit que la notion intuitive de sous-genre soulève une nouvelle fois bien des questions.

Cependant, pour appliquer l'approche variationniste, qui est celle qui s'engage d'emblée et le plus loin dans la reconnaissance du statut culturel des genres, il faut disposer d'un cadre théorique et de concepts opératoires. La section suivante en trace les premiers linéaments en se focalisant sur la variation générique de type intraculturel.

\section{De la variation idiomatique à la variation discursive}

\subsection{Préalables}

Les précédentes réflexions aboutissent à la conception de la variation discursive que nous allons détailler ici, et dont on exposera en conclusion les bénéfices pour la description linguistique des genres, mais aussi pour la constitution ordinaire des corpus (infra 7). Au préalable, toutefois, il convient de formuler quelques remarques au sujet de ce qu'on appelle traditionnellement "variation linguistique ». Ces remarques sont importantes dans la mesure où il s'agit ici de poursuivre le parallèle commencé plus haut entre langue et genre (supra 2.1), en transposant au domaine des traditions discursives des concepts créés pour rendre compte de la variation des langues/ dialectes. Rappelons par conséquent que :

89 (a) La conception des principaux axes diasystématiques (diachronie, diatopie, diastratie, diaphasie) n'est pas exempte de problèmes (e.g. Dufter 2018) et « aujourd'hui il n'existe pas de consensus explicite sur les délimitations précises de ces termes, sur leur interdépendance et sur leur réelle portée théorique » (Glessgen/Kabatel/Völker $2018: 3)$; 
90 (b) Compte tenu de ce constat, notre point de vue théorique, pour penser la variation discursive, est d'adopter les propositions de Glessgen/Schøsler (2018). On admet donc que la structuration des axes diatopique, diastratique et diaphasique prend la forme d'un continuum de type « $\mathrm{X}-\mathrm{Y}$ » (où le $\mathrm{X}$ marque le degré le plus élevé et le $\mathrm{Y}$ le degré le moins élevé) plutôt que celle d'une opposition exclusive de type « $X$ vs $Y$ » (ex. « variété haute» vs "variété basse»). Par suite, l'axe diatopique peut être considéré comme étant structuré par un continuum dont la nature est la portée dans l'espace (fortefaible ${ }^{65}$ ), l'axe diastratique se laisse structurer selon une gradation portant sur le prestige linguistique (élevé-faible) ${ }^{66}$ et l'axe diaphasique se conçoit en termes de degrés de contextualisation, c'est-à-dire de spécificité situationnelle (haute-basse), sur un axe allant du plus dépendant d'une situation de communication particulière (haute spécificité) aux usages les plus routinisés dans une communauté culturelle donnée (spécificité basse).

91 (c) De même, on peut se représenter l'axe diachronique comme un continuum qui, globalement, conduit de l'innovation d'une unité (phonique, lexicale, etc.) à son éventuelle disparition. Plus précisément, on adopte ici la modélisation du changement langagier que Koch présente à propos du lexique (fondée notamment sur coseriu 1978) : «la structure en quelque sorte symétrique des deux versants du changement lexical, logiquement indépendants l'un de l'autre, mais souvent successifs : d'une part le changement innovateur, jalonné par les étapes de l'innovation', de l"adoption' et de la 'diffusion' [Koch 2015a], et d'autre part le changement réducteur - soit la disparition -, jalonné par les étapes de la 'renonciation', du 'refus' et de la 'régression'.» (Koch 2015b : 84). Désignons par évolution historique ce continuum à seuils.

92 (d) Les travaux de Koch/Oesterreicher (2001, à la suite de Söll 1974) ont permis de dépasser les conceptions simplistes de «l'oral » et de «l'écrit ", en modélisant le fait que tout énoncé comporte à la fois un aspect médial (une réalisation phonique ou une réalisation graphique) et un aspect conceptionnel (un «style » d'expression présentant un degré plus ou moins fort d'oralité ou de scripturalité). Ce dernier est conçu comme un continuum communicationnel où se font face un pôle de l'immédiat communicatif et un pôle de la distance communicative. Pour Koch et Oesterreicher, ce continuum sert explicitement à caractériser les genres (i.e. les traditions discursives) du point de vue des paramètres communicationnels qui déterminent la forme que prend l'interaction entre un/des interlocuteur(s) et son/ses/leurs allocutaire(s) :

Le continuum communicationnel [...] se définit, en dernière analyse, par des données anthropologiques qui sont à la base de toute communication humaine. Les recherches pragmatiques, sociolinguistiques et psycholinguistiques nous ont fourni suffisamment de paramètres pour caractériser le comportement communicatif des interlocuteurs par rapport aux déterminants situationnels et contextuels [...] (Koch/ Oesterreicher $2001: 586$, nous soulignons).

Plus précisément, la caractérisation conceptionnelle d'un genre (i.e. la description du « relief conceptionnel » d'un genre, dans les termes de Koch/Oesterreicher), et donc son positionnement sur le continuum en question, repose sur une combinaison des paramètres suivants (pour des exemples, infra 5.4.5) : 


\begin{tabular}{|c|c|}
\hline $\begin{array}{c}\text { Paramètres de l'immédiat } \\
\text { communicationnel }\end{array}$ & $\begin{array}{c}\text { Paramètres de la distance } \\
\text { communicationnelle }\end{array}$ \\
\hline communication privée & communication publique \\
\hline interlocuteur intime & interlocuteur inconnu \\
\hline émotionnalité forte & émotionnalité faible \\
\hline ancrage actionnel et situationnel & détachement actionnel et situationnel \\
\hline ancrage référentiel dans la situation & détachement référentiel de la situation \\
\hline coprésence spatio-temporelle & séparation spatio-temporelle \\
\hline coopération communicative intense & coopération communicative minime \\
\hline dialogue & monologue \\
\hline communication spontanée & communication préparée \\
\hline liberté thématique & fixation thématique \\
\hline$\ldots$ & $\ldots$ \\
\hline
\end{tabular}

Figure 5 : paramètres du continuum conceptionnel (Koch/Oesterreicher 2001 : 586)

genres (supra 4.2), il convient de ne pas restreindre l'aspect médial des productions langagières à la dichotomie réalisation phonique vs réalisation graphique, en la complétant par une dimension polysémiotique (Rastier 2001a: 249) et surtout, en reconsidérant le "graphique » à l'aune des spécificités de l'écrit numérique (Adamzik 2015). Ces aménagements conduisent à parler de médialité sémiotique dont le rôle est central pour la variation interactionnelle (infra 5.4.5).

Or il n'est ici question que de caractériser un genre par des paramètres nonlinguistiques, sans aucune référence à des unités (marqueurs phatiques, interjections, etc.) ou à des constructions (disfluences, inachèvements, etc.) réalisées au moyen d'une langue particulière - comme le font les travaux classiques sur « l'oral » en syntaxe (e.g. Blanche-Benveniste 2010) et, moins classiquement, en sémantique (Missire 2014).

Par conséquent, il conviendrait de concevoir ce niveau de la variation linguistique selon deux axes distincts qui, en fait, correspondent à deux manières différentes d'envisager le même continuum conceptionnel (immédiat-distance). Soit :

i) un axe diamésique (classique, bien que discuté ; e.g. Wüest 2009) qui concerne des unités ou des constructions remarquables et dont le point de vue sur l'oralité/la scripturalité est ainsi en quelque sorte "localiste», mais aussi au sens où sa méthode consiste à se focaliser sur des parties d'énoncés (et non sur la totalité d'un texte);

ii) un axe interactionnel (nommons-le ainsi) de nature englobante (relativement au texte communiqué) qui concerne lui une tradition discursive considérée pour ellemême et dont le point de vue sur l'oralité/la scripturalité ne se situe donc pas à un niveau local, mais au niveau supérieur du comportement communicatif des interlocuteurs.

Convenons que ces deux axes forment les deux voies d'analyse possibles du continuum conceptionnel (de la communication humaine) que, d'une manière cohérente avec les autres continuums de la variation (ci-dessus), nous concevrons à la suite de Koch et Oesterreicher en termes de degré d'immédiat-distance conceptionnel (fort/faible).

Au reste, sur le plan médial proprement dit, pour faire droit au caractère sémiotique des (e) Rappelons enfin que les axes diasystématiques ne sont en aucun cas isolés les uns des autres : ils interagissent de diverses manières (l'idée de "chaîne variationnelle " chez Coseriu (1981), puis Koch/Oesterreicher (1990) ; pour une discussion Glessgen/ Schøsler 2018). Nous exploiterons ce fait plus bas en montrant comment il est possible de comparer différents genres discursifs sur la base des axes de la variation discursive (infra 6). 


\subsection{Les axes de la variation discursive : cadre théorique} diasystématique comme le "contenu» d'une des dimensions fondamentales de la variation linguistique en général - c'est-à-dire abstraction faite de sa relation aux langues/dialectes ou aux traditions discursives, donc à un niveau effectivement plus fondamental. À partir de ces grandes dimensions, il devient possible de concevoir la variation discursive sur le modèle classique de la variation linguistique, en nous représentant la variation discursive comme étant elle aussi constituée d'axes « diatopique », « diastratique ", « diaphasique », « diachronique » et " diamésique » :

\begin{tabular}{|l|l|l|}
\hline $\begin{array}{l}\text { Dimensions } \\
\text { (et continuums) }\end{array}$ & $\begin{array}{l}\text { Axes de la variation } \\
\text { idiomatique } \\
\text { (langues/dialectes) }\end{array}$ & $\begin{array}{l}\text { Axes de la variation } \\
\text { discursive } \\
\text { (traditions discursives) }\end{array}$ \\
\hline $\begin{array}{l}\text { Évolution historique (innovation- } \\
\text { disparition) }\end{array}$ & diachronique & historique \\
\hline Portée spatiale (forte-faible) & diatopique & spatial67 \\
\hline Prestige social (élevé-faible) & diastratique & représentationnel ${ }^{68}$ \\
\hline $\begin{array}{l}\text { Spécificité situationnelle (haute- } \\
\text { basse) }\end{array}$ & diaphasique & situationnel \\
\hline $\begin{array}{l}\text { Immédiat-distance conceptionnel } \\
\text { (fort-faible) }\end{array}$ & diamésique & interactionnel \\
\hline
\end{tabular}

Figure 6 : dimensions, continuums et axes de la variation linguistique.

Ce tableau appelle un certain nombre de commentaires terminologiques. En premier lieu, l'emploi du terme "variation discursive » conduit logiquement à renommer la variation linguistique classique en variation idiomatique, en réinterprétant dans le même temps le terme variation linguistique comme un hyperonyme englobant l'opposition variation idiomatique vs variation discursive. Ces deux créations terminologiques ne sont pas gratuites : dans les faits, d'une part, l'étude de la variation linguistique s'avère généralement restreinte au cas de la variation idiomatique (i.e. concernant exclusivement des faits de langue), d'autre part, la variation discursive (i.e. concernant des genres, notamment) doit être nommée pour mettre en lumière ce thème de recherche orphelin. De surcroît, la pleine observation de la variation linguistique (corrélant ses deux aspects idiomatique et discursif) n'est que rarement mise en œuvre dans les études de cas - alors même qu'elle est un des principaux principes de la diversité culturelle, et par conséquent de l'absence d'uniformité dans les échanges communicationnels humains.

101 En second lieu, concernant la dénomination de ces cinq axes de la variation discursive, étant donné que les phénomènes de la variation idiomatique sont d'une autre nature que ceux de la variation discursive, il convient pour ne pas les confondre de créer une nouvelle terminologie. En effet, une chose est d'observer, par exemple, l'évolution d'un 
fait de langue pour lui-même (innovation phonique, diffusion d'une lexie, etc.), une autre est d'observer l'évolution historique des normes d'un genre donné (ex. les transformations du roman historique). Du reste, choisir de nommer ces axes au moyen de ces qualificatifs (historique, spatial, représentationnel, situationnel et interactionnel) permet d'identifier explicitement un complexe de phénomènes qui passe aujourd'hui largement inaperçu. Nous prenons donc le parti de proposer une terminologie parallèle pour bien distinguer les nouveaux axes désignant la variation discursive des axes traditionnels de la variation idiomatique.

Il va sans dire que ces dénominations sont ici exploratoires et offertes à la critique, et qu'en l'occurrence il est sans doute plus utile d'illustrer, pour terminer cet article, chacun de ces axes par des exemples concrets. Cette illustration permettra spécialement de montrer que ces axes sont non seulement utiles pour décrire la variation générique, mais aussi pour décrire l'identité culturelle de tout genre (infra 5.4 et 6).

\subsection{Utiliser les axes discursifs pour la description des genres}

103 Toutefois, auparavant, il est crucial de comprendre ce principe général de la variation discursive: si chaque genre se laisse caractériser par un certain usage spatial, représentationnel, situationnel, etc. (parce que chaque genre est nécessairement doté d'une identité culturelle, infra 6), tout genre ne fait pas nécessairement l'objet d'un processus de variation. Par exemple, comme nous l'avons évoqué plus haut, certains genres officiels ne se prêtent guère à l'élaboration de variétés (supra 4.4). Donc, en observant une culture donnée à une époque donnée, il faut s'attendre à ce qu'une partie des genres ne soit pas (ou pas encore) concernée par la variation. Autrement dit, et c'est là une particularité essentielle de nos propositions théoriques: chacun des cinq axes permet non seulement de caractériser un genre du point de vue culturel, mais aussi, à la condition que ce même genre ait connu une quelconque variation, de caractériser ses variétés du point de vue linguistique. C'est pourquoi notre illustration des axes adopte, plus bas (infra 5.4), une présentation en deux temps - le premier centré sur l'identité culturelle du genre, le suivant commentant des cas de variation générique du point de vue linguistique.

104 Cette double fonction descriptive des axes discursifs, à la fois linguistique et culturelle, apporte un important bénéfice à la théorie des genres : chacun à sa manière, ces axes permettent d'appliquer une conception relationnelle des genres, qui en effet n'existent pas comme des entités isolées les unes des autres. Ainsi, d'une part, au sein d'un domaine de discours et d'un champ générique, tous les genres se distinguent les uns des autres par des différences d'usage culturel (ci-après et infra 6.2). Par exemple, aux yeux de ses praticiens, la fable-express ${ }^{69}$ possède un prestige moins élevé que la fable classique. D'autre part, concernant la variation discursive proprement dite, la fableexpress (dont la fonction est avant tout ludique) et la fable scolaire (qui remplit des besoins pédagogiques) n'existent que comme des variétés s'opposant à la fable classique - qui conserve le statut de variété standard.

De plus, et c'est là un autre principe général, il ne faut pas s'attendre à ce qu'un genre varie dans sa totalité pour les cinq axes que nous avons définis, mais plutôt à ce que la variation générique n'implique qu'une partie des cinq axes - à l'évidence, par exemple, tous les genres d'une culture ne connaissent pas une variété régionale... On notera aussi que 
les cinq axes de la variation discursive n'ont pas un impact identique. En particulier, la variation historique est beaucoup plus fréquente que la variation spatiale, puisque la plupart des genres voient leurs caractéristiques linguistiques changer, notamment quand ils sont pratiqués pendant une longue période (plus d'un siècle, par exemple). Cette fréquence est une des raisons qui rend plus facile l'illustration de la variation historique des genres que celle de leur variation régionale; et c'est aussi une des raisons qui a conduit les sciences du texte à être essentiellement, voire exclusivement, attentives à la variation historique, au détriment des autres types de variation générique.

\subsection{Application aux genres : usages culturels et variations génériques}

106 1. - Axe historique : d'un point de vue culturel, sur une courte ou sur une longue période, cet axe renvoie à l'évolution historique par laquelle est passé l'usage culturel d'un genre (infra 6), de son innovation ${ }^{70}$ à son éventuelle disparition ${ }^{71}$ en passant par les phases de diffusion et d'éventuelle régression. Parmi d'infinis exemples, mentionnons ceux-ci :

scenegiatta napoletana :

[innovation 1918] - [diffusion 1920-1940] / [régression 1939-1945] ${ }^{72}$ [disparition

1990s]

livre dont vous êtes le héros

[innovation 1960s] - [diffusion 1970-1990] / [régression 1990...]

Du point de vue linguistique, cet axe donne à observer des changements concernant les caractéristiques internes d'un genre - que ces caractéristiques relèvent d'une perspective idiomatique ou d'une perspective textuelle (supra 3.2). Cependant, contrairement aux langues, il faut noter que la variation historique d'un genre peut ne pas avoir lieu, comme le montrent les genres pratiqués pendant une courte période (ex. le roman pastoral, $\mathrm{XVI}^{\mathrm{e}}-\mathrm{XVII}{ }^{\mathrm{e}}$ ). Quand cette variation s'est produite, le genre en question peut devenir l'objet d'une linguistique historique des genres (supra 2.4), comme s'y prêtent les trois exemples suivants : entre son invention en 1949 et aujourd'hui, en France, le journal télévisé (aussi appelé «le $20 \mathrm{~h}$ ») a connu de nombreux changements de forme et de contenu, jusqu'à l'introduction récente de la 4 slow information $»^{73}$; Nicolas Bouvier impulse sa forme moderne au récit de voyage en publiant en 1963 le livre culte L'usage du monde, se distinguant par son style des récits de Chateaubriand et de Montaigne ; enfin, depuis le début du XVIII le genre du sermon a en particulier perdu sa forte codification compositionnelle (Maingueneau 2009).

2. - Axe spatial : d'un point de vue culturel, cet axe permet de caractériser la portée spatiale de n'importe quel genre, et donc de connaître, sous cet aspect, une partie de ce qui définit son usage culturel. Ce fait est loin d'être trivial et l'ethnologie a démontré de nettes corrélations entre usage des genres, espaces communicatifs et moyens linguistiques employés par les locuteurs :

L'étude a porté ici sur une aire culturelle bien spécifique, pour des raisons de cohérence ainsi que de compétence, mais il est probable que beaucoup d'observations faites à propos des genres mandingues sont transposables dans d'autres sociétés. Les types d'organisation spatiale rencontrés ne seront sans doute pas exactement les mêmes, d'autant que les nomenclatures de genres ne coïncident pas nécessairement d'une société à l'autre. Mais le principe qu'il existe, selon les genres ou selon les œuvres du répertoire d'un genre, des régimes spatiaux et que ces 
régimes spatiaux déterminent des enjeux textuels, en termes de fonctions culturelles, restera probablement valable. (Derive 2009 : §61, nous soulignons). alors que l'espace physique associé à l'usage d'un genre peut s'étendre à l'ensemble d'un territoire national, et concerner par conséquent la majorité d'une société ? En effet, historiquement, tout genre initialement très localisé peut voir sa portée spatiale s'étendre en devenant usuel pour une plus grande partie de la population, comme l'opéra bouffe, originaire de Naples, ou les chants du Bwiti, rite initiatique gabonais ${ }^{75}$. Sans trop nous avancer, nous pouvons considérer comme spatialement marqué tout genre dont l'espace physique d'usage s'étend jusqu'à une zone régionale ${ }^{76}:$ au-delà de cette frontière, il paraît problématique de parler de genres spatialement marqués. Quant aux genres non-marqués, ils correspondent selon nous à ceux qui sont pratiqués par la majorité des individus d'une société, comme aujourd'hui, en France, le roman sentimental, le roman de science-fiction, le roman de fantasy, etc. ${ }^{77}$. Mais l'opposition binaire marqué/non-marqué est relativement sommaire et il vaut mieux concevoir l'axe spatial selon un continuum pour rendre compte de tous les cas se situant entre le marquage le plus fort (la pasquinade) et, pour ainsi dire, le degré zéro du marquage (le roman de fantasy). On observe en effet des cas intermédiaires, comme le polar régional de langue française : si ce dernier s'ancre bien dans un territoire précis, en mêlant à son intrigue des éléments d'une identité régionale (toponymes, folklore, usage de régionalismes, etc.), sa pratique est actuellement en vogue dans de nombreuses régions de France, sans qu'il bénéficie de la portée spatiale du polar français contemporain.

point de vue linguistique, pour un genre donné, l'existence d'une variété locale se démontre en décrivant des spécificités (relatives à l'usage de la langue et à la textualité) qui la distingue de la variété standard. Par exemple, la scenegiatta napoletana originale est une variété de mélodrame qui se distingue par des thèmes issus de la culture populaire napolitaine et l'usage du dialecte local (dialogues et parties chantées). De même, le sermon pratiqué à Berne au XVI ${ }^{\mathrm{e}}$ siècle, par sa liberté homilétique, se distinguait de celui pratiqué à Genève par Jean Calvin (Engammare 2016) ${ }^{78}$. Autre exemple, depuis une dizaine d'années, certains dramaturges martiniquais et réunionnais développent un théâtre créole original (Naudillon 2010), dont une comédie de mœeurs antillaise (Naudillon 2009). Cette dernière se laisse en effet concevoir comme 
une variété régionale du répertoire francophone, au sens où "le théâtre des départements d'outre-mer est certainement marqué par le contexte français qui, comme pour toute la littérature écrite, constitue l'arrière-plan incontournable, mais il a su aussi développer une thématique et un ton particuliers. » (Hazaël-Massieux 2000 : 33). De même, suite à l'immigration mandingue en région parisienne (1980-1990), les griots ont dû adapter les genres oraux traditionnels en développant des variétés "parisiennes", marquées par des innovations thématiques et idiomatiques (Derive 2010).

113 Ces deux derniers exemples montrent bien que la réflexion sur la variation spatiale des genres, qui s'inscrit dans les mutations récentes de la sociolinguistique (Auzanneau/ Trimaille 2017: \$22), dépasse la simple localisation physique: elle implique fondamentalement la problématique des identités culturelles (en l'occurrence, pour le théâtre créole, postcoloniales) et, au-delà, l'engagement idéologique de cette approche variationnelle peut consister à "visibiliser les variétés minorisées et les pratiques littératiques non reconnues » (Léglise 2017 : \$16-18) ${ }^{79}$.

114 3. - Axe représentationnel: d'une manière générale, le prestige (élevé ou bas) de n'importe quel objet culturel résulte d'un jugement de valeur qui, en tant que tel, ne peut être que relatif à divers facteurs. Ainsi, le "prestige dont jouit une langue est le "statut fictif", c'est-à-dire sa réputation. Il existe sous la forme de prestige interne, fonctionnant au sein du groupe de locuteurs, et externe, tel qu'il est reconnu par les autres " (Kremnitz: 107). De même, le prestige des genres dépend certes des représentations sociales de son époque (dans l'histoire de la littérature, des «genres mineurs » ont été ultérieurement revalorisés), mais peut au demeurant varier suivant que la réputation d'un genre provient d'un point de vue extérieur à la communauté qui le pratique, ou bien qu'elle se rattache au point de vue des membres de cette même communauté (ce qui nécessite bien entendu, toujours, de définir l'étendue de cette dernière). Ainsi, dans l'actuelle Russie de $\mathrm{V}$. Poutine, le rap conscient (dit aussi rap politique) est tout le contraire d'un genre prestigieux, alors qu'il jouit d'une considération assez forte dans les pays anglophones.

$115 \mathrm{Au}$ sein d'une même culture, un même genre peut également connaître des réputations très différentes selon que le point de vue est porté de l'extérieur ou de l'intérieur : à l'inverse des classes aisées, les classes ouvrières n'auront pas tendance à dévaloriser la chanson paillarde ou le pulp magazine, genres qui constituent une partie de leur fonds culturel. De même, en ce qui concerne les genres de la presse écrite, alors que le fait divers est très apprécié du grand public, aux yeux des journalistes toutefois, sa valeur est inférieure à la chronique judiciaire et il ne détrônera jamais l'incomparable prestige du reportage. Plus précisément, le prestige interne d'un genre, au sein d'un groupe social donné, est toujours relatif aux genres qui lui sont apparentés: dans le milieu académique, la conférence plénière est plus prisée que l'intervention en séminaire de recherche, et l'innovation du cours en ligne (MOOC) n'a pas supplanté le séculaire cours magistral ; dans le milieu du journalisme, le billet ne rivalise pas avec l'éditorial (confié au rédacteur en chef); sur la toile, les internautes estiment moins la conversation par tchat que la discussion sur forum, et raffolent du microbillet (sur Twitter, etc.).

116 Enfin, à l'instar de la variation spatiale, le prestige d'un genre demeure dépendant des aléas de l'histoire : globalement, « la poésie » était plus prestigieuse que « le roman » au $\mathrm{XIX}^{\mathrm{e}}$ siècle (en France), la BD reportage n'a acquis que récemment ses lettres de noblesse, le docudrama historique ne s'impose en France que dans les années 2000 (Garçon 2005), 
etc. Plus généralement, il semble ainsi que la question du prestige dois être pensée en lien avec la fortune historique d'un genre (donc en rapport avec l'axe historique) et qu'elle relève in fine de la réflexion sur la valeur des textes et des œuvres (Rastier 2011, Gérard 2011).

D'un point de vue linguistique, l'axe représentationnel permet de souligner la relation entre une variété « haute » et la/les variété(s) «basse(s) » d'un même genre, en partant de l'hypothèse que la faible réputation d'une variété se manifeste par un certain nombre de particularités linguistiques : un lexique simplifié ou moins valué (argotique, familier, voire vulgaire), une correction syntaxique relative, un plan de texte rudimentaire, une thématique plus prosaïque, etc. On établit ainsi une corrélation entre un aspect de l'identité culturelle d'un genre - son prestige - et sa dimension linguistique. Par exemple, outre sa propension à l'absurde, sa grande liberté thématique et son ouverture à la plaisanterie grivoise, la fable-express se distingue ainsi du modèle de la fable classique (La Fontaine) :

Généralement réduite à un quatrain, cette forme consiste en un bref développement de la trame narrative induite par un calembour appelé «moralité ». De la moralité conventionnelle, cet énoncé ne conserve en fait que des caractéristiques formelles (sa formulation synthétique, sa disposition à la fin de la fable et son isolement typographique). Il procède en général de la déformation d'expressions lexicalisées. [...] Tout se passe comme si la tension classique entre le docere et le placere avait été résolue au seul profit du ridere. (Dupas 2017 : § 8, nous soulignons)

De même, l'opéra buffa se développe au XVIII ${ }^{\mathrm{e}}$ siècle en opposition à l'opéra séria (qui reçoit alors le statut de variété haute) : sans structuration en actes prédéfinie, l'opéra bouffe situe l'intrigue dans la vie quotidienne de milieux populaires où les personnages s'expriment dans un style "bas" (vs sublime) marqué par des traits d'oralité conversationnelle (ex. Orphée aux enfers, Offenbach).

De semblables cas de variation générique, où la variété non-standard «basse » dérive génétiquement d'une variété standard considérée a posteriori comme plus "haute ", sont particulièrement nets, et on pourrait multiplier les analyses de variétés génériques s'autonomisant pour servir une fonction moins prestigieuse que leur modèle générique préexistant; comme le rap parodique, le mockumentaire ou l'éphémère docusoap (Ellis 2010). Comme les autres axes, l'axe représentationnel offre ainsi un poste d'observation possible de la diversification historique des genres d'une culture.

4. - Axe situationnel: l'exemplification culturelle et linguistique de cet axe est particulièrement délicate en raison de la diversité des paramètres qu'il semble possible de convoquer (genre biologique, âge, profession, situation, registre stylistique), dès lors qu'on entend s'inspirer des diverses conceptions de la variation diaphasique (Glessgen/ Schøsler $2018: 25)$. On se bornera à illustrer deux aspects majeurs de cet axe : l'aspect «biologique » et l'aspect correspondant aux pratiques sociales. Ceux-ci résultent de l'application aux genres d'une conception de la diaphasie étendue aux « déterminants biologiques (hommes, femmes, jeunes, enfants) et à des groupes professionnels (Coseriu 1992, 280)» (op. cit. : 21). On élargit toutefois la notion de groupe professionnel à celle de pratique sociale spécialisée (supra 5.1.b), par opposition à une pratique sociale profane. ${ }^{80}$

121 Sous l'angle "biologique » tout d'abord, la caractérisation situationnelle des genres a pour tâche d'identifier les diverses sortes de variétés marquées existant parmi les genres pratiqués au sein d'une culture. Ces variétés marquées, dont le particularisme 
«biologique " peut être corrélé à des particularités linguistiques (voir plus bas), forment en effet un ensemble hétérogène: l'opposition entre manga shojo (pour adolescentes) et manga shonen (pour garçons), l'opposition entre roman d'aventures pour adolescents (marqué) et roman d'aventures "pour adulte» (non marqué), le particularisme féminin du roman sentimental et du chant du gibier gbaya (Roulon-Doko 2013), le particularisme du livre dont vous êtes le héros (pour adolescents) et de la comptine (pour petits enfants). De même, s'il faut distinguer la fable classique (ou de référence) de la fable scolaire (voir plus bas), qui est une variété fonctionnant au sein d'un environnement communicationnel distinct de celui de la fable classique, c'est avant tout parce que la fable scolaire est conçue pour s'adapter aux capacités cognitives de l'enfant, et secondairement pour servir des buts pédagogiques particuliers.

Sous l'angle maintenant de la pratique sociale (spécialisée/profane), le pôle supérieur de l'axe situationnel correspond à des genres marqués par leur "haute spécificité ", c'est-à-dire ceux dont la production et l'interprétation exigent une forte compétence technique, comme l'acte authentique de notaire, l'article de dictionnaire, la chronique judiciaire, etc. La pratique professionnelle de ce dernier genre, en particulier, présuppose en effet, tout à la fois, la maîtrise des techniques journalistiques, la connaissance du droit et du fonctionnement de la justice ainsi qu'un savoir linguistique approfondi ${ }^{81}$ :

le journaliste se doit aussi de relever les mots et les registres de vocabulaire, les façons de s'exprimer de chaque "acteur ": mots du procès (une mère parlant de son fils accusé du meurtre d'une petite fille, qui dit : «cet enfant, je me l'enlève »), les phrases importantes (un avocat général qui lance: "celui-là, il mérite la schlague et le fouet »), les dialogues, les échanges, les réparties et les mimiques qui les accompagnent, les rires, les larmes, les soupirs, les tremblements, ou encore le ton employé par les différents « acteurs » du procès. Certains présidents parlent de façon agressive ou familière aux accusés, d'autres au contraire avec beaucoup de délicatesse. À l'inverse, certains accusés sont desservis par le ton maladroit qu'ils emploient, nerveux, saccadé. (Vernier 2005 : \$31).

À l'inverse, à l'autre bout de l'axe situationnel, les genres marqués par une «spécificité basse ", comme la causerie (conversation quotidienne familière), ne sont ni associés à un domaine de spécialité ni ne requièrent un savoir linguistique techniquement élaboré. Ainsi, une devinette enfantine se caractérise par une spécificité minimale, car les moyens linguistiques en jeu restent ordinaires (français courant, plan question/réponse, thématique ouverte) et parce que cette pratique est entièrement profane. C'est cet angle de la pratique sociale (spécialisée/profane), et non l'angle biologique, que nous appliquerons plus loin dans nos analyses comparées (infra 6.2).

$\mathrm{Du}$ point de vue linguistique à présent, les cas de variation générique peuvent s'observer pour les deux aspects de l'axe situationnel que nous venons d'illustrer, étant entendu qu'il y a variété situationnelle d'un genre quand des particularités linguistiques (lexicales, syntaxiques, thématiques, compositionnelles, etc.) de cette variété ont dû être créées pour adapter un genre de référence (ou genre-source) à un nouvel environnement communicationnel. Ainsi, certains spécialistes de didactique, par exemple, insistent à juste titre pour distinguer la fable scolaire de la fable classique, le conte scolaire du conte populaire traditionnel, le roman d'aventures scolaire du roman d'aventures traditionnel, etc. De fait, seuls ces "genres d'imitation pour apprendre" (Laborde-Milaa $2007: \$ 31)^{82}$, en tant que variétés situationnelles, sont praticables en milieu scolaire et, précisément, ils le sont parce qu'ils aménagent les normes linguistiques des «genres originaux» (ibid.). Cette adaptation du genre à un nouvel 
environnement communicationnel vaut aussi pour la plaidoirie de concours (d'éloquence), variété situationnelle qui donne souvent aux spectateurs une fausse image de la plaidoirie judiciaire (pratiquée par les professionnels et exposée dans les manuels spécialisés). De fait, la plaidoirie de concours se distingue par un temps de parole prédéfini, un sujet et des arguments extra-judiciaires, une liberté diaphasique et, surtout, une théâtralisation où dominent les figures de style mises au service de l'humour.

Tous ces exemples montrent comment la variation situationnelle permet aux cultures d'enrichir l'éventail de leurs modèles communicationnels sans nécessairement passer par la création de nouveaux genres.

5. - Axe interactionnel: nous avons argumenté plus haut pour distinguer cet axe discursif de l'axe diamésique - axes qui selon nous correspondent à deux points de vue complémentaires sur l'immédiat-distance conceptionnel conçu par Koch et Oesterreicher (supra 5.1.d). L'axe interactionnel donne ainsi à observer les genres selon les conditions concrètes de la communication, notamment en ce qui concerne la relation entre un/des interlocuteur(s) et son/ses/leurs allocutaire(s), et en ce qui concerne la relation de tous les participants à leur environnement communicationnel direct. Cet axe reprend exactement la théorisation de Koch/Oesterreicher (2001 : 584-587) qui sert en premier lieu, sur un plan non-linguistique, à rendre compte de l'identité communicationnelle des genres. Par rapport à cette théorisation, l'originalité de notre proposition consiste en une réinterprétation de l'immédiat-distance conceptionnel : nous considérons qu'il s'agit d'un des axes de la caractérisation culturelle des genres, au même titre que les axes historique, spatial, situationnel et représentationnel. En reconnaissant cette fonctionnalité descriptive, nous pensons jeter une nouvelle lumière sur un aspect fondamental de l'immédiat-distance qui, à notre connaissance, reste à l'arrière-plan chez Koch et Oesterreicher.

En pratique, vouloir connaître l'usage culturel d'un genre sous l'angle interactionnel revient à poser les questions suivantes : ce genre implique-t-il que les participants ne se connaissent pas? Qu'ils ressentent une vive émotion dans la situation de communication? Qu'ils soient tenus à distance physiquement (donc aussi symboliquement; ex. le quatrième mur du théâtre) ? Que leurs actes de parole se réfèrent régulièrement à la situation actuelle de communication ? C'est en répondant à ces questions que le linguiste et l'ethnologue, en particulier, peuvent mieux comprendre la dimension interactionnelle des genres qu'ils étudient, complémentairement aux dimensions spatiale, représentationnelle, etc. Par exemple, l'interrogatoire de police est un genre officiel qui se caractérise certes par l'absence d'intimité (entre l'enquêteur et le suspect) et par une thématique relativement fermée (établissement de la vérité au sujet d'une infraction), mais ce genre dialogué se caractérise en même temps par une co-présence spatio-temporelle des participants, une communication relativement spontanée et une forte tension émotionnelle lors des échanges. Autant de particularités qui éloignent l'interrogatoire de police de l'interview radiophonique, de l'entretien semi-dirigé (en sciences humaines et sociales) et, plus nettement encore, de la causerie familière.

D’une manière générale, comme tous les genres historiques présentent un degré d'immédiat-distance conceptionnel, ils prennent nécessairement place le long du continuum proposé par Koch/Oesterreicher (pour une illustration, voir ci-dessous et infra figure 10), quelle que soit la manifestation médiale de ces genres (phonique/ 
graphique-numérique). Sur le plan phonique, par exemple, les dialogues unidirectionnels que sont l'interrogatoire de police (a) et l'entretien semi-dirigé (b) ne présentent pas le même degré d'immédiat conceptionnelle, notamment parce que le second implique une communication plus fortement planifiée (dans son déroulement et jusqu'à la formulation des questions posées), faiblement émotionnelle et prenant la forme d'un dialogue privé. Soit, schématiquement (repères supplémentaires: $\mathrm{c}=$ l'entretien radiophonique; $\mathrm{d}=$ causerie familière ; $\mathrm{e}=$ acte authentique de notaire) :

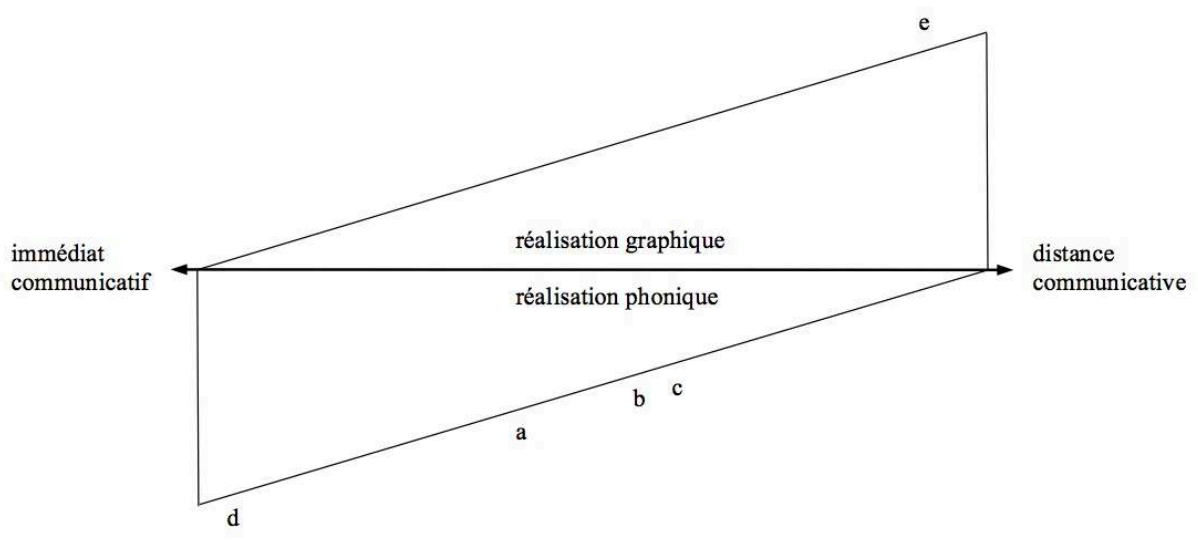

Figure 7 : genres et continuum communicationnel (d'après Koch/Oesterreicher 2001 : 586).

Du point de vue linguistique, la variation interactionnelle des genres présente différents cas de figure, que nous représenterons sur la base du schéma précédent et en reprenant directement les travaux d'Oesterreicher (1993) et de Koch/Oesterreicher (2007). Dans son principe, en effet, la variation interactionnelle peut tout d'abord être "verticale », quand une variété générique ( $a$ et $b$ ) est créée lors de la transposition médiale d'un genre de référence ( $A$ et $B$ ) relevant, au départ, d'un autre type de réalisation médiale; cette transposition s'accompagnant d'un décalage conceptionnel (i.e. par plus d'immédiat ou bien plus de distance; d'où ci-dessous les flèches en biais) :

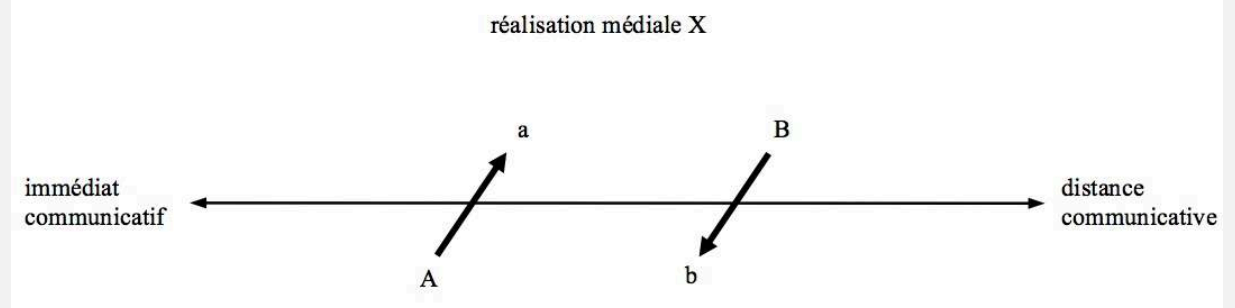

réalisation médiale $\mathrm{Y}$

Figure 8 : les deux cas de variation interactionnelle « verticale ».

Le phénomène bien connu de la scripturalisation des genres oraux (ex. à l'époque médiévale, en Occident, et actuellement en Afrique pour les genres traditionnels), c'està-dire de leur transposition au média graphique, illustre bien cette variation interactionnelle "verticale » et le décalage conceptionnel qui l'accompagne. À notre époque contemporaine, le média numérique se présente à cet égard comme un puissant facteur de variétés génériques. Un exemple récent le montre clairement : vers 2008, la 
nature polysémiotique du réseau social Twitter a conduit à l'invention du twiller (de «twitter » et «thriller ») qui n'est en fait ni un nouveau genre ni un "sous-genre » du roman à suspense, mais bien une variété numérique de celui-ci, dotée de plusieurs spécificités (style fragmentaire, équivoque entre fiction et réalité, interaction avec le lectorat-internaute, usage de photos, etc.) ${ }^{83}$. En dehors du domaine littéraire, on pensera au cours en ligne (MOOC) qui renouvelle le séculaire cours magistral en présentiel. Un autre exemple est celui du journal intime en ligne (Paldacci 2003), variété numérique née de la transposition du journal intime traditionnel (média graphique cursif).

131 La variation interactionnelle peut ensuite être "horizontale ", quand deux variétés d'un même genre (et de même nature médiale) présentent entre elles un écart conceptionnel significatif. Les variétés de l'interview journalistique paraissent offrir un exemple probant, qui illustre en même temps un cas de variation "verticale ». Ainsi, l'interview radiophonique (a), historiquement première ${ }^{84}$, se caractérise par plus d'immédiat conceptionnel que les variétés graphiques correspondantes (nécessitant une réécriture des propos entendus), à savoir l'interview dialoguée sous forme imprimée ( $\mathrm{a}^{\prime}$ : aux yeux des journalistes, la variété standard) et l'interview-récit (a", une variété non-standard). Cependant, l'interview dialoguée conserve une importante part d'oralité, que gomme de son côté l'interview-récit (disparition de l'enchainement des questionsréponses, etc.) :

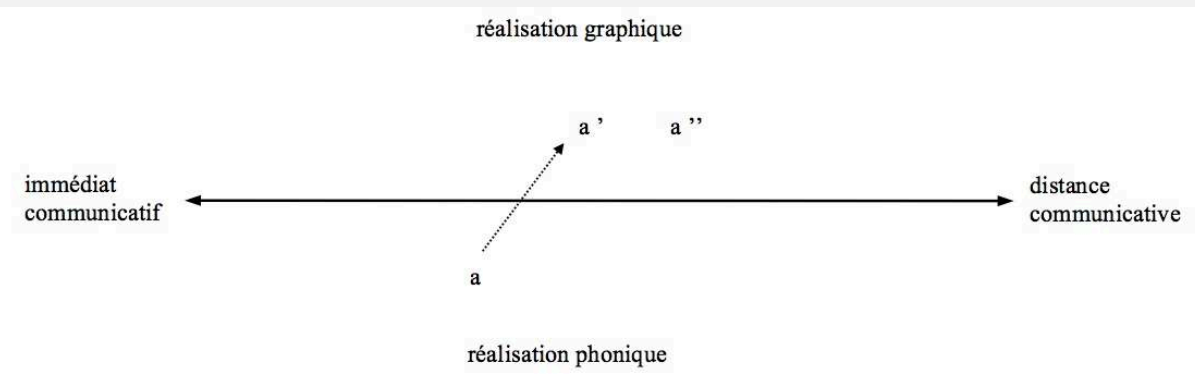

Figure 9 : les deux cas de variation interactionnelle « horizontale».

De même, la chronique judiciaire et la chronique du langage seraient deux variétés de chronique journalistique qui ne s'opposent pas seulement sur la base de leur thématique (judiciaire pour l'un, linguistique pour l'autre), mais qui se distinguent aussi par leur degré d'immédiat-distance, dans la mesure où le ton léger de la chronique du langage appelle un style clairement plus permissif que celui de la chronique judiciaire, qui traite de sujets bien plus graves.

133 Comme pour les autres axes, l'approche variationnelle se signale ici par une conception non-isolationniste des genres qui, en l'occurrence, conduit à décrire l'interview radiophonique dans sa relation à l'interview dialoguée et celle-ci dans sa relation à l'interview-récit. Cette approche, en abordant l'interview journalistique en termes de variété, signale également ce qui relève d'un abus de langage : considérer l'interview radiophonique, l'interview dialoguée et l'interview-récit comme des genres à part entière, trois genres distincts. De fait, reconnaître l'existence de la variation «verticale » permet de faire droit à l'originalité d'une innovation discursive - création d'une variété générique par transposition médiale - en se gardant d'y voir un genre véritablement nouveau. 
Cela étant, pour parler de ces cas de variation interactionnelle, le terme de variété est-il toujours pertinent? En effet, comme la distance communicationnelle entre l'interview dialoguée et l'interview-récit n'est pas fondée sur une différence forte, de nature médiale, on peut se demander si, au lieu de véritables variétés, on n'a pas plutôt affaire à une variation au sens faible, c'est-à-dire à de simples variantes génériques. De même, parmi les formes du portrait journalistique (portrait-CV, portrait-reportage, portrait-enquête, etc.; Mouriquand 2015: \$62-76), laquelle a le statut de variété, laquelle celui de variante? Définir la variante comme une "variété de variété » semble apporter une réponse pertinente et, en même temps, constituer une manière plus fine de penser la variation générique. En l'occurrence, il conviendrait donc de considérer l'interview-récit comme une variante de l'interview dialoguée (en ajoutant, dans la figure 9, une flèche allant de a' vers a") qui, elle, posséderait bien le statut de variété, en raison de sa relation génétique à l'interview radiophonique et de sa différence de nature médiale.

\section{Comparatisme et profil culturel des genres}

\subsection{Le profil culturel des genres : pourquoi ?}

Nous venons de montrer comment, à partir de chaque axe discursif, il est possible de décrire une des facettes de l'usage culturel d'un genre donné, c'est-à-dire à un des aspects définissant la façon dont ce genre est pratiqué par les membres d'une communauté culturelle donnée. En combinant l'ensemble des axes, on peut par conséquent produire une image assez précise de l'usage culturel d'un genre. Selon nous, cette sorte de description, qui consiste à établir le profil culturel d'un genre, devrait être intégrée à la méthodologie du linguiste et de l'ethnologue (infra 7.2) ${ }^{85}$.

D'une part, la caractérisation d'un profil culturel leur permet de s'informer avec une certaine systématicité sur l'objet de sa réflexion: on gagne bien entendu en rigueur méthodologique et en précision interprétative en ayant au préalable compris comment un genre est pratiqué et perçu par ses usagers. D'autre part, au-delà de la caractérisation d'un seul genre, la notion de profil culturel présente l'intérêt fondamental de rompre avec la conception isolationniste des genres, en procurant un outil de comparaison (et donc de compréhension) applicable à des genres apparentés (infra 6.3) ou à des genres appartenant à différentes pratiques sociales (infra 6.2).

\subsection{Caractériser l'usage d'un genre par son profil culturel}

137 À l'instar des axes diasystématiques (supra 5.1, e), les axes discursifs ne fonctionnent pas de manière séparée, mais de manière conjointe, à l'image d'un faisceau. Dans cette optique, l'observation combinée des cinq axes relève de la tâche plus vaste de caractérisation externe d'un genre discursif (supra 4.1, les niveaux "situationnel » et " externe » de Günthner/Knoblauch), qui est complémentaire de leur caractérisation interne, c'est-à-dire linguistique (qui décrit les normes génériques portant sur «la » langue et les composantes de la textualité ; supra 3.2). On se contentera d'illustrer cette caractérisation externe avec certains des genres mentionnés plus haut ${ }^{86}$, en omettant volontairement de préciser leur historicité (supra 2.3 et 5.4.1) et leur nature médiale (phonique/graphique/polysémiotique) : 


\begin{tabular}{|c|c|c|c|c|c|}
\hline 4 & $\begin{array}{c}\text { axe spatial } \\
\text { forte portée spatiale }\end{array}$ & $\begin{array}{c}\text { axe représent. } \\
\text { prestige élevé }\end{array}$ & $\begin{array}{c}\text { axe situationnel } \\
\text { baute spécificité }\end{array}$ & $\begin{array}{l}\text { axe interactionnel } \\
\text { fort immédiat concept. }\end{array}$ & A \\
\hline & $\mathrm{h}$ & $\mathrm{e}$ & c & $\mathrm{h}$ & \\
\hline & \multirow[t]{2}{*}{$\mathrm{c} \mathrm{a} \mathrm{e}$} & & & $\mathrm{g}$ & \\
\hline & & $\mathrm{f}$ & $\mathrm{e}$ & a & \\
\hline & $\mathrm{f}$ & d & $\mathrm{d}$ & $\mathrm{d}$ & \\
\hline & $\mathrm{d}$ & h c & $\mathrm{g}$ & $\mathrm{e}$ & \\
\hline & \multirow[t]{2}{*}{$\mathrm{g}$} & $\mathrm{b}$ & $\mathrm{b}$ & $\mathrm{b}$ & \\
\hline 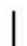 & & a & a & c & \\
\hline & $\mathrm{b}$ & $\mathrm{g}$ & $\mathrm{h}$ & $\mathrm{f}$ & \\
\hline$\downarrow$ & faible portée spatiale & prestige faible & spécificité basse & forte distance concept. & $\downarrow$ \\
\hline
\end{tabular}

Figure 10 : caractérisation comparée de genres (d'après Glessgen/Schøsler 2018 : 33).

Sur l'axe situationnel tout d'abord, l'acte authentique présente le plus haut degré de spécificité, car ce genre est réservé au métier de notaire et présuppose un savoir linguistique techniquement avancé. De ce fait, il s'oppose radicalement à l'annonce mortuaire qui peut être rédigée par tout un chacun et qui possède la simplicité des genres formulaires; ce genre étant lui-même beaucoup plus accessible que la battle rap, dont il faut maîtriser les codes pour oser se mesurer à un adversaire. Sur l'axe représentationnel, la vulgarité et la violence verbale de la battle rap placent ce genre au plus bas, au-dessous de la chanson paillarde qui a cependant moins de prestige que l'annonce mortuaire (ces deux genres s'opposant comme la transgression s'oppose au sacré), cette dernière possédant elle moins de prestige que le slam (plus critique et plus divertissant). Sur l'axe régional, la causerie possède une extension géographique maximale et l'annonce mortuaire alsacienne est la plus localisée, alors que la pratique exclusive du battle rap dans les villes-métropoles en fait un genre beaucoup plus localisé que le slam et que le polar régional ; à la différence de l'acte authentique, de la chanson paillarde et de la conférence scientifique dont la pratique n'est limitée à aucune zone régionale particulière.

Enfin, sur l'axe interactionnel, la causerie présente le plus fort degré d'immédiat conceptionel, car son profil combine la totalité des paramètres de l'immédiat (supra $5.1, \mathrm{~d})$. Au contraire du polar régional dont le degré de distance communicationnelle dépasse celui de l'acte authentique, étant donné que, à la différence du polar, ce genre juridique ${ }^{87}$ est non seulement dépourvu d'émotionnalité, mais aussi que la signature de ce contrat implique la coprésence du notaire et des autres signataires, qui sont des interlocuteurs bien identifiés et non un public d'inconnus ${ }^{88}$. En revanche, l'annonce mortuaire présente une émotionnalité relativement forte qui prend place lors d'une communication publique (parution dans un journal). Quant à la chanson paillarde, ce genre se distingue par un degré amoindri d'immédiat, puisque sa très forte émotionnalité (humour et transgression des interdits) et la nécessaire coprésence spatio-temporelle des interlocuteurs sont atténuées par une thématique fixe (licencieuse) et une communication publique devant des interlocuteurs connus. En revanche, la battle rap possède un ancrage référentiel plus fort, la joute verbale entre ses interlocuteurs prenant de surcroît la forme d'un « dialogue ». 
140 Finalement, chacun de ces genres se laisse ainsi caractériser par un profil qui, à un moment de l'histoire d'une culture, définit sa portée dans l'espace géographique, son prestige social, sa spécificité situationnelle et son degré d'immédiat-distance conceptionnel. L'établissement de ce profil permet de compléter la description de ses particularités linguistiques (caractérisation interne) par la description d'aspects qui nous éclairent sur les conditions précises de l'usage culturel de ce genre (caractérisation externe). Car si, au sein des cultures, les genres sont élaborés pour répondre à des problèmes communicationnels particuliers liés à des situations particulières (Fix 2011), c'est notamment à la caractérisation externe qu'il revient d'en rendre compte.

\subsection{Domaine de discours et comparaison des genres}

141 Au sein d'une culture, l'identité d'un genre dépend de ceux qui l'entourent et c'est précisément ce que montre le tableau précédent, en établissant chaque profil au moyen d'une méthode "différentielle et comparée", à la manière d'une sémiotique des cultures :

Poursuivant un objectif de caractérisation, une sémiotique des cultures se doit donc d'être différentielle et comparée, car une culture ne peut être comprise que d'un point de vue cosmopolitique ou interculturel : pour chacune, c'est l'ensemble des autres cultures contemporaines et passées qui joue le rôle de corpus. (Rastier 2001b: \$59, je souligne).

Par-là, en tant que concept descriptif, le profil culturel offre un accès privilégié à la diversité des genres propre à une culture particulière, tout en fournissant aussi un moyen de comparer des genres appartenant à des cultures différentes.

Toutefois, l'établissement des profils d'un ensemble de genres doit au préalable s'interroger sur la composition d'un tel ensemble. Dans la comparaison précédente (supra 6.2), pour les besoins de notre illustration, nous nous sommes permis de mettre en regard des genres qui ne se côtoient généralement jamais (chanson paillarde et annonce mortuaire, conférence scientifique et battle rap, etc.), parce qu'ils se pratiquent dans des domaines et des circonstances culturellement dissociés. En toute rigueur toutefois, pour être plus adéquate aux multiples divisions et partages sociaux de l'activité communicationnelle, la comparaison devrait être appliquée à des genres fonctionnels au sein d'un même domaine.

144 En guise d'illustration, on peut brièvement montrer comment chaque axe permet de comparer des genres appartenant aux domaines de la presse, de la musique, de la littérature et du spectacle vivant. En premier lieu, sous l'angle de l'axe historique, il est possible d'observer le phénomène de la diversification historique des genres constitutifs d'une même pratique sociale (au sein d'une même aire culturelle) et même le rythme de cette diversification. On note ainsi, dans l'histoire de la presse écrite italienne, des décalages concernant les deux phases d'innovation et de diffusion de ces genres journalistiques (Hrbek 1995, Grosse 2001) :

[innovation] interview (au $20^{\mathrm{e}}$ siècle) - éditorial (dès le 19e siecle) - brève (dès le $17^{\mathrm{e} \text { siècle }}$ )

[diffusion]

145 Ensuite, l'axe spatial peut conduire à constater des différences de portée entre des genres apparentés, comme dans la littérature française contemporaine, entre le roman sentimental, de portée nationale, et le roman régionaliste (dit aussi « de terroir ») (Bois 2014): 
[forte portée spatiale] roman sentimental - roman régionaliste [faible portée spatiale]

élevé]

[prestige faible] chanson paillarde - variété française - chant lyrique [prestige

[prestige faible] intervention - communication - conférence [prestige élevé] ${ }^{89}$

Enfin, l'axe interactionnel donne à observer des différences significatives, en termes d'immédiat-distance conceptionnel, entre des genres appartenant à un même domaine, en l'occurrence la presse écrite et le domaine du spectacle vivant :

[fort immédiat] interview - chronique - éditorial - enquête [forte distance]

[fort immédiat] théâtre de rue - stand-up - match d'improvisation [immédiat affaibli]

Ces différences sont significatives, car elles correspondent à l'emploi, par les locuteurs, de moyens linguistiques distincts. Ainsi, à la grande différence du stand-up, la fort immédiat du théâtre de rue peut se traduire, linguistiquement, par le fait que public apprend le prénom de la personne choisie par les artistes (pour participer au spectacle) et avec qui ces derniers interagissent sur le mode de la conversation quotidienne.

\section{Conclusions}

\subsection{Maniement critique de la notion de genre}

Un des objectifs de cet article a été de réexaminer la complexité de la description linguistique des genres et, conséquemment, de réaffirmer les exigences que devrait remplir l'utilisation méthodique des genres, notamment pour la constitution de corpus. À cet égard, la synthèse de connaissances contenue dans les trois premières sections rappelle que, en linguistique, la pratique de la notion de genre exige une attitude foncièrement critique, en raison des nombreux présupposés conceptuels qui sont nécessairement impliqués dans son maniement.

Cette attitude critique n'a pas seulement pour volonté de contrer la fâcheuse tendance à éluder la définition du genre dans les études y ayant recours : elle vise à expliciter d'autres présupposés qui déterminent tout autant l'orientation et la valeur de la description envisagée. Ainsi, et ce en amont du/des cadre(s) théorique(s) qu'il adopte, le linguiste doit prendre conscience de l'incidence de plusieurs préconceptions :

a) sa conception particulière du genre, relativement à la définition de l'objet idéal de la linguistique des genres (supra 2.1) et à certaines distinctions fondamentales (synchronie/diachronie, structure/architecture, etc.) : l'approche envisagée conçoitelle son objet en termes de genre historique ou de genre théorique/de méthode? Décide-t-elle de se focaliser sur une des trois dimensions du genre (conception, fonction, évolution) ou de privilégier une approche intégrale? Sa reconnaissance du statut culturel des genres inclut-elle la variation discursive, ou demeure-t-elle en deçà ? b) sa conception générale du langage, qui oriente déjà les objectifs de la description et donc la nature des résultats : l'approche envisagée est-elle de type logico-grammatical ou de type rhétorique-herméneutique, ou un alliage des deux?

c) sa perspective empirique sur les normes linguistiques du genre, qui trace une autre ligne de partage en linguistique des genres: l'approche envisagée est-elle 
exclusivement "orientée langue» ou "orientée textualité », ou prévoit-elle une description linguistique intégrale (c'est-à-dire idiomatique et textuelle) des particularités génériques?

d) sa conception de la relation d'appartenance entre le genre et le texte : l'approche envisagée reconnaît-elle les genres inclus, les cas d' "hybridation générique», le rattachement multiple d'un texte à différentes traditions discursives (dont les styles collectifs), etc. ?

Lors de chaque étude particulière, la pratique de la linguistique des genres est ainsi sous-tendue par de nombreux implicites qu'il importe de rendre manifestes pour se garder des approximations méthodologiques. Cette situation devrait plus généralement conduire chaque chercheur à décrire sa propre conception de la linguistique des genres qui, nolens volens, opère nécessairement à l'arrière-plan de sa pratique scientifique individuelle. Nous espérons que les points précédents $(\mathrm{a}, \mathrm{b}, \mathrm{c}, \mathrm{d})$ auront cette utilité.

\subsection{Linguistique, histoire et sociétés}

À cet égard, le point de vue défendu ici est que la linguistique des genres doit viser une ethnologisation maximale de son objet d'étude: identifier puis caractériser un genre particulier, pour l'étudier en lui-même ou pour s'en servir comme d'une donnée en linguistique de corpus, oblige à examiner les conditions de son usage culturel (en commençant par préciser sa culture d'appartenance) pour une période historique définie. Avoir ainsi l'ambition de rester au plus près des réalités historico-culturelles revient par conséquent à privilégier :

a) le genre historique (vs théorique/de méthode) et l'historicité comme principe critique d'identification d'un genre ; un critère crucial qui légitime l'exclusion, hors de la notion de genre, de tout un ensemble de catégories générales (supra 2.3). On privilégie en somme, plus généralement, une conception rhétorique-herméneutique du langage ;

b) une approche sémiotique, reconnaissant la dimension non-verbale des genres (qui les relie à leur situation réelle de production), intégrant d'emblée le rôle de la dimension médiale (supra 5.1 et 5.3.5) et ne dissociant pas les deux perspectives idiomatique et textuelle (à la manière des ethnologues Günthner et Knoblauch, supra 4.1, a) ;

c) une approche variationniste de la diversité des genres (par opposition, en particulier, à une approche taxinomique) qui, contre le préjugé qu'une société fait un usage homogène de ses genres, rend compte du fait que l'usage culturel d'un genre n'est pas nécessairement uniforme, en décrivant les pratiques diversifiées d'un même genre au sein d'une communauté culturelle donnée et pour une période historique donnée ;

d) une approche qui, in fine, fonde l'observation linguistique sur une compréhension culturelle préalable $\mathrm{du} / \mathrm{des}$ genre(s) étudiés, en particulier en caractérisant leur profil culturel au moyen des cinq axes discursifs (supra 5.3 et 6.2) - cette notion de profil permettant de mettre en œuvre, pour la compréhension inter- et intraculturelle des genres, une méthode différentielle et comparée (supra 6.2 et 6.3), partie essentielle de l'héritage de W. von Humboldt.

161 Ce dernier point équivaut à poser en principe que la description linguistique des genres ne peut atteindre sa pleine pertinence qu'en intégrant à sa méthodologie la description de l'usage culturel des genres qu'elle vise à étudier. En effet, alors que l'invention culturelle d'un genre (« oral » ou « écrit ») n'est pas gratuite, mais au contraire toujours 
motivée par des problèmes communicationnels propres à une situation donnée (Fix 2011), l'élaboration d'un genre répond à ces problèmes en fixant des normes qui se composent, notamment, de caractéristiques linguistiques existantes ou créées pour l'occasion (Kabatek 2006 : 287). La linguistique des genres, qui traite précisément de ces caractéristiques-là, ne peut donc les concevoir et les interpréter à leur juste valeur qu'en les reliant systématiquement aux circonstances qui définissent la pratique située de tel ou tel genre - circonstances dont les caractéristiques linguistiques sont génétiquement dépendantes et où elles sont synchroniquement fonctionnelles.

\subsection{Linguistique et variétés génériques}

Pour donner un cadre théorique à l'étude de la variation discursive, dont d'innombrables cas attestent la réalité interculturelle et intraculturelle, nous avons proposé de remonter jusqu'aux dimensions fondamentales qui sont à la base même des différents axes de la variation linguistique classique, pour les appliquer à d'autres objets que les langues. En effet, la grande généralité de ces dimensions autorise leur application aux genres, d'autant plus que ces derniers, comme les langues, sont eux aussi des objets culturels qui, par cette nature même, sont sujets à diverses variations.

Nous avons ainsi proposé de concevoir la variation discursive selon cinq axes (historique, spatial, représentationnel, situationnel et interactionnel), chacun de ces axes s'analysant au moyen d'un continuum spécifique. Il s'ensuit logiquement une requalification terminologique qui se traduit par une extension de sens du terme de variation linguistique, ce terme désignant dès lors deux ordres distincts de phénomènes : la variation idiomatique, portant ordinairement sur les langues, et la variation discursive, portant notamment sur les genres, et plus globalement sur toute grandeur qualifiable de tradition discursive. Globalement, notre visée n'a donc pas été de changer la théorisation classique sur la variation, mais d'en développer la portée au niveau discursif du langage.

C'est aussi pour cette raison que nous avons appliqué la notion de variété, qui en soi n'est pas spécifique aux phénomènes de langue, à la problématique des genres. Par le terme de variété générique nous avons désigné un type d'innovation discursive qui n'équivaut pas à la création d'un nouveau genre (i.e. inconnu du répertoire traditionnel de la culture concernée), mais qui «se borne » à transposer un genre déjà connu à de nouvelles circonstances spatiales, situationnelles, représentationnelles, etc. Dans ce cadre, nous proposons également de parler de variété standard et de variété non-standard pour rendre compte d'une différence de statut qui peut exister entre les variétés d'un même genre.

Pour la linguistique des genres, l'introduction de ces notions nous semble jouer un rôle important, tant sur la manière dont elle conçoit le champ de ses observables que sur le plan du questionnement théorique :

166 a) La notion de variété générique marque en elle-même une rupture avec la conception isolationniste des genres, puisque par nature toute variété est une entité relationnelle. Nos cinq axes discursifs donnent précisément les moyens d'appliquer cette conception relationnelle des genres - qui, loin d'être des entités isolées les unes des autres, sont organisés au sein de champs, de réseaux ou de chaînes (supra 1.1) ;

167 b) La reconnaissance des variétés génériques, en empêchant par exemple de voir de nouveaux genres dans le polar régional, le twiller, la fable-express, etc., prévient le 
linguiste et l'ethnologue contre la tentation de "multiplier les genres ", c'est-à-dire contre l'identification abusive de genres qui n'en sont pas vraiment, au sens strict: certes leur nature est bien historique, mais il s'agit « simplement » de variétés ;

c) L'absence de reconnaissance des variétés d'un même genre peut conduire le linguiste à présenter des résultats en désaccord plus ou moins grand avec les faits, c'est-à-dire avec la diversité culturelle. En effet, sauf dans le cas où la pratique d'un genre est uniforme à un moment donné de l'histoire (ex. l'acte authentique de notaire, le roman pastoral), on ne peut prétendre avoir décrit "le genre X ", c'est-à-dire en avoir fait la description totale, sans avoir pris en compte l'ensemble de ses variétés. Envisage-t-on de décrire le genre de la chronique journalistique? Alors il convient de constituer un corpus comportant des textes de chronique judiciaire, de chronique du langage, etc. De même, on ne peut prétendre décrire "le genre de l'éditorial " à partir d'un corpus principalement composé d'éditos (de magazine), c'est-à-dire à partir d'une seule variété générique. C'est pourquoi, face à la diversité des pratiques génériques, les descriptions partielles, focalisées sur une ou plusieurs variétés historiques d'un même genre, demeurent les moins idéalistes (ou les plus réalistes).

d) La reconnaissance des variétés génériques rend plus précise l'approche discursive de la langue (voir les études mentionnées, supra 3.2.2), en particulier concernant la corrélation bien identifiée (e.g. Koch/Oesterreicher 2001, Glessgen/Schøsler 2018: 32-35) entre genre discursif et variation idiomatique (i.e. diasystème). En effet, l'usage $\mathrm{du}$ diasystème d'une langue varie selon les genres ou, autrement dit, les genres se caractérisent en partie par des permissions et des contraintes concernant l'usage des unités diatopiques, diaphasiques et diastratiques: le sermon contemporain (Metz, Clermont-Ferrand) présenterait une diaphasie modérée et une diastratie plutôt élevée Glessgen/Schøsler : ibid.). Cependant, et sans tenir ici compte du rôle évident de la variation occasionnelle (supra 4.4.1) ${ }^{90}$, deux variétés d'un même genre n'ont pas nécessairement les mêmes implications diasystémiques, ce qui peut se traduire par de fortes divergences. Par exemple, on l'a vu, la différence d'immédiat-distance communicationnel entre l'interview radiophonique et l'interview dialoguée (imprimée) n'implique clairement pas les mêmes permissions et contraintes diasystémiques. De même, le mail amical autorise un degré beaucoup plus faible de diaphasie que le courriel professionnel qui, lui, appelle un lexique plus terminologisé. Et ce phénomène de divergence diasystémique s'observe pour le thriller et le twiller, la chronique judiciaire et la chronique du langage, le conte classique et le conte scolaire, les genres oraux mandingues et les mêmes pratiqués en région parisienne, etc.

$\mathrm{Au}$ fond, alors qu'«ignorer la nature de l'énoncé et les particularités de genre [...] dénature l'historicité d'une étude, [et] affaiblit le lien qui existe entre la langue et la vie (Bakhtine), dénier l'existence des variétés génériques revient à occulter une part essentielle de la linguistique des genres. 


\section{BIBLIOGRAPHIE}

ABLALI, D., BADIR, S., DUCARD, D. (dir.), 2015, En tous genres. Normes, textes, médiations, Louvain-laNeuve, L'Harmattan-Academia. Sciences du langage - Carrefours et points de vue.

ADAM, J.-M., 1999, Linguistique textuelle, Des genres de discours aux textes, Nathan.

ADAM, J.-M., 2005, « Qu'avons-nous fait des espoirs mis dans l'analyse de discours et la théorie du texte ? ", dans C. Jacquet-Pfau, J.-F. Sablayrolles, J. Pruvost, Mais que font les linguistes ? Les sciences du langage, vingt ans après, Paris, L'Harmattan, p. 109-120.

ADAM, J.-M., 2010, « L'émergence de la Linguistique Textuelle en France : entre perspective fonctionnelle de la phrase, grammaires et linguistiques du texte et du discours ", Revista Investigações, 23, p. 39-40.

ADAM, J.-M., 2011, La linguistique textuelle, Paris, A. Colin.

ADAM, J.-M., 2012, « Discursivité, généricité et textualité. Distinguer pour penser la complexité des faits de discours », Recherches, 56, Presses du Septentrion.

ADAM, J.-M., HEIDEMAN, U., 2007, « Six propositions pour l'étude de la généricité », dans R. Baroni, M. Macé (dir.), Le savoir des genres, La Licorne, PUR, p. 21-34.

ADAMZIK, K., 2001, « Die Zukunft der Text(sorten)linguistik. Textsortennetze, Textsortenfelder, Textsorten im Verbund », dans U. Fix, S. Habscheid, J. Klein (dir.), Zur Kulturspezifik von Textsorten, Stauffenburg, Tübingen, p. 15-30.

ADAMZIK, K., 2004, Textlinguistik. Eine Einführung, Tübingen, Niemeyer.

ADAMZIK, K., 2015, « Mediale und konzeptionelle Mündlichkeit/Schriftlichkeit revisited. Geltungsdauer und Gestaltungsaufwand als Operationalisierungskriterien. », dans M. Dalmas et al. (éds), Texte im Spannungsfeld von medialen Spielräumen und Normorientierung, Munich, Iudicium, p. 53-73.

ADAMZIK, K., 2018a, « Textlinguistik », dans F. Liedtke, A. Tuchen (éds), Handbuch Pragmatik, Stuttgart, J. B. Metzler, p. 53-64.

ADAMZIK, K., 2018b, « Derselbe Text, aber anders. Was können Variations- und Textlinguistik von- und miteinander lernen? », dans K. Adamzik, M. Maselko (éds),VARIATIONslinguistik trifft TEXTlinguistik, Tübingen, Narr, p. 19-60.

ADAMZIK, K., 2018c, « Was ist ein Text? », dans K. Birkner, N. Janich (éds), Text und Gespräch, Berlin/Boston, de Gruyter, p. 26-51.

ATERIANUS-OWANGA, A., 2012, « Des diables, des prophètes et des banzis sur les scènes de Libreville : les réemplois religieux des rappeurs du Gabon ", Autrepart, vol. 60, n 1, p. 39-53.

AUZANNEAU, M., TRIMAILLE, C., 2017, « L'odyssée de l'espace en sociolinguistique », Langage et société, vol. 160-161, $\mathrm{n}^{\circ}$ 2, p. 349-367.

BAKHTINE, M., 1984 [1952-1953]), « Les genres du discours », Esthétique de la création verbale, Paris, Gallimard.

BALLABRIGA, M, MPONDO-DICKA, P. (dir.), 2007, Rythme, sens et textualité, Toulouse, Éditions Universitaires du Sud.

BARONI, R., MACÉ, M., 2007, « Avant-propos », Le savoir des genres, La Licorne, PUR, p. 7-20. 
BAUMAN, R., 1999, « Genre », Journal of Linguistic Anthropology, 9, p. 84-87.

BAUMAN R., 2002, « The ethnography of genre in a Mexican market: Form, function, variation », dans P. Eckert, J. Rickford (dir.), Style and Sociolinguistic Variation, Cambridge, Cambridge University Press, p. 57-77.

BEACCO, J.-C., 2004, « Trois perspectives linguistiques sur la notion de genre discursif », Langages, $\mathrm{n}^{\circ} 153$, p. 109-119.

BEAUGRANDE DE, R., DRESSLER, W., 1981, Introduction to Text Linguistics, London, Longman. BIBER, D., 1988, Variation across Speech and Writing, Cambridge, Cambridge University Press. BIBER, D., CONRAD, S., 2009, Register, genre, and style, Cambridge, Cambridge University Press.

BLANCHE-BENVENISTE, C., 2005, « L'étude de la morphologie et de la syntaxe de la langue », dans C. Jacquet-Pfau, J.-F. Sablayrolles, J. Pruvost (éds), Mais que font les linguistes ? Les sciences du langage, vingt ans après, Paris, L'Harmattan, p. 73-92.

BLANCHE-BENVENISTE, C., 2010, Le français, usages de langue parlée, Leuven, Peeters.

BONHOMME, J., 2018, « Incident autour d'un poteau. Rituel, script et performance dans le Bwiti du Gabon », L'Homme, vol. 227-228, n 3, p. 153-178.

BOIS, G., 2014, « Ancrage local et visibilité littéraire. Le cas des écrivains peu reconnus de la région Rhône-Alpes ", Ethnologie française, vol. 44, n 4, p. 621-629.

BOUILLON, C., 2007, « Genre prescrit, genre construit : le conte et ses variations », Le français aujourd'hui, vol. 159 , no. 4, p. 65-74.

BOUQUET, S., 2004, « Linguistique générale et linguistique des genres, introduction au numéro) », Langages, 153, p. 3-14.

BOUQUET, S., GRILLO, S., 2007, « Introduction », Linx [En ligne], 56, mis en ligne le 18 février 2011, consulté le 15 novembre 2018. URL : http://journals.openedition.org/linx/352

BRANCA-ROSOFF, S., 2007, « Genres et activité langagière : l'exemple des tchats », Linx, 56, p. 127-141.

BRINKER, K., 2001, Linguistische Textanalyse. Eine Einführung in Grundbefriffe und Methoden, Berlin, Schmidt.

CALAME-GRIAULE, G., 1965, Ethnologie et langage : la parole chez les Dogon, Paris, Gallimard.

CARTIER, E., 2019, « Neoveille, plateforme de repérage et de suivi des néologismes en corpus dynamique », Neologica, 13, Paris, Classiques Garnier.

CASTILHO FERREIRA DA COSTA, A., 2005, Sozial- und kulturspezifischer Textsortengebrauch. Ein Vergleich brasilianischer und deutscher Schulaufsätze, thèse de doctorat, Université Martin Luther, Halle-Wittenberg.

CIAPUSCIO GUIOMAR, E., 2007, « Genres et familles de genres : apports pour l'acquisition de la compétence générique dans le domaine académique », Ela. Études de linguistique appliquée, $\mathrm{n}^{\circ} 148$, p. 405-416.

COSERIU, E., 1971, « Thesen zum Thema « Sprache und Dichtung » ", dans W.-D. Stempel, (éd), Beiträge zur Textlinguistik, Munich, p. 183-188.

COSERIU, E., 1978, Sincronía, diacronía e historia. El problema del cambio lingüístico, Madrid Gredos, Biblioteca románica hispánica, II, 193). 
COSERIU, E., 1981, « Los conceptos de 'dialecto', ‘nivel' y 'estilo de lengua' y el sentido propio de la dialectología », Lingüística española actual 3, p. 1-32.

COSERIU, E., 1988, « Die Ebene des sprachlichen Wissens. Der Ort des « Korrekten » in der Bewertungsskala des Gesprochenen », dans J. Albrecht (éd.), Energeia und Ergon, Schriften von Eugenio Coseriu, p. 327-364.

COSERIU, E., 2004, « Mon Saussure », dans R. van Deyck, R. Sornicola, J. Kabatek (éds), La variabilité en langue. Langue parlée et langue écrite dans le présent et dans le passé, Communication \& Cognition, Gent, P. 17-24.

COSERIU, E., 2007 [1980], Textlinguistik. Eine Einführung, Tübingen, Narr.

CUSSET, C., 2016, « L'epyllion hellénistique : une forme poétique en quête d'elle-même.

Recherches sur les données métapoétiques de l'epyllion », Aitia [En ligne], 6, mis en ligne le 16 juin 2016, consulté le 28 janvier 2019. URL : http://journals.openedition.org/aitia/1376

DAL, G., 2003, «Productivité morphologique : définitions et notions connexes », Langue française, $\mathrm{n}^{\circ} 140$, p. 3-23.

DE GEEST, D., 2006, « Lectures, post-)modernes de la littérature régionale en Flandre », Études Germaniques, vol. 244, n 4, p. 523-539.

DENNES, M., 1997, « Bakhtine, philosophe ? », dans C. Depretto (dir.), L'héritage de Bakhtine, Paris, PUB.

DESPIERRES, C., KRAZEM, M., 2012, Quand les genres de discours provoquent la grammaire... et réciproquement, Limoges, Lambert-Lucas.

DERIVE, J., 2009, « Typologie et fonctions de quelques genres oraux du Manding à l'aune du critère de la spatialité », Journal des africanistes, 79-2, p. 201-222.

DERIVE, J., 2010, « Diaspora mandingue en région parisienne et identité culturelle. Productions de littérature orale en situation d'immigration », Journal des africanistes, 80-1/2, p. 237-250.

DIJK, T. A. van, 1973, « Text grammar and Text logic », dans J. S. Petöfi, H. Reiser (éds), Studies in Text Grammar, Dordrecht, Reidel, p. 17-78.

DUCARME, F., 2018, « Are nekketsu shōnen manga sports manga? », Comicalités [En ligne], Bande dessinée et sport : stylisation, émancipation, contestation d'un univers normé ?, mis en ligne le 17 novembre 2018, consulté le 02 avril 2019.

DUFTER, A., 2018, « Repenser la "spatialisation" de la linguistique variationnelle », dans Glessgen M., J. Kabatek, H. Völker (éds), Repenser la variation linguistique - repenser la linguistique variationnelle, Actes du colloque DIA IV, 12-14 septembre 2016, Zurich), Société de Linguistique Romane/Éditions de linguistique et de philologie, Strasbourg, p. 63-73.

DUPAS, S., 2017, «Le “pouvoir des fables" à l'épreuve de la modernité : l'exemplarité littéraire en question à la fin du XIXe siècle », Littérature et exemplarité [en ligne], Rennes, Presses universitaires de Rennes, consulté le 07 mai 2019).

EBELING, G., 1959, « Hermeneutik », dans K. Galling (éd), Die Religion in Geschichte und Gegenwart, Tübingen.

ELLIS, J., 2010, «Quand le documentaire rencontre la fiction : la crise du factuel à la télévision britannique en 1999 », Le Temps des médias, vol. 14, n 1, p. 109-123.

ENGAMMARE, M., 2016, « La forme de la prédication à Berne au xvi ${ }^{e}$ siècle », Revue historique, vol. 678 , no. 2 , p. 33-58. 
FERGUSON, C. 1983, « Sports announcer talk: syntactic aspects of register variation », Language in Society, 12, p. 153-172.

FERRY, M.-P., 1970, « Sapir et l'ethnolinguistique », Langages, 18, p. 12-21.

FIX, U., 1997, « Kanon und Auflösung des Kanons. Typologische Intertextualität - ein “postmodernes“ Stilmittel », dans G. Anto, H. Tietz (dir.), Die Zukunft der Textlinguistik. Traditionen, Transformationen, Trends, Tübingen, Max Niemeyer Verlag, p. 96-108.

FIX, U., 2011, « Was ist kulturspezifisch an Texten? Argumente für eine kulturwissenschaftlich orientierte Textsortenforschung », Russische Germanistik. Veröffentlichungen des Russischen Germanistenverbandes, Band VIII, Moscou, p. 172-183.

FIX, U., HABSCHEID, S., KLEIN, J. (éds), 2001, Zur Kulturspezifik von Textsorten, Stauffenburg, Tübingen.

FLYDAL, L., 1951, « Remarques sur certains rapports entre le style et l'état de langue », Norsk Tidsskrift for Sprogvidenskap, 16, p. 241-258.

FREYERMUTH, S., 2011, « Un genre peut en cacher un autre : une histoire de détournement », Linx, 64-65, p. 173-187.

GABERELL, R., 2000, « Probleme einer deutschen Textsortengeschichte », dans Kirsten Adamzik, éd.), Textsorten. Reflexionen und analysen, Tübingen, Stauffenburg, p. 155-174.

GARÇON, F., 2005, « Le documentaire historique au péril du « docufiction » », Vingtième Siècle. Revue d'histoire, vol. 88, $\mathrm{n}^{\circ}$ 4, p. 95-108.

GÉRARD, C., 2008, " Genre et variations stylistiques en sémantique textuelle », dans J. Durand, B. Habert, B. Laks (éds), Congrès Mondial de Linguistique Française, 1er Congrès mondial de linguistique française, CMLF-08), Paris.

GÉRARD, C., 2010, « L'individu et son langage : idiolecte, idiosémie, style », PhiN, Philologie im Netz, 51, http://web.fu-berlin.de/phin/.

GÉRARD, C., 2011, « Herméneutique de la valeur, I). Distinctions élémentaires pour l'étude axiologique des textes. », dans C. Chollier (dir.), Qu'est-ce qui fait la valeur des textes ?, Reims, Éditions et Presses Universitaires de Reims, p. 119-180.

GÉRARD, C., 2016, « Comment juge-t-on les innovations linguistiques ? Typologie intégrale du jugement lexicale », dans C. Jacquet-Pfau et J.-F. Sabayrolles, La Fabrique des mots français, Paris, Lambert-Lucas, p. 363-380.

GÉRARD, C., 2018, «Variabilité du langage et productivité lexicale : problèmes et propositions méthodologiques », Neologica, 12, Paris, Classiques Garnier, p. 23-45.

GÉRARD, C., 2018, «Variabilité du langage et productivité lexicale : problèmes et propositions méthodologiques », Neologica, 12, Paris, Classiques Garnier, p. 23-45.

GÉRARD, C., 2019, « Art du langage et linguistique du sens. Traduction commentée des “Thèses sur le thème 'Langage et poésie”", Eugenio Coseriu) », Pratiques [En ligne], 179-180, mis en ligne le 31/12 2018, consulté le 19 mars 2019. URL : http://journals.openedition.org/pratiques/4918

GÉRARD, C., LACOSTE, C., 2017, « La création lexicale dans les écrits de combattants de la Grande Guerre. L'approche dictionnairique de la néologie à l'épreuve des textes », dans G. Siouffi (dir.), La langue sous le feu, Rennes, Presses universitaires de Rennes, p. 175-192.

GÉRARD, C., BRUNEAU, L., FALK, I., BERNHARD, D., ROSIO, A.-L., 2017, « Le Logoscope : Observatoire des innovations lexicales en français contemporain », dans J. García Palacios et al. 
(éds), La neología en las lenguas románicas : Recursos, estrategias y nuevas orientaciones, Frankfurt am Main, Lang, p. 339-356.

GLESSGEN, M., 2012, Linguistique romane. Domaine et méthodes en linguistique française et romane, Paris, Armand Colin.

GLESSGEN, M., SCHØSLER, L., 2018, « Repenser les axes diasystématiques : nature et statut ontologique », dans M. Glessgen, J. Kabatek, H. Völker (éds), Repenser la variation linguistique repenser la linguistique variationnelle, Actes du colloque DIA IV, 12-14 septembre 2016, Zurich), Société de Linguistique Romane/Éditions de linguistique et de philologie, Strasbourg, p. 11-52. GLESSGEN, M., KABATEK, J., VÖLKER, H. (éds), 2018, Repenser la variation linguistique - repenser la linguistique variationnelle, Actes du colloque DIA IV, 12-14 septembre 2016, Zurich), Société de Linguistique Romane/Éditions de linguistique et de philologie, Strasbourg.

GREIMAS, A.-J., 1966, Sémantique structurale : recherche de méthode, Paris, Larousse.

GRONDIN, J., 2005, « Hermeneutics », dans M. C. Horowitz (dir.), New Dictionnary of the History of Ideas, vol. 3, Thomson Gale, New York/New Haven.

GROSSE, E.-U., 2001, «Évolution et typologie des genres journalistiques », Semen [En ligne], 13, consulté le 17 octobre 2017. URL : http://semen.revues.org/2615

GUILLOT-BARBANCE, C., PINCEMIN, B., LAVRENTIEV, A., 2017, « Représentation de l'oral en français médiéval et genres textuels », Langages, 208, p. 53-68.

GÜLICH, E., 1986, « Textsorten in der Kommunikationpraxis », dans W. Kallmeyer, Kommunikationstypologie, Schwann.

GÜNTHNER, S., KNOBLAUCH, H., 1995, « Culturally Patterned Speaking Practices - the Analysis of Communicative Genres », Pragmatics, Benjamins, p. 1-32.

HANKS, W. F., 1987, « Discourse genres in a theory of practice », American Ethnologist, 4, p. 668696.

HAUSER, S., 2014, « Netze im Wandel - Wandel in Netzen. Diachrone Perspektiven auf die Vernetztheit von Textsorten ", dans S. Hauser et al. (éds), Musterwandel - Sortenwandel. Aktuelle Tendenzen der diachronen Text(sorten)linguistik, Bern, Peter Lang, p. 269-306.

HAUSER, S., KLEINBERGER, G., FIX, U., SVEN ROTH, K., 2014, Textmuster und Textsorten im Wandel Einführende Anmerkungen zu aktuellen Fragen der diachronen Textlinguistik, dans S. Hauser et al. (éds), Musterwandel - Sortenwandel. Aktuelle Tendenzen der diachronen Text(sorten)linguistik, Bern, Peter Lang, p. 7-14.

HAZAËL-MASSIEUX, M., 2000, «Le théâtre créolophone dans les départements d'outre-mer. Traduction, adaptation, contacts de langues ", L’Annuaire théâtral, 28, p. 21-34.

HEIDMANN, U., ADAM, J.-M., 2010, Textualité et intertextualité des contes, Paris, Classiques Garnier.

HRBEK A., 1995, Vier Jahrhunderte Zeitungsgeschichte in Oberitalien. Text-, sprach- und allgemeingeschichtliche Entwicklungen in der « Gazzetta di Mantova » und vergleichbaren Zeitungen, Tübingen, Niemeyer.

HYMES D., 1974, « Ways of speaking », dans R. Bauman \& J. Sherzer (éds), Explorations in the Ethnography of Speaking, Cambridge, Cambridge University Prcss, p. 433-451.

IRVINE J. T., 2001, «"Style" as distinctiveness: the culture and ideology of linguistic differentiation », dans J. R. Rickford, P. Eckert (éds), Style and Sociolinguistic Variation, Cambridge University Press. 
KABATEK, J., 2006, « 'Bedeutungsausbau' und Corpora », dans D. Wolf et al. (éds), Lexikalische Semantik und Korpuslinguistik. Gedenkschrift für Horst Geckeler, Tübingen, Narr, p. 281-300.

KABATEK, J., 2015a, « Genre textuel et traditions discursives », dans C. Gérard, R. Missire (dir.), E. Coseriu : réceptions contemporaines, Éditions Lambert-Lucas, Limoges, p. 195-206.

KABATEK, J., 2015b, « Wie kann man Diskurstraditionen kategorisieren ? », dans A. López Serena, A. Octavio de Toledo, E. Winter-Froemel (éds), Diskurstraditionelles und Einzelsprachliches im Sprachwandel, Tradicionalidad discursiva e idiomaticidad en los procesos de cambio lingüístico, Tübingen, Narr, p. 51-65.

KERBRAT, C., TRAVERSO, V., 2004, « Types d'interactions et genres de l'oral », Langages, 153, p. 41-51.

KLOSS, H., 1978 [1952], Die Entwicklung neuer germanischer Kultursprachen seit 1800, Pädagogischer Verlag Schwann, Düsseldorf.

KOCH, P., 1997, « Diskurstraditionen : zu ihrem sprachtheoretischen Status und ihrer Dynamik », dans B. Frank, T. Haye, D. Tophinke (éds), Gattungen mittelalterlicher Schriftlichkeit, Tübingen, Narr, p. 43-79.

KOCH, P., 2015a, « La structure générale du langage et le changement langagier », dans C. Gérard, R. Missire (dir.), E. Coseriu : réceptions contemporaines, Limoges, Éditions Lambert-Lucas, p. 95-128.

KOCH, P., 2015b, « Disparition lexicale, variétés linguistiques et traditions discursives », dans C. Badiou-Monferran, T. Verjans (éds), Disparitions. Contributions à l'étude du changement linguistique, H. Champion, coll. « Linguistique historique », p. 68-88.

KOCH, P., OESTERREICHER, W., 1990, Gesprochene Sprache in der Romania. Französisch, Italienisch, Spanisch, Tübingen, Niemeyer, $2^{\mathrm{e}}$ éd., revue et augmentée, 2011.

KOCH, P., OESTERREICHER, W., 2001, « Langage parlé et langage écrit », dans G. Holtus, M. Metzeltin, C. Schmitt (éds), Lexikon der romanistischen Linguistik, Tübingen, Niemeyer Verlag, p. 601-604.

KOCH, P., OESTERREICHER, W., 2007, « Schriftlichkeit und kommunikative Distanz », Zeitschrift für germanistische Linguistik, 35(3), p. 346-375.

KRAZEM, M., 2011a, « Présentation : du genre vers la grammaire », Linx, 64-65, p. 7-14.

KRAZEM, M., 2011b, « Représenter les relations entre grammaire et genres de discours : l'exemple des commentaires sportifs », Linx [En ligne], 64-65, p. 45-68.

KRAZEM, M., 2015, « Les genres de discours créent-ils une grammaire exceptionnelle?», Pratiques [En ligne], p. 167-168, mis en ligne le 01 avril 2016, consulté le 19 mai 2017. URL : http:// pratiques.revues.org/2744

KREMNITZ, G., 2013, « Questions de terminologie et de concepts », dans G. Kremnitz, dir.), Histoire sociale des langues de France, Presses universitaires de Rennes, p. 103-112.

KUON, P., 2015, « Les possibilités et les limites de l'étude structurale des genres », dans C. Gérard, R. Missire (dir.), E. Coseriu : réceptions contemporaines, Limoges, Éditions Lambert-Lucas, p. 177-194.

LABORDE-MILAA, I., 2007, « Des genres médiatiques aux genres didactiques : quelles transmutations? », Le français aujourd'hui, vol. 159, $\mathrm{n}^{\circ}$ 4, pp. 47-54.

LAZARD, G., 1999, « Pour une terminologie rigoureuse : quelques principes et propositions », Mémoires de la Société linguistique de Paris, Paris, p. 111-132. 
LEE, D., 2001, « Genres, registers, text types, domains and styles: Clarifying the concepts and nevigating a path through the BNC jungle », Language Learning and Technology, vol. 5, nº 3, p. 37-72.

LEGALLOIS, D., 2006, « Présentation générale. Le texte et le problème de son et ses unités : propositions pour une déclinaison », dans L. Dominique (dir.), Unité(s) du texte, Langages, 163, Paris, Larousse, p. 3-9.

LÉGLISE, I., 2017, « Multilinguisme et hétérogénéité des pratiques langagières. Nouveaux chantiers et enjeux du Global South », Langage et société, vol. 160-161, n² 2, p. 251-266.

LITS, M., 2002, «Les avatars d'un genre protéiforme », Le français aujourd'hui, vol. 138, no. 3, p. 9-18.

LUCKMANN, T., 1992, « On the communicative adjustment of perspectives, dialogue, and communicative genres ", dans A.H. Wald (éd.), The dialogical altemative. Towards a theory of language and mind, Londres, Scandinavian University Press, p. 219-234

MACÉ, M., 2006, Le Temps de l'essai. Histoire d'un genre en France au XXe siècle, Paris, Belin, coll. «L'Extrême contemporain ».

MAGRI, V., PURNELLE, G., LEGALLOIS, D., 2016, « Mot à mot, brin par brin : les suites [Nom préposition Nom] comme indices de littérarité ? ", dans D. Mayaffre et al. (éds), Journées d'Analyse des Données Textuelles, JADT), Juin 2016, Nice, France, p. 365-376

MAINGUENEAU, D., 1998/2005, Analyser les textes de communication, Paris, Armand Colin.

MAINGUENEAU, D., 2004, « Retour sur une catégorie : le genre », dans J.-M. Adam, J.-B. Grize, M. Ali Bouacha (dir.), Textes et discours : catégories pour l'analyse, Dijon, Éditions Universitaires de Dijon, collection « Langages », p. 107-118.

MAINGUENEAU, D., 2009, « Le sermon : contraintes génériques et positionnement », Langage et société, vol. 130, no. 4, p. 37-59.

MAINGUENEAU, D., 2014, Discours et analyse du discours, Armand Colin.

MALRIEU, A., RASTIER, F., 2001, « Genres et variations morphosyntaxiques », Traitements automatiques du langage, vol. 42, 2, pp. 548-577. http://www.revue-texto.net/Inedits/ Malrieu_Rastier/Malrieu-Rastier_Genres.html

MASQUELIER, B., TRIMAILLE, C., 2012, « Introduction Dell Hymes : héritages, débats, renouvellements, branchements ", Langage et société, vol. 139, n 1, p. 5-19.

MISSIRE, R., 2014, dir., Approches sémantiques de l'oral. Louvain-la-Neuve, Academia.

MOIRAND, S., 2007, « Le modèle du Cercle de Bakhtine à l'épreuve des genres de la presse », Linx, 56, p. 91-108.

MONTE, M., PHILIPPE, G. (dir.), 2014, Genres et textes : Déterminations, évolutions, confrontations, Presses universitaires de Lyon, collection « Texte \& Langue ».

MOUNIN, G., 1974, Dictionnaire de la linguistique, Paris, PUF.

MOURIQUAND, J., 2015, « La gamme des genres journalistiques », dans J. Mouriquand (éd.), L'écriture journalistique, Presses Universitaires de France, p. 58-82.

NAUDILLON, F., 2009, « Soleil, sexe et vidéo : la comédie populaire aux Antilles », Présence Francophone, revue internationale de langue et de littérature, vol. $72, \mathrm{n}^{\circ} 1$.

NAUDILLON, F., 2010, « Le théâtre populaire de José Jernidier », Africultures, vol. 80-81, n 1, p. 108-118. 
NEVEU, F., 2008, « Pour une description terminographique des sciences du langage », dans J. Humbley (éd), Cahier du CIEL 2007-2008, Aspects de la recherche en langues de spécialité, Université Paris VII, UFR EILA, p. 87-104.

NEVEU, F., 2011, Dictionnaire des sciences du langage, Paris, Armand Colin.

OESTERREICHER, W., 1979, Sprachtheorie und Theorie der Sprachwissenschaft, Heidelberg, Winter.

OESTERREICHER, W., 1993, «"Verschriftung" und "Verschriftlichung" im Kontext medialer und konzeptioneller Schriftlichkeit ", dans U. Schaefer (éd.), Schriftlichkeit imfru Men Mittelalter, Tübingen, Narr, p. 267-292.

OLLINGER, S., VALETTE, M., 2010, « La créativité lexicale : des pratiques sociales aux textes », dans Actes del I Congrés Internacional de Neologia de les llengües romaniques, CINEO'08), Barcelona, 0710 maig 2008), dans M. Teresa Cabré et al (éds), Publicacions de l'Institut Universitari de Lingüística Aplicada, IULA) de la Universitat Pompeu Fabra, UPF), p. 965-876.

PALDACCI, M., 2003, « Les quatre mondes du journal intime en ligne. Analyse statistique d'un corpus de journaux intimes écrits et publiés sur Internet », Terrains \& travaux, vol. 5, n² 2, p. 7-30. PETITJEAN, A., 1989, « Les typologies textuelles », Pratiques, 62, p. 86-125.

PORTILLO, V., 2010, « La notion de genre en Sciences du Langage », [En ligne], Volume XV, $\mathrm{n}^{\circ} 2$, 2010), URL : http://www.revue-texto.net/index.php?id=2577.

RASTIER, F., 1987, Sémantique interprétative, Paris, PUF.

RASTIER, F., 1989, Sens et textualité, Larousse.

RASTIER, F., 1999, « De la signification au sens. Pour une sémiotique sans ontologie », Texto! Textes et cultures, vol VIII, $n^{\circ} 2-3$. Disponible en ligne : http://www.revue-texto.net/index.php? $\mathrm{id}=560$

RASTIER, F., 2001a, Arts et sciences du texte, PUF, Paris.

RASTIER, F., 2001b, « Sémiotique et sciences de la culture », Linx, 44, p. 149-168.

RASTIER, F., 2007, « Conditions d'une linguistique des normes », dans G. Siouffi, A. Steuckardt, Les linguistes et la norme. Aspects normatifs du discours linguistique, Bern, Peter Lang, p. 3-20.

RASTIER, F., 2006, «Formes sémantiques et textualité », Langages, 163, p. 99-114.

RASTIER, F., 2011, « Du texte à l'œuvre : la valeur en question », dans C. Chollier (dir.), Qu'est-ce qui fait la valeur des textes?, Reims, Éditions et Presses Universitaires de Reims, p. 11-74.

RÉZEAU, P., 2018, Les mots des poilus dans leurs correspondances et leurs carnets, Éditions de linguistique et de philologie, Strasbourg.

RINGOOT, R., ROCHARD, Y., 2005, « Proximité éditoriale : normes et usages des genres journalistiques », Mots, $\mathrm{n}^{\circ}$ 77, mis en ligne le 31 janvier 2008, consulté le 30 mars 2019.

ROULON-DOKO, P., 2013, « Les genres féminins chez les Gbaya de Centrafrique », dans D. Béatrice, A. Fouque, M. Calle-Gruber (éds), Le dictionnaire universel des créatrices, Parisn, des femmes, p. 835-836.

SALES CORDEIRO, G., VRYDAGHS, D., 2016, Statuts des genres en didactique du français, Presses universitaires de Namur.

SCHNEDECKER, C., 2014, "Chaînes de référence et variations selon le genre », Langages 195, p. 23-42.

SCHAEFFER, J.-M., 1989, Qu'est-ce qu'un genre littéraire?, Paris, Seuil. 
SHERZER, J., 2012, « Langage et culture : une approche centrée sur le discours », Langage et société, vol. 139, $\mathrm{n}^{\circ} 1$, p. 21-45.

SÖLL, L., 1974/1985, Gesprochenes und geschriebenes Französisch, Berlin, Erich Schmidt Verlag.

SMITH, É., 2004, « Les cousinages de plaisanterie en Afrique de l'Ouest, entre particularismes et universalismes », Raisons politiques, vol. $\mathrm{n}^{\circ} 13$, p. 157-169.

TODOROV, T., 1970, Introduction à la littérature fantastique, Paris, Seuil.

TOMACHEVSKI, B., 1965, « Thématique », dans T. Todorov (éd.), Théorie de la littérature, Paris, Seuil, p. 273-274.

VERNIER, D., 2005, « Le chroniqueur judiciaire, observateur pertinent des tribunaux ? ", Droit et société, vol. 61, n³, p. 741-761.

VORGER, C., 2011, Poétique du slam : de la scène à l'école. Néologie, néostyle et créativité lexicale, Thèse de doctorat, Université de Grenoble.

WACH, J., 1984, Das Verstehen. Grundzüge einer Geschichte der hermeneutischen Theorie im 19. Jahrhundert, Georg Olms Verlag, Hildesheim/Zürich/ New York.

WEIDENBUSCH, W., 2006, « Historische Textsortenlinguistik: Theorie und Aufgabenbereiche / Linguistique historique des genres textuels : théorie et champs de recherche ", dans M.-D. Glessgen, C. Schmitt, W. Schweickart (éds), Sprachgeschichte / Histoire linguistique de la Romania, Berlin-New York, de Gruyter, p. 2278-2282.

WERLICH, E., 1975, Typologie der Texte, Heidelberg, Quelle \& Meyer.

WILHELM, R., 2001, « Diskurstraditionen », dans M. Haspelmath, E. König, W. Oesterreicher, W. Raible (éds), Language Typology and Language Universals. An International Handbook, I, Berlin-New York, de Gruyter, p. 467-477.

WILHELM, R., 2003, « Von der Geschichte der Sprachen zur Geschichte der Diskurstraditionen. Für eine linguistisch fundierte Kommunikationsgeschichte ", dans H. Aschenberg, R. Wilhelm (éds), Romanische Sprachgeschichte und Diskurstradition, Tübingen, p. 221-236.

WÜEST, J., 2009, « La notion de diamésie est-elle nécessaire ? », Travaux de linguistique, vol. 59, $\mathrm{n}^{\circ} 2$, p. 147-162.

ZIEGLER, A. (éd), 2010, Historische Textgrammatik und historische syntaxe des deutschen. Traditionen, innivationen, perspektive, vol. 1 et 2, Berlin-New York, de Gruyter.

ZUMTHOR, P., 1963, Langue et techniques poétiques à l'époque romane, Paris, Klincksick.

\section{NOTES}

1. J'ai plaisir à remercier les relectures vigilantes de Jean-Michel Adam, Vincent Balnat, Martin Glessgen, Julie Glikman, Mustapha Krazem et François Rastier.

2. « Nul phénomène nouveau (qu'il soit phonétique, lexical, grammatical) ne peut entrer dans le système de la langue sans être longuement passé par la mise à l'épreuve et par la finition du style-genre. » (Bakhtine 1984 : 271). En germanistique et en romanistique, cette idée correspond au concept d'« élaboration de la langue » (Kloss 1978), repris par Kabatek (2006) et formalisé dans Koch (2015a).

3. Sur le rôle de la notion de genre en anthropologie linguistique, voir en particulier Bauman (1999 et 2002). 
4. «La langue maternelle - la composition de son lexique et sa structure grammaticale - nous ne l'apprenons pas dans les dictionnaires et les grammaires, nous l'acquérons à travers des énoncés concrets [...]. Apprendre à parler c'est apprendre à structurer des énoncés [...]. Les genres du discours organisent notre parole de la même façon que l'organisent les formes grammaticales (syntaxiques). » (Bakhtine 1984 : 285).

5. Comme indice de cette marginalité, on peut noter que l'édition revue et augmentée du Dictionnaire des sciences du langage (Neveu 2011) n'octroie aucune entrée à la notion de genre (discursif).

6. Alors que Rastier appelle à «fédérer les sciences de culture» (2001b: §71) et au «remembrement des sciences du langage» (Rastier 1999), Adam (2010: 25) questionne lui l'unification des théories du texte : « les recherches locales sur des langues particulières (travaux sur les connecteurs, les anaphores, les temps verbaux, les cadratifs et autres formes de la modalisation autonymique, la position des adjectifs, les constructions détachées, etc.) peuventelles être intégrées dans un modèle général de la textualité ? Comment mettre ensemble l'abondante production relative au transphrastique, dans des langues différentes de surcroît, et les recherches qui théorisent la textualité générale?».

7. " "La" linguistique s'est diversifiée au point que cohabitent des paradigmes différents et des savoirs de plus en plus spécialisés mais, de ce fait, de plus en plus difficilement communicables. Si les linguistes travaillent, beaucoup et souvent bien, leurs recherches et leurs sociolectes se sont babélisés. " (Adam 2005: 110). De fait, les «linguistes n'ont pas organisé l'entreprise qui consisterait à s'entendre sur un minimum de connaissances admises. [...]. En l'absence de cumul des connaissances, la linguistique française semble actuellement fragile.» (BlancheBenveniste $2005: 89$ ).

8. «Existe-t-il de nos jours chez les linguistes une définition générale de l'objet de leur discipline - c'est-à-dire une définition minimale, suffisamment précise, et propre à se soutenir, ne serait-ce que de facto, d'un large consensus? [...] La réponse apparaît si incertaine qu'on pourrait se demander si la linguistique (ou les sciences du langage) n'est pas (ne sont pas), en tant que domaine unifié de recherche et d'enseignement, un paradigme plus institutionnel que scientifique. » (Bouquet $2004: 3$ ).

9. Avec Coseriu, nous postulons que «[...] toute conception et toute thèse formulée se fondent toujours sur une intuition juste, qui contient son noyau de vérité, en dépit des possibles déviations et partialisations qui peuvent survenir lors de l'explicitation de cette intuition.» (Coseriu $2004: 19$ ).

10. Ce criant déficit méthodologique n'est pas l'apanage des travaux portant sur (ou faisant simplement appel à) la notion de genre. Ainsi, Dal (2003:2) constate que les études portant sur la " productivité » lexicale ne sont pas rares à omettre d'expliciter leur cadre définitionnel.

11. Ainsi, «[...] la définition non seulement du concept de "genre" mais encore d'un concept propre à rendre compte d'une détermination supra-segmentale du sens d'un texte (ou d'un discours) ne fait pas l'unanimité parmi les chercheurs qui en explorent les pistes» (Bouquet 2004: 7). Plus généralement, pour compliquer encore la situation, "Registre», "genre", «type de texte» et même «domaine» sont des termes dont la définition varie considérablement selon le cadre théorique et les traditions scientifiques (Lee 2001). Sans parler des terminologies personnelles (ex. le «genre méronyme» de Krazem (2011a) synonyme du «genre inclus » de Rastier, etc.).

12. Par exemple : « loin d'être des "classes" (et par là, loin d'être des "genres" au sens propre du terme), ce sont bien plutôt des individus historiques, exactement comme les langues. En toute rigueur, il est impossible de définir le roman ou la tragédie en tant que classes ». (Coseriu 1971, trad. dans Gérard 2019). Voir aussi la mise au point de Schaeffer (1989: 71-74) à propos de la « classe » au sens biologique et causal. 
13. "Les débats se proposant de définir les genres de discours ont été nombreux, mais on ne s'attarde pas suffisamment sur la définition du sous-genre, comme si définir ce qui est présenté généralement comme un étage inférieur découle bien naturellement de la définition de la catégorie qui chapeaute. Or, la définition des sous-genres est une entreprise bien plus ardue qu'il n'y paraît. ». (Krazem 2011b : 49).

14. Simon Bouquet $(2004,2007)$ semble un des seuls à l'employer de manière consistante. En Allemagne, la dénomination équivalente Textsortenlinguitisk est la plus partagée, mais elle exclut habituellement de son objet les Gattungen ou genres littéraires ; une exclusion dommageable tant la réflexion sur ces derniers s'avère source d'enseignements pour la théorie des genres en général (Schaeffer 1989).

15. "le génie propre de l'auteur des "Genres du discours" est précisément, selon nous, d'opposer cette linguistique des genres à la linguistique de la langue présentée dans le Cours de linguistique générale. Prenant ainsi parti contre une linguistique de "la langue en elle-même et pour ellemême" au nom d'une linguistique réunifiée de la langue et de la parole » (Bouquet/Grillo 2007 : $\S 16)$.

16. Ou pour être plus exact, terminologiquement parlant, un espace composé de normes et de règles: "Certaines règles sont impératives, d'autres conditionnelles; mieux vaudrait donc reconnaître entre les normes des degrés d'impérativité, quitte à réserver le nom de règles aux plus impératives d'entre elles. » (Rastier $2007: 3$ ).

17. Pour un exposé, en français, sur l'importance de cette notion pour la recherche en linguistique, voir Kabatek 2015a : «Il convient de poser le concept de tradition discursive en tant que concept fondamental de la théorie linguistique, avec tout son potentiel descriptif, avant l'étude de tout phénomène concret. On doit donc le considérer comme un axiome théorique auquel tous les phénomènes concrets sont connectés a posteriori.» (p. 198-199).

18. Coseriu n'affirmait pas simplement que «l'objet de la linguistique (science du langage) ne peut être que le langage étudié sous tous ses aspects » (Coseriu $2001: 34$ ), il proposait un modèle permettant de distinguer ces différents aspects. Ainsi, « Étant donné que le langage constitue une activité humaine universelle qui se réalise individuellement mais toujours conformément à des techniques ancrées au niveau historique ('langues'), Coseriu distingue, respectivement, les trois niveaux universel, historique et individuel de la structure générale du langage. » (Koch 2015b : 78-79. Voir aussi Coseriu 2001 : 34-35). Le niveau universel correspond aux règles de l'activité de parler qui sont indépendantes des langues (voir les sept principes de la textualité : Beaugrande/ Dressler 1981 et Adamzik 2018c), le niveau individuel (ou « actuel») renvoie à l'acte d'expression dont résultent les textes concrets (appelés «discours" chez Coseriu et Koch) et le niveau historique correspond ici aux formes et règles/normes de tout idiome (langue/dialecte).

19. Nous avons proposé de compléter ce modèle en y introduisant le niveau individuel du langage (Gérard 2010 et 2018), pour situer les problématiques de l'idiolecte et du style (individuel).

20. Dont participe aussi, parmi les facteurs non-linguistiques, le statut hiérarchique des locuteurs, qui compte parmi les nombreux réglants communicationnels, comme l'illustrent la succion désapprobatrice du tchip (caribéen et africain), qu'un enfant ne peut adresser à son ainé ou à son employeur, ou l'usage du keigo (langage de politesse) au Japon.

21. Par exemple, Wilhelm (2003: 230) considère que l'inversion de la suite linguistique io et te (sur le modèle de $d u$ und ich ou toi et moi) viendrait contrarier une règle pragmatique de discours (de politesse) et que, par suite, l'ordre de ces unités ne relève pas d'une norme de langue. Au plan sémantique, les topoï et les motifs traditionnels de la folkloristique sont également des formes discursives (Rastier 2001a : 194-196), tout comme d'autres formules stéréotypées (Qui vole un cuf vole un bœuf, Il est interdit d'interdire, [Det + Nom] est dans le pré, etc.).

22. Dont relèvent aussi les styles d'époques (Biber/Conrad 2009).

23. Au Moyen-Âge, les trois registres élevé, médiocre et simple seront respectivement assimilés aux trois parties de l'œuvre de Virgile : l'Énéide, les Géorgiques et les Bucoliques. D'où la fameuse 
rota Virgilii, divisée en métiers, exemples, animaux, outils, paysages et arbres, où chacun des registres définit des prescriptions d'ordre thématique.

24. Un style fortement prescripteur de création lexicale: Brunot y voit «une richesse extraordinaire où pullulent, entassés à plaisir, mots réalistes et bas, archaïsmes et néologismes » (Brunot, Histoire de la langue française, tome II : 75).

25. On explique ainsi que la formule «Il était une fois... » soit typique du conte populaire, que le topos de «l'éloge de la patrie » soit définitoire du genre antique du propemptikon, ou encore que «dans la chanson courtoise, le motif des chants d'oiseaux comporte très souvent la série d'associations lexicales dous tens, mai ou avril, rossignol, chante cler [...]. La structure lexicale et, le plus souvent, syntaxique, reste la même » (Zumthor 1963 : 128-129).

26. Chaque ton éditorial inclut en effet, entre autres, une manière particulière de créer des mots nouveaux et de les diffuser. Cet aspect du style collectif est aujourd'hui très précisément décrit par les systèmes de détection automatique de la néologie, travaillant sur des corpus de presse (Gérard et al. 2017, Cartier 2019).

27. Depuis plus d'une dizaine d'années, l'éditorial de Libération se distingue en ce qu'il comporte un titre fait d'un seul mot, un texte non segmenté en paragraphes, un sous-emploi des mots de liaison, l'autorisation du registre familier (il s'agit d'« édito » et pas d'« éditorial »), etc.

28. Par là, les genres sont une manifestation particulière de l'altérité, une dimension spécifique du langage selon laquelle «le moi créateur de langage suppose toujours un toi auquel il s'adresse. » (Coseriu 2001 : 28-29).

29. "Aucun texte n'est écrit seulement "dans une langue" : il est écrit dans un genre et au sein d'un discours, en tenant compte évidemment des contraintes d'une langue.» (Rastier $2007: 4$ ); "[...] il n'y a pas de texte sans genre et c'est par le système de genre d'une formation sociohistorique donnée que la textualité s'articule à la discursivité » (Adam 2012 : 14).

30. Notamment, a) en général, tout genre implique certes des obligations et des interdictions, mais aussi des permissions (qualité du vocabulaire, thématique, etc.) que l'énonciateur peut négliger ou bien choisir de réaliser ; b) tous les genres ne requièrent pas une application stricte de leurs normes (comme le demande le CV japonais) ; c) le «savoir des genres » est variable et plus ou moins maîtrisé selon les individus et leur degré d'éducation (Baroni/Macé 2007).

31. Ce parallélisme entre les genres et les langues est formulé dans plusieurs publications de Coseriu, en particulier dans Coseriu 1971 (trad. dans Gérard 2019).

32. Lacoste C./Detue F. (2016, dir.) : Témoigner en littérature, revue Europe, n¹041-1042, Paris.

33. Ce genre est à rapprocher des cousinages de plaisanterie (ou parenté à plaisanterie), un genre oral bien connu des anthropologues (Smith 2004) pratiqué en Amérique du Nord, en Océanie et Afrique.

34. Le tableau suivant produit une description du genre qui conjoint les perspectives textuelle et idiomatique, qui sont rarement combinées (infra 3.2).

35. D'après Vorger (2011). D'autres normes de ce genre portent sur des aspects non-verbaux de la communication, comme l'interdiction d'utiliser des accessoires ou l'interdiction d'une parole spontanée.

36. Infra 5.1, $d$.

37. Il s'agit d'un des aspects qui distingue le slam de l'écriture rapologique : le registre vulgaire relève de l'insulte rituelle qui est le propre, notamment, du genre de la battle rap où le public apprécie la violence et le style outrageux des rappeurs en compétition.

38. Voir aussi, infra 3.1, la citation de Tomachevski.

39. Ce que Schaeffer $(1989: 67)$ résume ainsi : « [...] le système des genres théoriques, construit à partir d'oppositions différentielles, simples ou multiples, obéit à des contraintes de cohérence qui ne sont pas celles des genres historiques (quelle que soit la réalité de ces genres désignés par des noms de genres traditionnels) ». Ces "genres théoriques» correspondent notamment aux typologies de textes (Petitjean 1989) dont Gülich (1986:17) n'a pas manqué jadis de pointer le 
"déficit empirique », c'est-à-dire la distance qui les sépare de l'expérience des locuteurs participants aux situations concrètes de communication. Voir aussi à ce sujet Branca-Rosoff (2007 : § 7-13).

40. Par exemple, dans le projet Treebank Rhapsodie (www.projet-rhapsodie.fr), le talk-show mis à part, les autres "genres» décrivant le corpus sont en fait des oppositions de méthode (monologue vs dialogue, parole publique vs parole privée), sans origine ni filiation historicoculturelle. De même, Magri, Purnelle et Legallois (2016) choisissent d'organiser leur «corpus fictionnel » en opposant "roman policier » et "roman dit sérieux", cette seconde catégorie n'ayant pas d'existence socio-historique, surtout en comparaison avec la première.

41. À propos des « séquences", voir infra 3.1.

42. Boileau, dans le chant II de son Art poétique, ne fait pas qu'énumérer ces « genres mineurs » de la poésie : il rappelle leur traditionnalité en évoquant les noms des grands poètes qui les ont pratiqués (Ovide, Virgile, etc.).

43. Mais il faudrait encore ajouter aux développements précédents que la prise en compte de l'historicité des genres doit également mettre en garde contre l'usage anachronique de genres historiques pourtant bien identifiés comme tels. Par exemple, si le «Le bateau ivre " (1871) se laisse théoriquement rapprocher de l'epyllion hellénistique, ce genre ancien ne peut toutefois servir à caractériser le poème de Rimbaud, car, en cette fin de $\mathrm{XIX}^{\mathrm{e}}$ siècle, les philologues viennent à peine de le reconstruire (Cusset 2016).

44. Rastier/Malrieu (2001) : «Un champ générique est un groupe de genres qui contrastent voire rivalisent dans un champ pratique: par exemple, au sein du discours littéraire, à l'époque classique, le champ générique du théâtre se divisait en comédie et tragédie. ».

45. L'insertion de ce concept dans une représentation hiérarchique («arborescente »), comme le fait Rastier (discours > champs génériques > genres > sous-genres; infra 4.5), n'a rien de nécessaire, empiriquement parlant.

46. Entre autres: "Genres are categories established by consensus within a culture and hence subject to change as generic conventions are contested/challenged and revised, perceptibly or imperceptibly, over time." (Lee 2001: 46). Ou encore "dans le genre, l'intersubjectivité se développe en tant que condition de la subjectivité, en cela qu'il "place ce sujet en relation avec tous les sujets qui ont collaboré à l'affirmation de ce genre" et "adhère au devenir du genre qu'elle représente” (Bouquet et Grillo 2007 : §6, citant Dennes 1997).

47. Distinction reprise à Flydal (1951).

48. Voir en particulier Legallois (2006).

49. "Tout genre de discours est associé à une certaine organisation textuelle, qu'il revient à la linguistique textuelle d'étudier. Maîtriser un genre de discours, c'est avoir une conscience plus ou moins nette des modes d'enchaînement de ses constituants sur différents niveaux : de phrase à phrase mais aussi dans ses grandes parties. » (Maingueneau 1998 : 54).

50. On peut penser à Werlich (1975) ou à Beaugrande/Dressler (1981). Ces typologies, qui se laissent diviser en «classifications homogènes » et "classifications intermédiaires " (Petitjean 1989), sont contestées par Adam (1999 : 81-84) et Rastier (2001a : 257-267); voir aussi Portillo (2010 : 49) à propos de Mainguenau (2004).

51. Ainsi défini : "Genres textuels ou formes dominantes de mises en textes (proto)typées : nous pouvons entendre par là les genres narratifs, argumentatifs, explicatifs, descriptifs, dialogaux/ conversationnels, auxquels il faut ajouter des genres textuellement très contraints comme les genres de l'incitation à l'action et procéduraux [...] et les genres poétiques » (Adam 2012 : 16). Mais étant donné la remarque faite plus haut (supra 2.3), à propos de la détermination des séquences par les genres et de la nature historique de ces derniers, ce distinguo entre "genre textuel» et "genre discursif» apporte selon nous plus de confusion que de clarté terminologique.

52. Voir Ballabriga/Mpondo-Dicka (2007). 
53. Dont il faut néanmoins souligner la diversité interne (divergences d'intention théorique, variations de statut et de contenu du cercle herméneutique, etc.) : e.g. Wach (1984), Ebeling (1959), Grondin (2005).

54. "The two terms genre and register are the most confusing, and are often used interchangeably, mainly because they overlap to some degree. » (Lee $2001: 41$ ).

55. La notion de registre est définie comme « a variety associated with a particular situation of use (including particular communicative purposes)» (Biber/Conrad 2009 : 8).

56. Par exemple, la présence d'un arbre, ou analogue, pour le genre de la palabre.

57. Webster H. (1952) : La magie dans les sociétés primitives, Paris, Payot.

58. Voir par exemple la répartition thématique du forum Auféminin (forum.aufeminin.com; consulté le 18/02/2019).

59. "Whereas, in the Chinese context, to " ornate" one's argumentation academic thesis or speech with quotes of proverbial saying is highly valued and appreciated as a sign of good education, in the Western context books on style advise against using routine forms and proverbs [...]». (ibid. : 17).

60. Comme le fait, dans ce volume, Maechling au sujet de la pièce en un acte (One-Act play »), genre produit au tournant du $20^{\mathrm{e}}$ siècle, aux États-Unis.

61. Discuté et adapté au média numérique par Adamzik (2015).

62. Sur ce type de variation, infra 4.4.1.

63. On relira à cet égard les travaux pionniers de Calame-Griaule (1965). Un exemple : " "pour demander à boire en Subanum il ne suffit pas de savoir comment construire un énoncé grammatical". En effet, la traduction en Subanum de la phrase française "pourriez-vous me donner à boire ?" n'est pas comprise même si cette traduction est grammaticalement correcte. Elle n'est pas comprise parce que dans cette société la boisson est ritualisée et s'inscrit dans un code compliqué : il faut savoir à qui adresser sa demande et comment mener la joute oratoire qu'entraîne, par exemple, la boisson de bière. » (Ferry 1970 : 12).

64. La régionalisation d'un genre journalistique semble conditionnée par la «proximité éditoriale » (Ringoot/Rochard 2005).

65. Autrement dit, faible portée spatiale $=$ marquage fort ; forte portée spatiale $=$ marquage faible. 66. « le prestige linguistique est en effet lié au prestige des différents groupes sociaux, au-delà de tout élément langagier: une variété dispose d'un prestige élevé si elle est utilisée par des personnes reconnues comme importantes dans la société et vice-versa» (op. cit. : 23). Nous compléterons plus loin cette conception pour adapter la notion de prestige aux genres.

67. Ainsi nommé, au lieu de "régional ", parce que cet axe ne borne pas la notion d'espace à ce qu'on appelle couramment région (quand on parle de régionalismes pour la variation diatopique), dans la mesure où la zone d'usage d'un genre peut correspondre aux espaces plus restreints du quotidien (infra 5.4.2).

68. Cet axe est ainsi nommé car le prestige attaché à un genre est une des nombreuses représentations langagières formant l'identité culturelle d'une communauté. À ce titre, du fait de sa généralité, l'axe représentationnel pourrait comporter d'autres dimensions que le prestige culturel.

69. Genre bref humoristique : Pépin le Bref est mort depuis bientôt mille ans. / Moralité : / Quand on est mort, c'est pour longtemps. (Eugène Chavette).

70. L'invention d'un genre peut ne pas avoir de postérité et donc ne pas dépasser la phase d'innovation (ex. la stèle que son créateur, Victor Segalen, considérait explicitement comme un genre).

71. Concernant le pôle de la disparition discursive (vs innovation discursive), les exemples de genres archaïsés ne manquent pas : l'epyllion, le miracle médiéval, la criée, etc.

72. Il faudrait noter ici une phase de revitalisation entre 1970 et 1990. 
73. Blandin C. (2015) : «Le journal télévisé, incontournable ou dépassé ? », La Revue des Médias, INA, en ligne: https://larevuedesmedias.ina.fr/le-journal-televise-incontournable-ou-depasse (consulté le 18 mars 2019).

74. «Au Manding, il n'est pas permis d'énoncer les principaux genres de la culture orale n'importe où et les lieux où ils doivent être exécutés sont réglementés. Il peut même éventuellement exister des interdits «locatifs» qui excluent qu'ils soient dits en dehors d'espaces prescrits» (Derive 2009: \$3). Les espaces dont parle ici Derive sont des espaces pratiques (espace privé, espace public) et des espaces physiques (ex. les champs, la brousse, etc.), qu'il nomme « contingent».

75. «Le Bwiti (ou Bwete) est une tradition religieuse qui provient des Mitsogo, une population du sud du Gabon, mais qui s'est diffusée depuis longtemps déjà dans une large partie du pays, y compris en milieu urbain. » (Bonhomme 2018).

76. En entendant région au sens d'une « portion de territoire ou d'espace géographique » (TLFI), à laquelle correspond souvent un statut administratif dans les pays occidentaux, mais qui peut être dépourvue de définition officielle (ex. chez les peuples racines).

77. Source : «Les Français et la lecture - 2019 », A. Vincent Gérard et J. Poncet, 2019, Centre national du livre.

78. Cet exemple montre que l'observation d'une variété spatiale doit absolument tenir compte de l'histoire.

79. On se méfiera néanmoins de certains noms de genre. Par exemple, ce qu'on appelle le roman régional ne correspond pas du tout, malgré son nom, à une variété régionale : ce n'est pas parce que le roman régional de Flandre (De Geest 2006) singularise son contenu en puisant dans la toponymie, les coutumes, l'histoire locale, etc. que ce modèle communicationnel est particulièrement lié à la culture de cette région.

80. $\mathrm{Au}$ reste, comme les genres "codifient" le statut des acteurs de la communication représentée et réelle (ex. énonciateur réel/fictif/feint, destinataire déterminé/indéterminé, etc. ; Schaeffer 1989 : 82-100), la théorie des genres devrait plus explicitement intégrer le point de vue des acteurs réels de la communication, dont le statut social est loin d'être neutre concernant l'appropriation et la reconnaissance d'un genre (Baroni/Macé 2007) et, par suite, l'interprétation même du texte (Schaeffer 1989 : 129).

81. Sur le savoir linguistique des locuteurs, et en particulier leur savoir expressif (celui qui, précisément, correspond aux genres), voir Coseriu (1988), exposé en français dans Gérard (2016).

82. Certains abordent aussi cette problématique de la didactisation des genres en parlant de « genre prescrit » et de « genre construit» (Bouillon 2007).

83. En France, le twiller intitulé « 3ème droite », de François Descraques, remporte actuellement un grand succès populaire.

84. "Toute actualisation d'un genre discursif a une histoire en même temps qu'il est lui-même dans l'histoire. Ainsi l'interview dialoguée dans la presse écrite s'est développée après que les lecteurs ont intériorisé le genre à la radio : la forme qu'il prend dans la presse est cependant fort éloignée de l'entretien oral qui l'a précédé (il s'agit rarement d'une transcription et les contraintes d'espace et de lisibilité sont importantes). » (Moirand 2007 : §33).

85. Voir supra 4.1, les propositions de Günthner/Knoblauch.

86. Soit chanson paillarde $=\mathrm{a}$; annonce mortuaire (Alsace) $=\mathrm{b} ;$ acte authentique de notaire $=\mathrm{c}$; slam $=$ $\mathrm{d} ;$ conférence scientifique $=\mathrm{e} ;$ polar régional $=\mathrm{f} ;$ battle rap $=\mathrm{g} ;$ causerie $($ conversation familière $)=\mathrm{h}$.

87. On voit ici que les «textes de loi» (Koch/Oesterreicher 2001, Glessgen 2018), dont la dénomination est entre parenthèses trop vague pour correspondre à un genre historique, ne se positionnent pas automatiquement à l'extrémité de la distance communicationnelle.

88. Selon la description de l'acte authentique sur le site www.notaires.fr (consulté le 26/03/2019). 
89. Dans la communication scientifique (de réalisation phonique), on distingue traditionnellement l'intervention en séminaire de recherche, la communication en colloque et la conférence plénière.

90. Par exemple, logiquement, un entretien d'embauche (en face à face) au siège d'une multinationale (Paris) doit manifester un degré de diaphasie et de diastratie plus élevé qu'un entretien se déroulant dans une petite entreprise de province.

\section{RÉSUMÉS}

Quel est l'objet de la linguistique des genres et de quelle complexité est fait ce champ d'études? Quels critères objectifs doivent assurer le maniement de la notion de genre ? En quoi réside la complexité de la relation entre un texte et « son » genre ? Quelle est la pertinence de la notion de « sous-genre "? Quels observables centraux restent négligés par la linguistique des genres ? Pour répondre à ces questions, qui témoignent d'une discipline en crise, cet article non seulement réaffirme le lien indissoluble entre histoire, culture et genres discursifs, mais entend repenser la linguistique des genres du point de vue variationniste - comme l'appelle clairement l'étude ethnologique des phénomènes communicationnels. Dans ce but, on introduit ici le concept de variété générique et des distinctions méthodologiques permettant la mise en œuvre d'une approche variationniste des genres en linguistique.

What is the subject of genre linguistics and how complex is this field of study? Which objective criteria must ensure the handling of the notion of gender? What is the complexity of the relationship between a text and "its" genre? What is the relevance of the «sub-genre » concept? Which main topic of study remain neglected by genre linguistics? To answer these questions, which testify to a discipline in crisis, this article not only reaffirms the immutable link between history, culture and discursive genres, but also intends to rethink gender linguistics from a variational point of view - as it is clearly requested by the ethnological study of communicative phenomena. To this purpose, we introduce here the concept of generic variety and some methodological distinctions allowing the implementation of a variational approach to genres in linguistics.

\section{INDEX}

Keywords : Variation, varieties, discourse genres

Mots-clés : Variation, variété, genres discursifs, genre textuel, linguistique textuelle, sémiotique, ethnologie

\section{AUTEUR}

\section{CHRISTOPHE GÉRARD}

Université de Strasbourg 